ARRHYTHMIA ANALYSIS IN LANGENDORFF PERFUSED HEARTS: SIGNIFICANT CONTRIBUTION TO MODEL CHARACTERISATION AND VALIDATION OF ARRHYTHMIA DEFINITIONS

\begin{abstract}
Hedvig Takács, MD
Second Department of Internal Medicine and Cardiology Centre,

University of Szeged
\end{abstract}

PhD Thesis

Szeged

2019 


\section{THE THESIS IS BASED ON THE FOLLOWING PAPERS}

I. Takács, H; Kui, P; Farkas, A S; Sarusi, A; Forster, T; Papp, J Gy; Varró, A; Curtis, M J; Shattock, M J; Farkas, A; Ventricular cycle length irregularity affects the correlation between ventricular rate and coronary flow in isolated, Langendorff perfused guinea pig hearts. Journal of Pharmacological and Toxicological Methods. 77 pp. 45-52., 8 p. (2016).

Impact factor (2016): 2.238

II. Regev, A; Takacs, H; Farkas, A S; Rarosi, F; Polyak, A; Papp, H; Ivany, E; Papp, J Gy; Varro, A; Farkas, A; Application of ventricular tachyarrhythmia definitions of the updated Lambeth Conventions provides incompatibility with earlier results, masks antifibrillatory activity and reduces inter-observer agreement. Journal of Physiology and Pharmacology 70: 1 (2019).

Assaf Regev and Hedvig Takacs equally contributed and share first authorship.

Impact factor (2018): 2.544

\section{FURTHER RELEVANT PAPERS}

III. Kui, P; Orosz, S; Takács, H; Sarusi, A; Csík, N; Rárosi, F; Csekő, C; Varró, A; Papp, JG; Forster, T; Farkas AS; Farkas A; New in vitro model for proarrhythmia safety screening: IKs inhibition potentiates the QTc prolonging effect of IKr inhibitors in isolated guinea pig hearts. Journal of Pharmacological and Toxicological Methods 80 pp. 26-34., 9 p. (2016).

Impact factor (2016): 2.238

IV. Papp, H; Sarusi, A; Farkas, AS; Takacs, H; Kui, P; Vincze, D; Ivany, E; Varro, A; Papp, JG; Forster, T; Farkas A; Hyperventilation assists proarrhythmia development during delayed repolarization in clofilium-treated, anaesthetized, mechanically ventilated rabbits. Journal of Physiology and Pharmacology 67: 5 pp. 731-737. , 7 p. (2016).

Impact factor (2016): 2.883 


\section{QUOTABLE ABSTRACTS}

I. Takács, H; Farkas, AS; Forster, T; Varró, A; Farkas, A; Az új Lambeth convenciós módosított kamrafibrilláció definíciója megváltoztathatja az aritmiavizsgálatok konklúzióját. (The new definition of ventricular fibrillation of the upgraded Lambeth conventions may substantially alter the conclusions of arrhythmia investigations); Cardiologia Hungarica 44: Suppl. E pp. E38-E39. (2014)

II. Takács, H; Kui, P; Farkas, A; Forster, T; Varró, A; Farkas, A; A kamrai irregularitás okozta koronáriaáramlás-növekedés mechanizmusa izolált, langendorff perfundált tengerimalac szívben (The mechanism of increased coronary flow caused by ventricular irregularity in isolated, langendorff perfused guinea pig hearts); Cardiologia Hungarica 45: Suppl. D pp. D25D26. (2015)

III. Kui, P; Takács, H; Morvay, N; Leprán, I; Farkas, A; Varró, A; Forster, T; Farkas, A; Sportszív kamrahipertrófia modell beállítása nyulakban (Setting up an athlete's heart model in rabbits); Cardiologia Hungarica 45: Suppl. D p. D34 (2015)

IV. Prorok, J; Kui, P; Takács, H; Oravecz, K; Hézső, T; Polyák, A; Farkas, AS; Papp, JGy; Varró, A; Tóth, A; Acsai K; A szelektív NCX-gátlás csökkenti a hypokalaemia és a kamrafibrilláció által okozott miokardiális diszfunkciót. (Selective NCX inhibition reduces myocardial dysfunction associated with hypokalaemia and ventricular fibrillation); Cardiologia Hungarica 46: Suppl. F p. F60 (2016)

V. Kui, P; Takács, H; Morvay, N; Leprán, I; Tiszlavicz, L; Nagy, N; Ördög, B; Farkas, A; Forster, T; Varró, A; Farkas AS; Repolarizációs érzékenység vizsgálata nyúl sportszívmodellben. (Examination of the repolarization sensitivity in a rabbit athlete's heart model); Cardiologia Hungarica 46: Suppl. F p. F40 (2016)

VI. Kui, P; Takács, H; Morvay, N; Leprán, I; Tiszlavicz, L; Nagy, N; Ördög, B; Farkas, A; Forster, T; Varró, A; Farkas AS; Proaritmia érzékenység vizsgálata nyúl sportszív modellben (Assessment of proarrhythmia sensitivity in a rabbit athlete's heart model); Sportorvosi Szemle 57: 1 p. 7 (2016)

VII. Kui, P; Takács, H; Polyák, A; Morvay, N; Leprán, I; Nagy, N; Ördög, B; Farkas, A; Forster, T; Varró, A; Farkas AS; Investigation of electrophysiolological abnormalities in a rabbit athlete's heart model; Cardiovascular Research 111: Suppl. 1 p. S101 (2016) 


\section{SUMMARY}

In this work, we used the isolated, Langendorff perfused heart model for arrhythmia investigations, and the data of the arrhythmia analysis served for clarifying and characterising the physiology of the model and also, to validate arrhythmia definitions.

In our first investigation we examined the relationship between ventricular rhythm and coronary flow autoregulation in Langendorff perfused guinea pig hearts. It is a well-known fact, that heart rate affects coronary flow, but the mechanism is complex, especially in experimental settings. We examined whether ventricular irregularity influences coronary flow independently of heart rate. According to our results, during regular rhythm, left ventricular pressure exceeded perfusion pressure and prevented coronary perfusion at peak systole. However, ventricular irregularity significantly increased the number of beats in which left ventricular pressure remained below perfusion pressure, facilitating coronary perfusion. We found that in isolated hearts, cycle length irregularity increases the slope of the positive linear correlation between mean ventricular rate and coronary flow via producing beats in which left ventricular pressure remains below perfusion pressure. This means that changes in rhythm have the capacity to influence coronary flow independently of heart rate in isolated hearts perfused at constant pressure.

In our second investigation we examined whether the arrhythmia definitions of Lambeth Conventions I (LC I) and Lambeth Conventions II (LC II) yield the same qualitative results and whether LC II improves inter-observer agreement. Data obtained with arrhythmia definitions of LC I and LC II were compared within and between two independent observers. Applying ventricular fibrillation (VF) definition of LC II significantly increased VF incidence and reduced VF onset time irrespective of treatment by detecting 'de novo' VF episodes. Using LC II reduced the number of ventricular tachycardia (VT) episodes and simultaneously increased the number of VF episodes, and thus, LC II masked the significant antifibrillatory effects of flecainide and the high $\mathrm{K}^{+}$concentration. When VF incidence was tested, a very strong interobserver agreement was found according to LC I, whereas using VF definition of LC II reduced inter-observer agreement. It is concluded that LC II shifts some tachyarrhythmias from VT to VF class. VF definition of LC II may change the conclusion of pharmacological, physiological and pathophysiological arrhythmia investigations and may reduce inter-observer agreement.

\section{TABLE OF CONTENTS}


1. INTRODUCTION 1

1.1. Background of the first study - Understanding the relationship between ventricular rhythm and coronary flow autoregulation in Langendorff perfused hearts...

1.2. Background of the second study - Application of ventricular tachyarrhythmia definitions of the updated

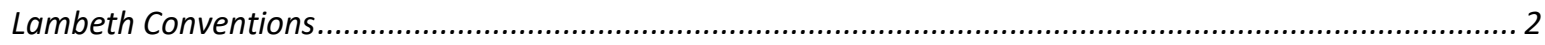

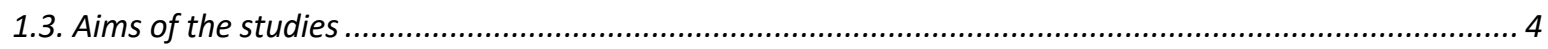

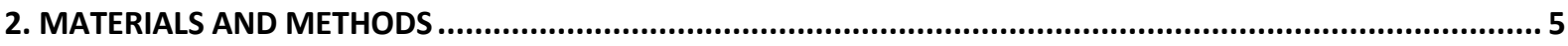

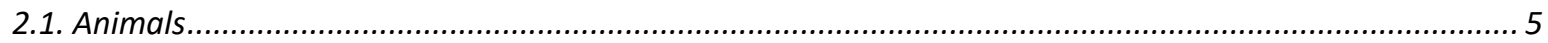

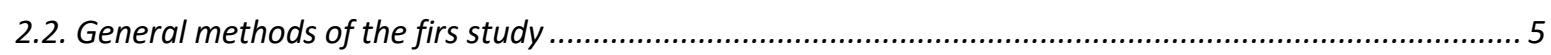

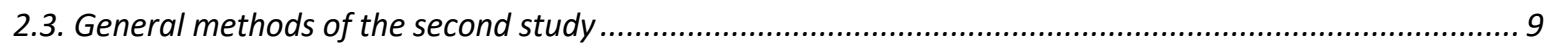

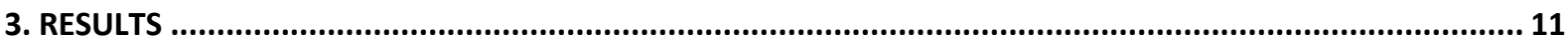

3.1. Results of the first study - Understanding the relationship between ventricular cycle length irregularity and coronary flow in Langendorff perfused guinea pig hearts .................................................................. 11

3.2. Result of the second study - Comparing arrhythmia results obtained according to the old and the updated Lambeth Conventions, validation of arrhythmia definitions...................................................... 19

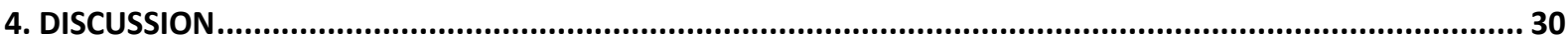

4.1. Understanding the correlation between increased beat-to-beat variability of ventricular cycle length and coronary flow in Langendorff perfused hearts .................................................................................... 30

4.2. Comparing arrhythmia results obtained according to the old and the updated Lambeth Conventions validation of arrhythmia definitions by using arrhythmia data from Langendorff perfused hearts ............... 32

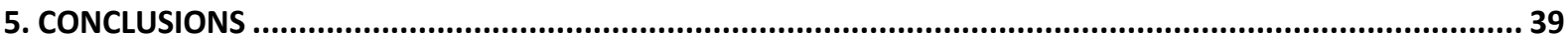

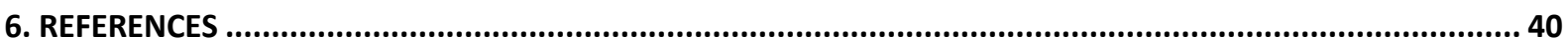

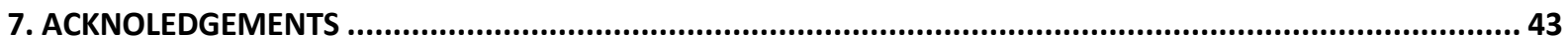




\section{INTRODUCTION}

Oscar Langendorff described the experimental, isolated, perfused heart model more than 100 years ago (1). Still, isolated, Langendorff perfused mammalian hearts are valuable tools in heart research even today, and the Langendorff heart is still one of the most frequently used experimental models in cardiovascular research (2). In spite of the progressive evolution of computer simulations, in-silico models, and cellular electrophysiological techniques in for example drug induced proarrhythmic testing (3), the Langendorff system is still a simple, costeffective and useful cardiovascular model. Despite its simplicity, the model is applicable for physiological and pathophysiological studies, and molecular biological techniques and genetic manipulation made its use even wider (2). In our investigations, we used the Langendorff perfused heart model for arrhythmia investigations, and the data of the arrhythmia analysis served for clarifying and characterising the physiology of the model and also, to validate arrhythmia definitions.

\subsection{Background of the first study - Understanding the relationship between ventricular}

\section{rhythm and coronary flow autoregulation in Langendorff perfused hearts}

Understanding the relationship between ventricular rhythm and coronary flow autoregulation in experimental preparations such as the Langendorff is important for several reasons.

In a clinical standpoint cardiac arrhythmias and their impact to the heart and its function are important questions. Many patients live with irregular ventricular rate caused by either frequent ventricular or atrial arrhythmias. Irregular ventricular rate may be harmful in the long term, e.g. it is well documented that frequent ventricular premature beats (VPB) can lead to development of cardiomyopathy (4). However, it is not known whether an effect of irregular ventricular rhythm on coronary flow may contribute to the harmful effects of irregular ventricular rhythm.

In in vivo conditions coronary flow is regulated by a combination of i) intramural pressure in coronary arteries caused by wall stress during the cardiac cycle, ii) the autonomic nervous system, iii) and work-induced autoregulation via local metabolites $(5,6)$. Additionally, irregular rhythm affects the work of the myocardium independently of rate and load (7). This implies that irregular ventricular rhythm may have an independent effect on coronary flow via modifying the work-induced autoregulation of the coronary arteries. However, this has never 
been examined, and data about the well-known positive correlation between ventricular rate and coronary flow have been obtained from hearts free of arrhythmias $(8,9)$.

\subsection{Background of the second study - Application of ventricular tachyarrhythmia} definitions of the updated Lambeth Conventions

Sudden cardiac death caused by ventricular arrhythmias is a major public health problem in modern industrialized countries (10), which emphasizes the importance of arrhythmia research. Currently, there are clinical $(10,11)$ and experimental guidelines $(12,13)$ dealing with arrhythmia research and management, but these guidelines do not use unified arrhythmia definitions, and that is a notable problem when we try to objectively evaluate and compare results of arrhythmia investigations.

The Lambeth Conventions (LC I), a guidance for research on arrhythmias published in $1988(12,13)$, had a substantial impact on the experimental arrhythmia research; the paper has been cited more than 1000 times since its publication 30 years ago according to the database of Web of Science. However, the advances in technology, development of monitoring and pharmacologic solutions and of course the extensive research about arrhythmias had finally led to the realization that LC I was in need to be updated. Thus, a meeting was held in London in 2010 to update the guidance. The revised conventions were intended to be of practical value in terms of the design, execution, and analysis of experiments, with emphasis on the definition, classification, and quantification of ventricular and atrial arrhythmias (13). The revised Lambeth Conventions (LC II) were intended to be applied in preclinical and clinical research. Authors of LC II invited investigators to state whether or not they had used the conventions in their studies, and to test their validity by experiment. Importantly, there are substantial changes in the definitions of the ventricular tachyarrhythmias between the original and the updated Lambeth Conventions (see Table 1 for the comparison of arrhythmia definitions of LC I and LC II). The new tachyarrhythmia definitions imply that some arrhythmias classified as ventricular tachycardia (VT) according to LC I are now classified as ventricular fibrillation (VF) according to LC II. This suggests that the new definitions change the results and - more importantly - the conclusion of arrhythmia studies. 
Table 1. Comparison of the definitions of ventricular arrhythmias between the Lambeth Conventions I \& II.

\begin{tabular}{|c|c|c|}
\hline Arrhythmia & $\begin{array}{c}\text { Definition according to } \\
\text { Lambeth Conventions I (12) }\end{array}$ & $\begin{array}{l}\text { Definition according to } \\
\text { Lambeth Conventions II } \\
\text { (13) }\end{array}$ \\
\hline $\begin{array}{l}\text { Ventricular premature beat } \\
\text { (VPB) }\end{array}$ & $\begin{array}{l}\text { Isolated ventricular premature } \\
\text { beats are defined as discrete and } \\
\text { identifiable premature QRS } \\
\text { complexes (premature in } \\
\text { relation to the P wave). }\end{array}$ & $\begin{array}{l}\text { A VPB is defined as a } \\
\text { ventricular electrical complex } \\
\text { (complete electrical event: QRS, } \\
\text { RS, QRST or RST) that is } \\
\text { different in shape (voltage } \\
\text { and/or duration, i.e., height } \\
\text { and/or width) from the } \\
\text { preceding (non-VPB) } \\
\text { ventricular complex, and is } \\
\text { premature in relation to the } \\
\text { preceding ventricular complex. }\end{array}$ \\
\hline Bigeminy & $\begin{array}{l}\text { Bigeminy is characterised by the } \\
\text { minimum sequence: } P, Q R S \text {, } \\
\text { VPB, P, QRS. VPB. }\end{array}$ & $\begin{array}{l}\text { Bigeminy has the minimum } \\
\text { sequence VPB, normal sinus } \\
\text { beat, VPB (which may be } \\
\text { repeated) in which the VPBs } \\
\text { have the same shape and timing. }\end{array}$ \\
\hline Salvo & $\begin{array}{l}\text { Two or three consecutive VPBs } \\
\text { do not constitute ventricular } \\
\text { tachycardia but should be } \\
\text { termed a salvo. }\end{array}$ & $\begin{array}{l}\text { A run of two or three } \\
\text { consecutive VPBs is defined as } \\
\text { a salvo. }\end{array}$ \\
\hline Ventricular tachycardia (VT) & $\begin{array}{l}\text { A run of } 4 \text { or more consecutive } \\
\text { ventricular premature beats. }\end{array}$ & $\begin{array}{l}\text { A sequence of a minimum of } 4 \\
\text { consecutive ventricular } \\
\text { complexes. } \\
\text { Monomorphic VT: } \\
\text { peak-peak interval, height and } \\
\text { intrinsic shape are constant. } \\
\text { Polymorphic VT: } \\
\text { the peak-peak interval and/or } \\
\text { height and/or intrinsic shape } \\
\text { vary, and the variation of any } \\
\text { or each of these is progressive. }\end{array}$ \\
\hline Ventricular fibrillation (VF) & $\begin{array}{l}\text { A signal for which individual } \\
\text { QRS deflections can no longer } \\
\text { be distinguished from one } \\
\text { another (implying } \\
\text { morphological instability) and } \\
\text { for which a rate can no longer be } \\
\text { measured. }\end{array}$ & $\begin{array}{l}\text { A sequence of a minimum of } 4 \\
\text { consecutive ventricular } \\
\text { complexes without intervening } \\
\text { diastolic pauses, in which } \\
\text { intrinsic shape, peak-peak } \\
\text { interval and height vary, and the } \\
\text { variation between each is non- } \\
\text { progressive. It is the non- } \\
\text { progressive nature of the } \\
\text { variation of all } 3 \text { variables that } \\
\text { distinguishes VF from } \\
\text { polymorphic VT and torsades de } \\
\text { pointes. }\end{array}$ \\
\hline
\end{tabular}




\subsection{Aims of the studies}

As it is not known how work-induced autoregulation of coronary arteries is affected by ventricular irregularity, the approach of the first study was to determine the effect of beat-tobeat variability of ventricular cycle length on coronary flow in isolated, Langendorff-perfused guinea pig hearts. Caval veins do not fill the right atrium in Langendorff-perfused heart, and thus when the hydrostatic pressure of the perfusion column is constant, hearts can be studied with coronary arteries perfused under constant pressure (14). Also, Langendorff-perfused hearts are normally denervated. Thus, under these conditions coronary flow is regulated independently of perfusion pressure and autonomic nervous system, and only intramural pressure during the cardiac cycle and work-dependent autoregulation determine coronary resistance. As ventricular irregularity was found to significantly affect coronary flow in the present investigation, the mechanism was examined in a further set of experiments performed in Langendorff perfused guinea pig hearts.

In the second study the aim was to examine whether the arrhythmia definitions of LC I and LC II are compatible, and yield the same qualitative arrhythmia results. Also, it was tested whether arrhythmia definitions of LC I or LC II allow better inter-observer agreement. Thus, two independent investigators reanalysed the electrocardiogram (ECG) recordings of experiments done earlier by Farkas and Curtis (15), and performed a retrospective, blinded analysis of the number and incidence of ventricular arrhythmias in isolated, Langendorff perfused rat hearts subjected to regional ischemia and treated with Class I antiarrhythmic agents. The arrhythmia data obtained by applying arrhythmia definitions of LC I and LC II were compared to test the compatibility between the arrhythmia definitions of LC I and LC II. Also, inter-observer agreement was determined to investigate whether arrhythmia definitions of LC I or LC II allow better agreement on the arrhythmia results between the two independent observers. 


\section{MATERIALS AND METHODS}

\subsection{Animals}

In the first study female guinea pigs ( $n=87$ in the first set of experiments and $n=33$ in the second), weighing 300-400 g were used. The animal-handling protocol was in accordance with the Guidance of the Operation on the Animals (Scientific Procedures) Act 1986 and the European Community guidelines for the use of experimental animals.

In the second study we reanalysed the ECG recordings of a previous investigation utilizing isolated, Langendorff perfused rat hearts. For detailed methodical description see the published study by Farkas and Curtis (15), from which the raw ECGs were obtained for arrhythmia analysis. For this previous study $n=144$ male rat hearts were used, the animal-handling protocol was in accordance with the Guidance of the Operation on the Animals (Scientific Procedures) Act 1986, London, UK.

\subsection{General methods of the firs study}

\subsubsection{Details of Langendorff perfusion}

The method of Langendorff perfusion we used has been described in detail (15-17). Briefly, guinea pigs were anesthetized with pentobarbital $(60 \mathrm{mg} / \mathrm{kg}$ i.p.) mixed with $1000 \mathrm{IU}$ sodium heparin to prevent blood clot formation in the coronary vasculature. Sodium heparin (500 IU) was additionally administered i.v. Hearts were excised and placed immediately into ice-cold modified Krebs-solution containing: $118.5 \mathrm{mM} \mathrm{NaCl}, 25.0 \mathrm{mM} \mathrm{NaHCO} 3,0.5 \mathrm{mM}$ $\mathrm{MgSO}_{4}, 1.2 \mathrm{mM} \mathrm{NaH} 2 \mathrm{PO}_{4}, 1.8 \mathrm{mM} \mathrm{CaCl}, 3.0 \mathrm{mM} \mathrm{KCl}, 11.1 \mathrm{mM}$ glucose. Langendorff perfusion was begun with solution delivered at $37^{\circ} \mathrm{C}$ and $\mathrm{pH} 7.4$ at constant perfusion pressure (60 $\mathrm{mmHg})$. In the first set of experiments, a unipolar electrogram (ECG) was recorded by implanting one stainless-steel wire electrode into the middle of the anterior wall of the left ventricle with a second connected to the aorta. In the second set of experiments, volume conducted ECG was recorded by submerging the ventricles of the hearts in modified Krebs solution at $37^{\circ} \mathrm{C}$.

\subsubsection{Measurement of coronary flow}

Coronary flow was measured by timed collection of coronary effluent. At the end of the experiment atria were removed from the hearts and ventricles were weighed. Coronary flow values are shown in $\mathrm{ml} / \mathrm{min} / \mathrm{g}$. 


\subsubsection{ECG analysis, measurement of the RR intervals and calculation of variability of the} ventricular cycle length in the first set of experiments

The ECG was recorded and analysed by LabChart7 (ADInstruments Ltd, Oxford, UK). In the guinea pig Langendorff preparation, non-complex arrhythmias (mostly VPBs, atrial premature beats $[\mathrm{APBs}]$, and sinus arrhythmia) occur frequently during the initial period after mounting the heart, especially if Krebs solution contains a low concentration of $\mathrm{K}^{+}(3.0 \mathrm{mM})$ and a high concentration of $\mathrm{Ca}^{2+}(1.8 \mathrm{mM})$. However, these arrhythmias spontaneously resolve within 20-30 min. The reason for these baseline arrhythmias is not known; it is a particular feature of guinea pig hearts and the same perfusion method does not cause arrhythmias in rat and rabbit hearts $(15,18)$. These ventricular and atrial arrhythmic beats were defined according to the Lambeth Conventions II (13) and manually counted in the last 30 seconds of the 30minute-long control perfusion period. The per cent frequency of arrhythmic beats (defined as APBs, VPBs or individual QRT complexes in a run of a salvo or tachycardia) was calculated in the sampling period as the number of ventricular arrhythmic beats divided by the total number of beats times 100 .

The RR intervals were measured irrespective of rhythm even during arrhythmias in the 30 -second-long sampling period. The mean ventricular rate was calculated as the total number of ventricular complexes (arrhythmic or not) times 2 . The beat-to-beat variability of the RR intervals was quantified by the root mean square of the successive differences of the RR intervals (RMSSD) as described previously (19): taking the successive differences of the RR intervals $\left(\Delta d_{j}=d_{j+1}-d_{j} ; 0 \leq j \leq N-2\right.$, where $d_{j}$ represents the RR or QT interval durations and $N$ is the total number of intervals) and calculating $\operatorname{RMSSD}=\sqrt{E\left([\Delta d]^{2}\right)}$, where $E$ denotes the mean value. RMSSD of the RR interval during sinus rhythm in vivo is a parameter widely used for quantifying heart rate variability as a biomarker of parasympathetic activity $(20,21)$, but in the Langendorff preparation it quantifies only the irregularity of the cycle length since parasympathetic tone is absent.

These hearts were divided into two groups based on RMSSD value as the 'Low' RR variability group (RMSSD $<3 \mathrm{~ms}$; $\mathrm{n}=50$ hearts), and the 'High' $\mathrm{RR}$ variability group (RMSSD $>3 \mathrm{~ms} ; \mathrm{n}=37$ hearts) (Figure 1.). The beat-to-beat variability of the cycle length (RMSSD), the per cent frequency of the arrhythmic beats, the mean ventricular rate and the coronary flow were compared between the 'Low' and 'High' RR variability groups. Note (with respect to justification of animal usage) that all hearts were entered into a separate, unrelated experimental protocol $30 \mathrm{~min}$ after the start of perfusion. 


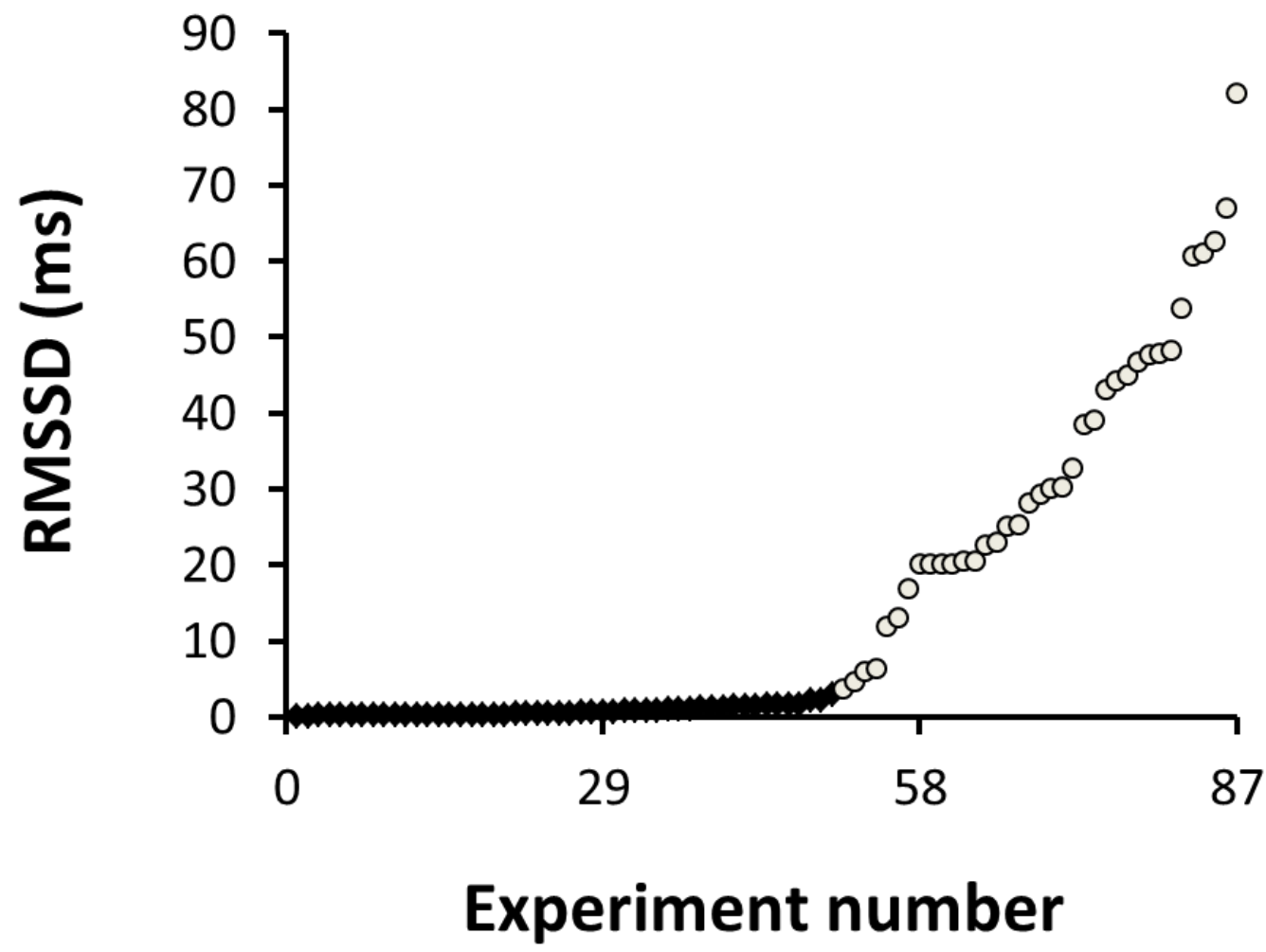

Figure 1. The beat-to-beat variability of the ventricular cycle length quantified by the root mean square of the successive differences of the RR intervals (RMSSD) in 87 Langendorff perfused guinea pig hearts in the first set of experiments. Experiments are sorted by increasing order of the RMSSD. The 87 hearts were retrospectively divided into two groups based on the value of the RMSSD parameter. That is, a 'Low' RR variability group (RMSSD $<3 \mathrm{~ms}$; $\mathrm{n}=50$ hearts, dark squares), and a 'High' RR variability group (RMSSD $>3 \mathrm{~ms}$; $n=37$ hearts, light circles) were arbitrarily defined.

\subsubsection{Measurement of the duration of perfused and non-perfused intervals in every cardiac}

cycle

It was found in the first set of experiments that cycle length irregularity increased the slope of the linear correlation between mean ventricular rate and coronary flow. Myocardium in vivo is perfused during diastole and is not perfused when intramural capillaries are compressed by intramural pressure during systole. In order to examine whether elevated RR interval variability increased the ratio of durations of perfused intervals to non-perfused intervals in the Langendorff preparation, a second set of experiments was performed with 33 hearts perfused identically for 60 minutes with the same modified Krebs solution but with hearts submerged in Krebs solution in order to record volume conducted ECG. Left ventricular pressure was recorded via a thin medical needle stuck through the apex of the heart, and attached to a plastic cannula filled with saline. Perfusion pressure (aortic pressure) was recorded 
via a side arm at the bottom of the perfusion column where perfusion cannula was connected to the aorta. "Real-time" aortic flow was measured by an ultrasonic flow meter (T106 Animal Research Flowmeter, Transonic Systems Inc. Ithaca, NY, U.S.A) implanted to the bottom of the perfusion column, closely above the aortic stump. Krebs solution was continuously pumped to the reservoir by a pump (Peri-star Pro, World Precision Instruments, Sarasota, Florida, U.S.A.) during the experiment and a built-in overflow system kept the height of the column at a pre-set, constant level, thus the hydrostatic pressure of the perfusion column was constant (65 $\mathrm{mmHg}$ ). Volume-conducted ECG, real-time aortic flow, aortic and left ventricular pressures were recorded continuously by using National Instruments data acquisition hardware (PC card, National Instruments, Austin, TX., U.S.A.) and SPEL Advanced Haemosys software (version 3.26, Experimetria Ltd. and Logirex Software Laboratory, Budapest, Hungary).

RR interval variability (RMSSD of the RR interval), mean ventricular rate and mean aortic flow were determined in every 30-second-long interval during the 60-minute-long perfusion period in each heart. A strong, positive, linear correlation was found between the measured coronary flow and the calculated mean aortic flow values $(y=1.0166 x+0.5482$ where $\mathrm{y}$ is the mean aortic flow value $(\mathrm{ml} / \mathrm{min} / \mathrm{g})$ and $\mathrm{x}$ is the measured coronary flow value $(\mathrm{ml} / \mathrm{min} / \mathrm{g})$; regression coefficient: $\mathrm{R}=0.966 ; \mathrm{P}<0.0001$, linear regression). In 15 hearts, the ventricular rate did not match the mean ventricular rate found in the first set of experiments, thus these hearts were excluded from the analysis. In each of the remaining 18 hearts, there was at least one 30-second-long interval in which mean ventricular rate matched the mean ventricular rate measured in the first set of experiments. One of these 30-second-long intervals was chosen for further analysis in each heart. These 18 hearts thus replicated the RR interval variability and mean ventricular rate of the 'Low' and 'High' RR variability groups in the first set of experiments, with 9 hearts allocated into a 'Low' RR interval variability group (RMSSD $<3 \mathrm{~ms}$ ), and the other 9 to a 'High' RR interval variability group (RMSSD $>3 \mathrm{~ms}$ ).

An analysis of the durations of perfused and non-perfused intervals in the 18 hearts was performed in a blinded manner. The number of beats in which left ventricular pressure did not exceed perfusion pressure during systole, and the duration of the non-perfused intervals during each systole were determined. The non-perfused interval (i.e. the interval where intramural pressure prevents coronary perfusion) was defined as the interval in which left ventricular pressure was greater than perfusion pressure during systole. Perfused interval (i.e. the interval where intramural pressure allows coronary perfusion) was defined as the interval in which left ventricular pressure was lower than perfusion pressure. The ratio of the perfused to nonperfused intervals was calculated by dividing the cumulative duration of the perfused intervals 
by the cumulative duration of the non-perfused intervals in the 30 -second-long sampling interval at the time point of the measurement.

\subsubsection{Statistical evaluation of the results}

Continuous data were expressed as mean \pm standard deviation (SD) and the 'Low' and 'High' RR variability groups were compared using the non-parametric Mann-Whitney U test. Within group comparison of continuous data was performed using the non-parametric Wilcoxon test. The coronary flow values were plotted against the mean ventricular rate values. The correlation between mean ventricular rate and coronary flow was examined with analysis of covariance, in which variables of coronary flow (as the continuous dependent variable), mean ventricular rate (as the continuous predictor variable, i.e. covariate) and group ('Low' versus 'High' RR variability group, as the categorical grouping variable) were tested. $\mathrm{P}<0.05$ was taken as indicative of a statistically significant difference between values.

\subsection{General methods of the second study}

\subsubsection{Description of the Langendorff perfusion}

For detailed methodical description see the published study by Farkas and Curtis (16), from which the raw ECGs were obtained for arrhythmia analysis. Thus, here only a short description of the experiments is added as follows. Male rats were anesthetized with pentobarbitone (60 mg/kg intraperitoneally). Hearts were excised, and then perfused according to Langendorff with modified Krebs solution containing $118.5 \mathrm{mM} \mathrm{NaCl}, 25.0 \mathrm{mM} \mathrm{NaHCO}$, $1.2 \mathrm{mM} \mathrm{MgSO}_{4}, 1.2 \mathrm{mM} \mathrm{NaH}_{2} \mathrm{PO}_{4}, 1.4 \mathrm{mM} \mathrm{CaCl}_{2}, 3 \mathrm{mM} \mathrm{KCl}$ (or $5 \mathrm{mM}$ where indicated), and $11.1 \mathrm{mM}$ glucose. Perfusion solution was delivered at $37^{\circ} \mathrm{C}$ and $\mathrm{pH} 7.4$; perfusion pressure was maintained constant at $70 \mathrm{~mm} \mathrm{Hg}$. The left main coronary artery was occluded by a silk suture for 30 min. A unipolar ECG was recorded using a MacLab system (ADInstruments Ltd., Oxford, UK)(15, 16, 22, 23).

\subsubsection{Drug administration protocol, groups of hearts}

In the first two sets of experiments, Krebs' solution contained $3 \mathrm{mM} \mathrm{K}^{+}$. In the third and fourth sets of experiments, $\mathrm{K}^{+}$concentration was elevated to $5 \mathrm{mM}$. Each set of experiments contained four groups of hearts: one control and three drug-treated groups, each treated with one concentration of a representative Class I antiarrhythmic (quinidine, lidocaine or flecainide). One lower and one higher concentration of quinidine $(0.79$ and $7.90 \mu \mathrm{M})$, lidocaine $(3.88$ and $12.93 \mu \mathrm{M})$, and flecainide $(0.74$ and $1.48 \mu \mathrm{M})$, representing the peak unbound plasma and total 
blood concentrations, respectively, at "therapeutic" dosage, were evaluated in the first two sets of experiments and also in the last two sets of experiments. Each group contained 12 hearts in the first two sets of experiments (96 hearts in total; $3 \mathrm{mM} \mathrm{K}^{+}$in the Krebs), whereas every group in the third and fourth sets of experiments contained 6 hearts (48 hearts in total, $5 \mathrm{mM}$ $\mathrm{K}^{+}$in the Krebs). Experiments were done in a randomized and blinded manner.

\subsubsection{Reanalysing ischaemic arrhythmias according to definitions of LC I and LC II,} measurement of the number and incidence of tachyarrhythmias

The ECG recordings of the original investigation done by Farkas and Curtis (15) were reanalysed by two independent investigators using LabChart7 (ADInstruments Ltd., Oxford, UK). The two investigators were well trained and equally experienced in evaluating ECG. Ventricular arrhythmias were defined according to the LC I for the primary evaluation (Table 1). The number of VT and VF episodes, the incidence and the time to onset of VT and VF were determined from the ECG recorded during the 30-min-long ischaemia. Then the whole analysis was repeated according to the arrhythmia definitions of LC II (Table 1.). All variables were measured in a blinded manner.

\subsubsection{Statistical analysis of the results}

Continuous variables were expressed as means \pm SEM. Within group comparison of continuous data (e.g. the number of arrhythmia episodes, the onset times of VT and VF) were performed with the non-parametric Wilcoxon test for paired samples. Arrhythmia incidences were expressed as percent values, and were compared with Fisher's exact test with the Bonferroni correction, that is, the $P$ values of Fisher's exact test were multiplied by the numbers of comparisons to allow multiple comparisons (23). A $P$ value $<0.05$ was taken as indicative of a statistically significant difference between values. The intra-observer agreement on arrhythmia incidence data obtained according to LC I and LC II was assessed with Cohen's kappa statistic (23). Similarly, the inter-observer agreement on VT and VF incidence data among independent observers was calculated with Cohen's kappa statistic (23). 


\section{RESULTS}

\subsection{Results of the first study - Understanding the relationship between ventricular cycle} length irregularity and coronary flow in Langendorff perfused guinea pig hearts

\subsubsection{First set of experiments, irregular rhythm affects coronary flow}

First, the beat-to-beat variability of the ventricular cycle length was tested as an independent variable influencing coronary flow. RMSSD values were significantly greater in the 'High' RR variability group than in the 'Low' RR variability group (Figure 2a). This reflects the intensity of arrhythmias; the per cent frequency of arrhythmic beats was significantly greater in the 'High' RR variability group versus the 'Low' RR variability group (per cent frequency in the 'High' RR variability group: mean 14, median 2.7, range 0-53 versus per cent frequency in the 'Low' RR variability group: mean: 0; median 0 range: $0-0.8 ; \mathrm{P}<0.05$ ). RR interval variability was inevitably increased by spontaneously occurring arrhythmias during the observed 30-second-long period in the 'High' RR variability group: VPBs occurred in 25 hearts (an average of 21 beats, median 5 beats, range 1-58 beats), however VPBs with short coupling interval (R-on-T VPBs) occurred very rarely (in 6 hearts, an average of 3 beats, median 2.5 beats, range 1-5 beats); APB-s occurred in 9 hearts (an average of 12 beats, median 4 beats, range $2-47$ beats); increased variability of RR intervals was caused only by sinus arrhythmia in 10 hearts. Hearts in the 'Low' RR variability group remained in regular sinus rhythm. 
Importantly, the mean ventricular rate did not differ significantly between the two groups (Figure $2 b$ ), whereas the coronary flow was significantly greater in the 'High' RR variability group (Figure $2 c$ ).

a.

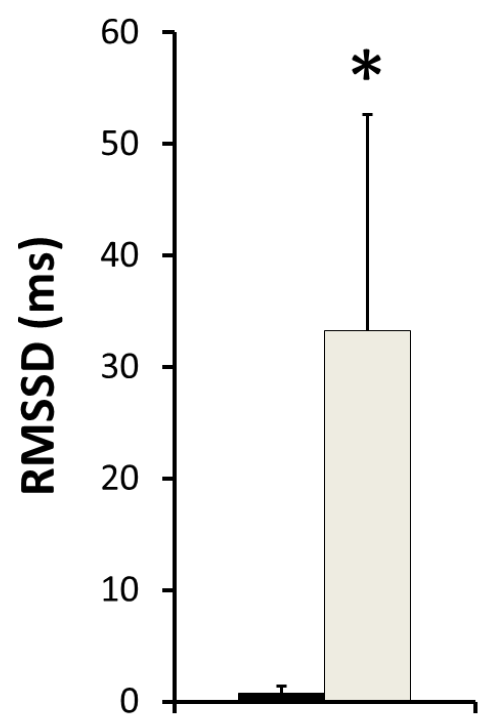

b.

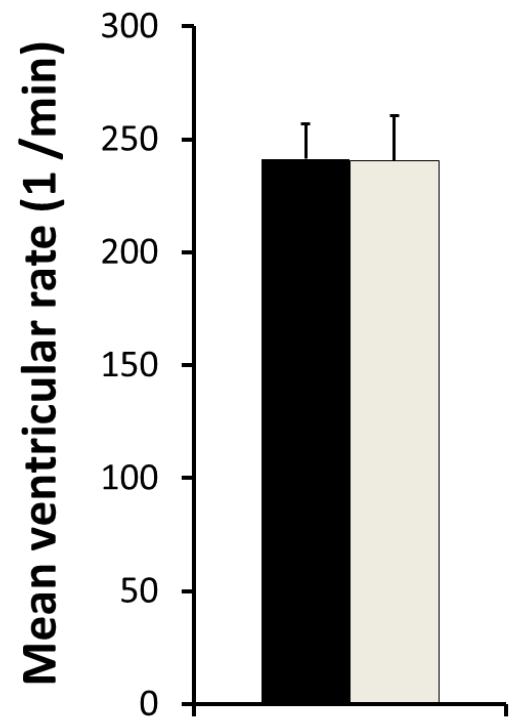

C.

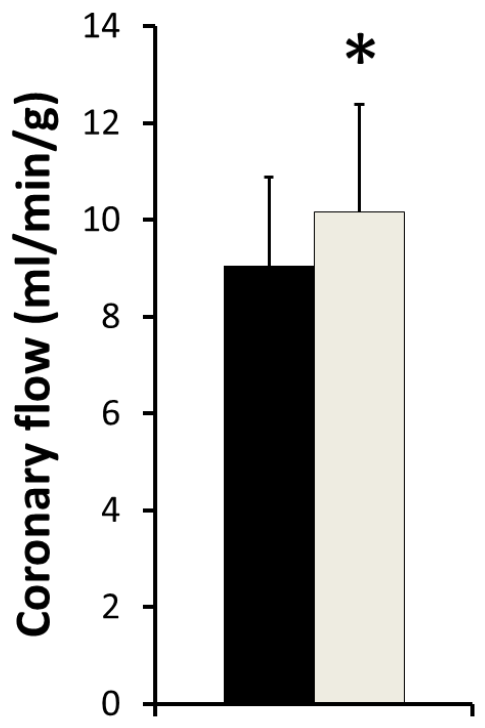

Figure 2. Beat-to-beat variability of the ventricular cycle length (RMSSD) (part a), mean ventricular rate (part b) and coronary flow (part c) in the 'Low' and 'High' RR variability groups in the first set of experiments. The dark columns illustrate the 'Low' RR variability group; light columns illustrate the 'High' $\mathrm{RR}$ variability group. ${ }^{*} \mathrm{P}<0.05$ vs. 'Low' RR variability group. For further details, see Figure 1. 
The relationship between mean ventricular rate and coronary flow was tested in a further analysis. A significant positive linear correlation was found between these variables in the 'Low' RR variability group, but with a low correlation coefficient (Figure 3.). A similar relationship was seen in the 'High' RR variability group (Figure 3.). However, the slope of the regression line was significantly greater in the 'High' versus 'Low' RR variability group, with consequentially greater coronary flow values in the 'High' RR variability group in the physiological heart rate range for guinea pig (210-280 1/min) (Figure 3.).

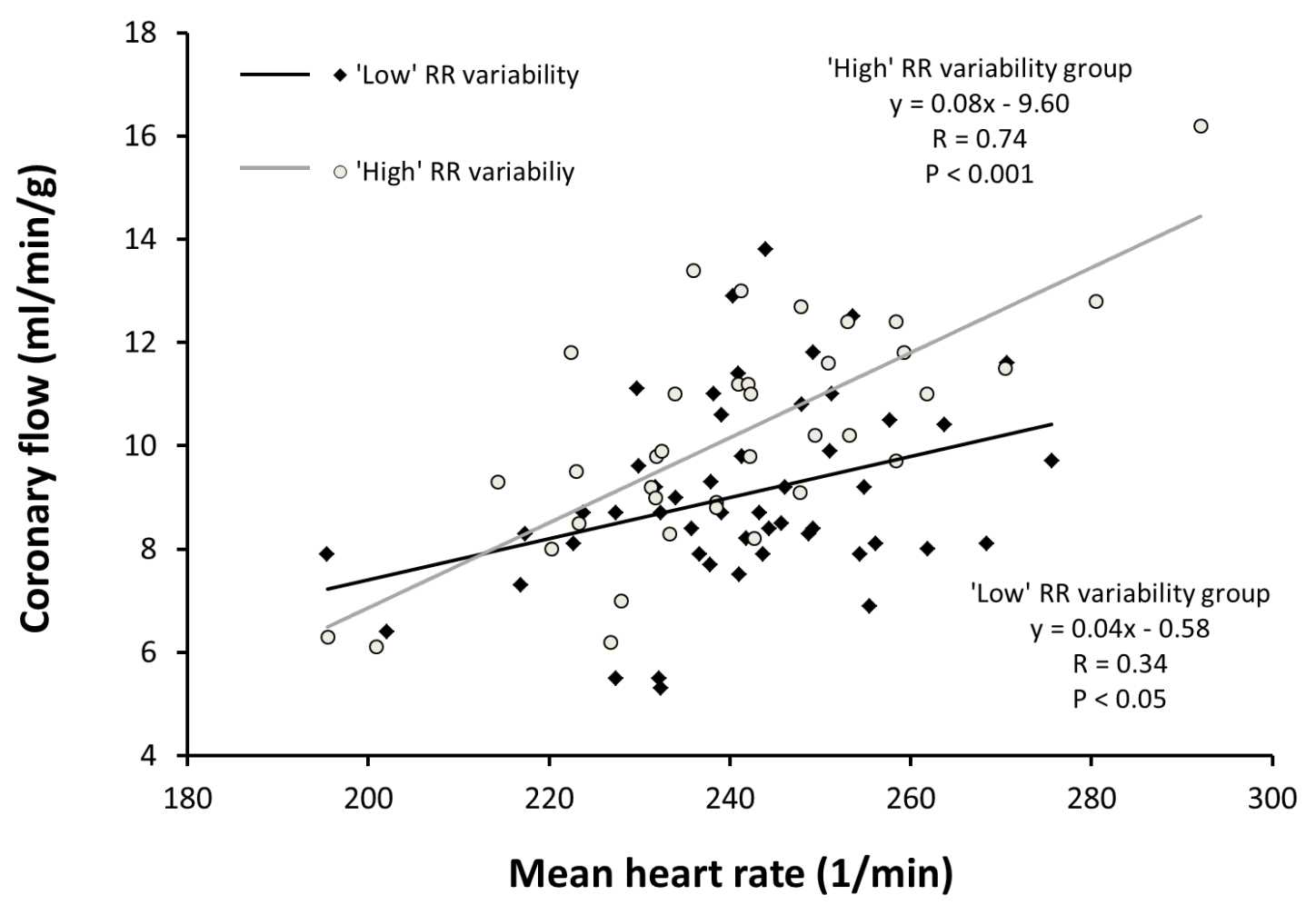

Figure 3. Correlation between mean ventricular rate and coronary flow in the 'Low' and 'High' RR variability groups in the first set of experiments. The slope of the regression line of the 'High' RR variability group is significantly greater than that of the 'Low' RR variability group ( 0.08 vs. 0.04 , respectively; ${ }^{*} \mathrm{P}<0.05$, analysis of covariance).

\subsubsection{Second set of experiments; duration of perfused and non-perfused intervals}

The RMSSD, mean ventricular rate and coronary flow values measured in the first set of experiments were reproduced in the second set of experiments. Accordingly, the beat-to-beat variability of the ventricular cycle length was significantly greater in the 'High' RR variability group than in the 'Low' RR variability group (RMSSD: $29.4 \pm 16.2 \mathrm{~ms}$ vs. $1.0 \pm 0.5 \mathrm{~ms}$, respectively; $\mathrm{P}<0.05)$. Mean ventricular rate did not differ between the 'High' and 'Low' RR 
variability groups ( $240 \pm 7$ vs. $236 \pm 41 / \mathrm{min}$, respectively). Mean aortic flow (which strongly correlated with coronary flow; see methods) was significantly greater in the 'High' RR variability group than in the 'Low' $\mathrm{RR}$ variability group $(8.9 \pm 1.8 \mathrm{ml} / \mathrm{min} / \mathrm{g}$ vs. $6.8 \pm 1.1$ $\mathrm{ml} / \mathrm{min} / \mathrm{g}$, respectively; $\mathrm{P}<0.05$ ). 
The analysis of the real time aortic flow signal, the perfusion pressure and the left ventricular pressure in each cardiac cycle revealed that left ventricular pressure exceeded perfusion pressure in most beats in hearts with 'Low' RR variability, associated with a transient reversal of the direction of aortic flow (Figure 4.). The average (per beat) duration of the nonperfused interval (in which left ventricular pressure exceeded perfusion pressure) did not differ significantly between the 'High' and 'Low' RR variability groups ( $37 \pm 10 \mathrm{~ms}$ vs. $38 \pm 8 \mathrm{~ms}$, respectively).

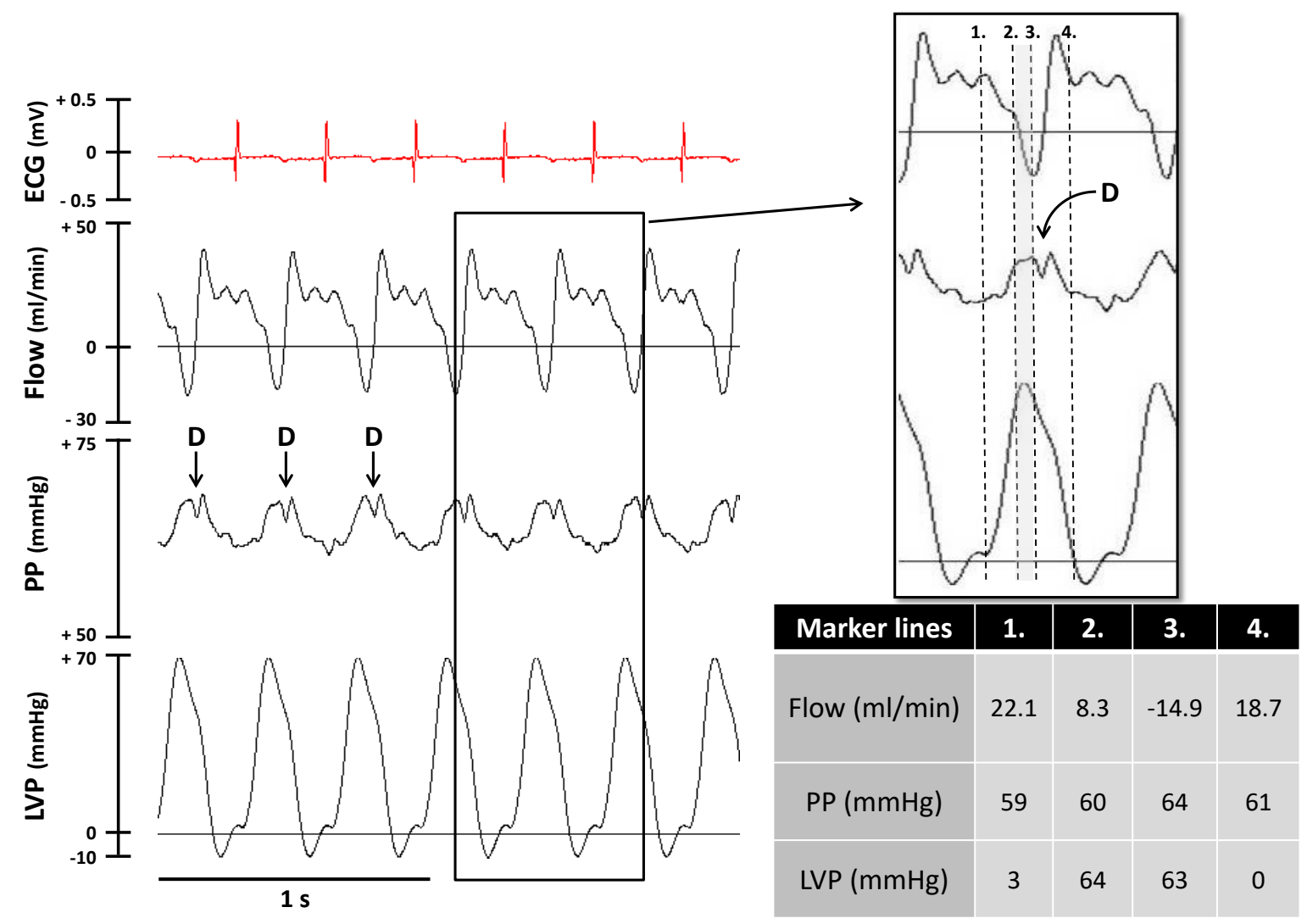

Figure 4. The volume conducted electrocardiogram (ECG), real-time aortic flow (Flow), the perfusion pressure (PP) and the left ventricular pressure (LVP) signals in regular sinus rhythm in an isolated, Langendroff perfused guinea pig heart. D: dicrotic notch in the perfusion pressure signal. A short segment of the flow, perfusion pressure and left ventricular pressure signals in the box is magnified in the right hand site. Table shows the corresponding values of the flow, perfusion pressure and left ventricular pressure at the dashed, vertical marker lines in the magnified segment. Note that left ventricular pressure exceeds perfusion pressure during systole in the grey area between marker lines 2-3 meaning that coronary artery perfusion is compromised by intramural pressure during this period. Also, real-time aortic flow signal shows negative values during this non-perfused period meaning that left ventricle ejects into the aortic stump against the perfusion column, when left ventricular pressure overcomes perfusion pressure. Closure of the aortic valve at the end of ejection is shown by the dicrotic notch in the perfusion pressure signal, when left ventricular pressure becomes lower than perfusion pressure immediately after marker line 3 . Note that atrial activity ( $\mathrm{P}$ wave) is not recognizable in the volume conducted ECG signal as only ventricles were submerged. 
However, cumulative number of beats that lacked non-perfused interval was greater in the 'High' RR variability group versus in the 'Low' RR variability group (Figure 5a).

a.

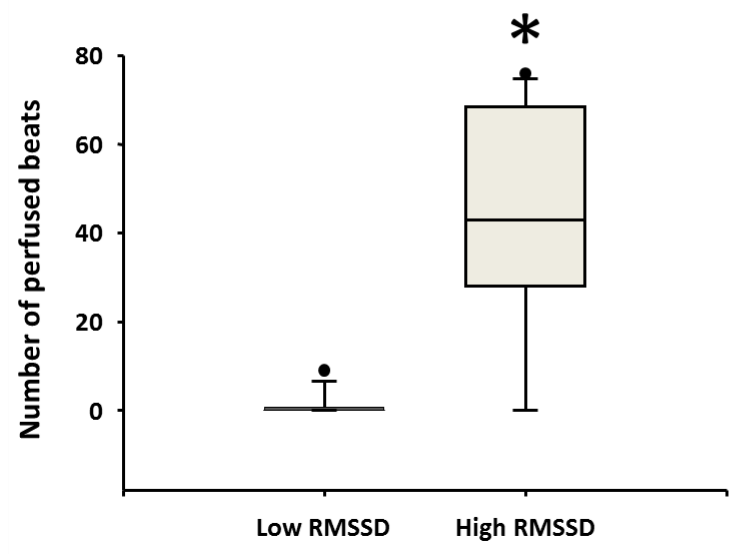

b.

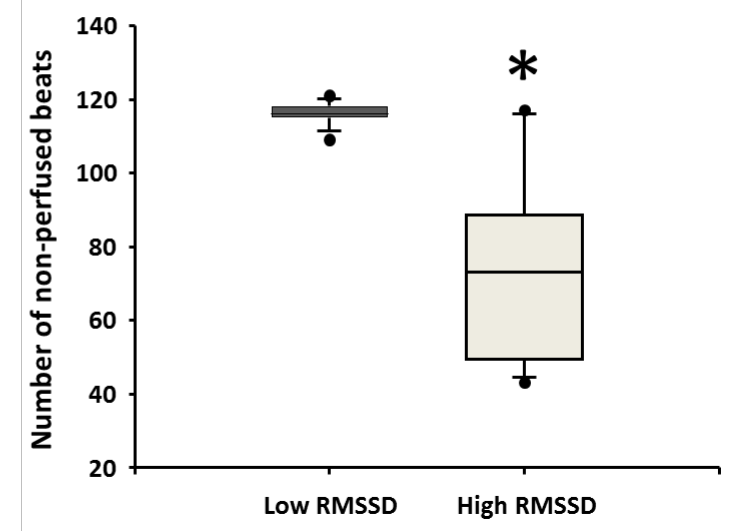

Figure 5. The number of perfused and non-perfused beats during the examined 30-second-long interval in the 'Low' and 'High' RR variability groups in the second set of experiments $(n=9$ hearts per group); box and whisker plots. Part a: the number of perfused beats, that is, the number of beats, in which left ventricular pressure remained below the perfusion pressure during systole. Part $b$ : the number of non-perfused beats, that is, the number of beats, in which left ventricular pressure exceeded perfusion pressure during systole. ${ }^{*} \mathrm{P}<0.05$ vs. 'Low' RR variability group. 
Consequently, the cumulative duration of the perfused intervals was significantly greater in the 'High' RR variability group versus in the 'Low' RR variability group (Figure 6a). Thus, ventricular irregularity significantly increased the ratio of the perfused to non-perfused intervals (Figure 6c). Usually, the beats that lacked non-perfused interval were atrial or ventricular premature beats and beats that follow 'post-extrasystolic potentiation' (PESP) beats (Figure 7.).

a.

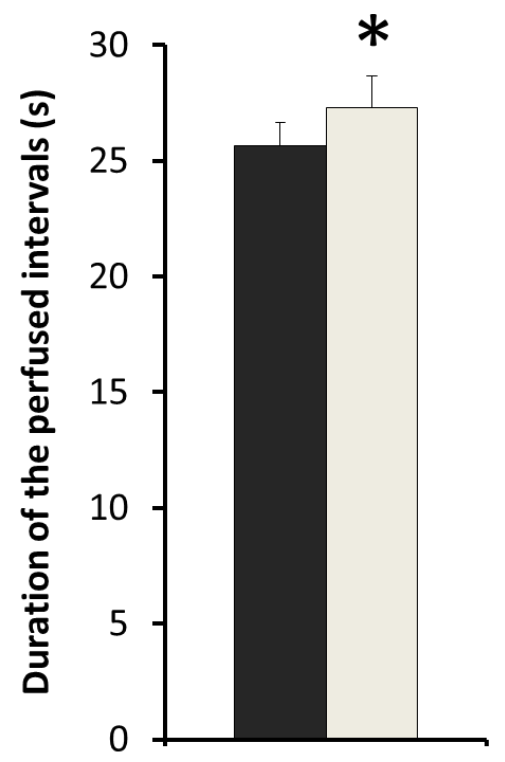

b.

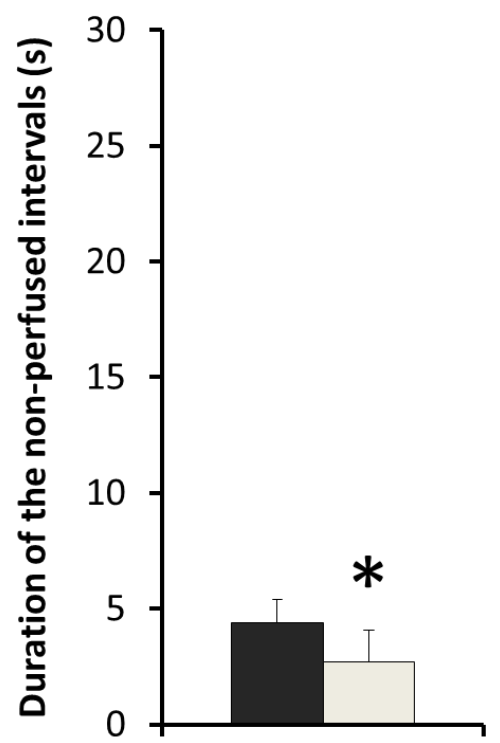

C.

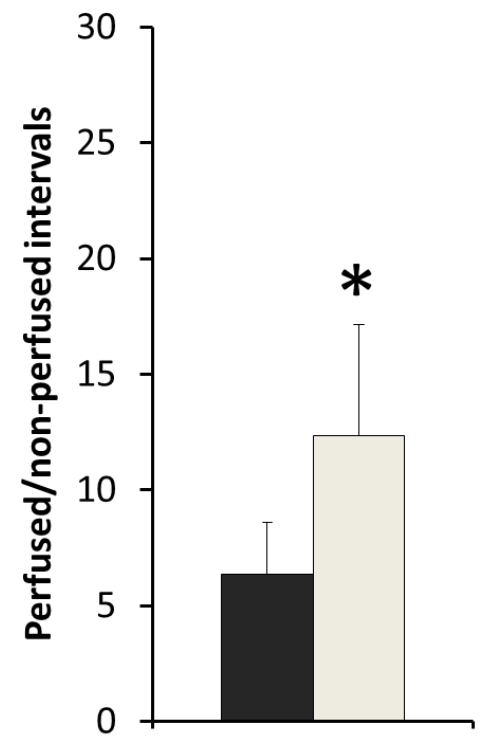

Figure 6. The cumulative duration of the perfused (part a) and non-perfused (part b) intervals in the examined 30-second-long period, and the ratio of the perfused to non-perfused intervals (part c) in the second set of experiments. The dark columns illustrate the 'Low' RR variability group; light columns illustrate the 'High' RR variability group. ${ }^{*} \mathrm{P}<0.05$ vs. 'Low' RR variability group. For further details, see Figure 5. 


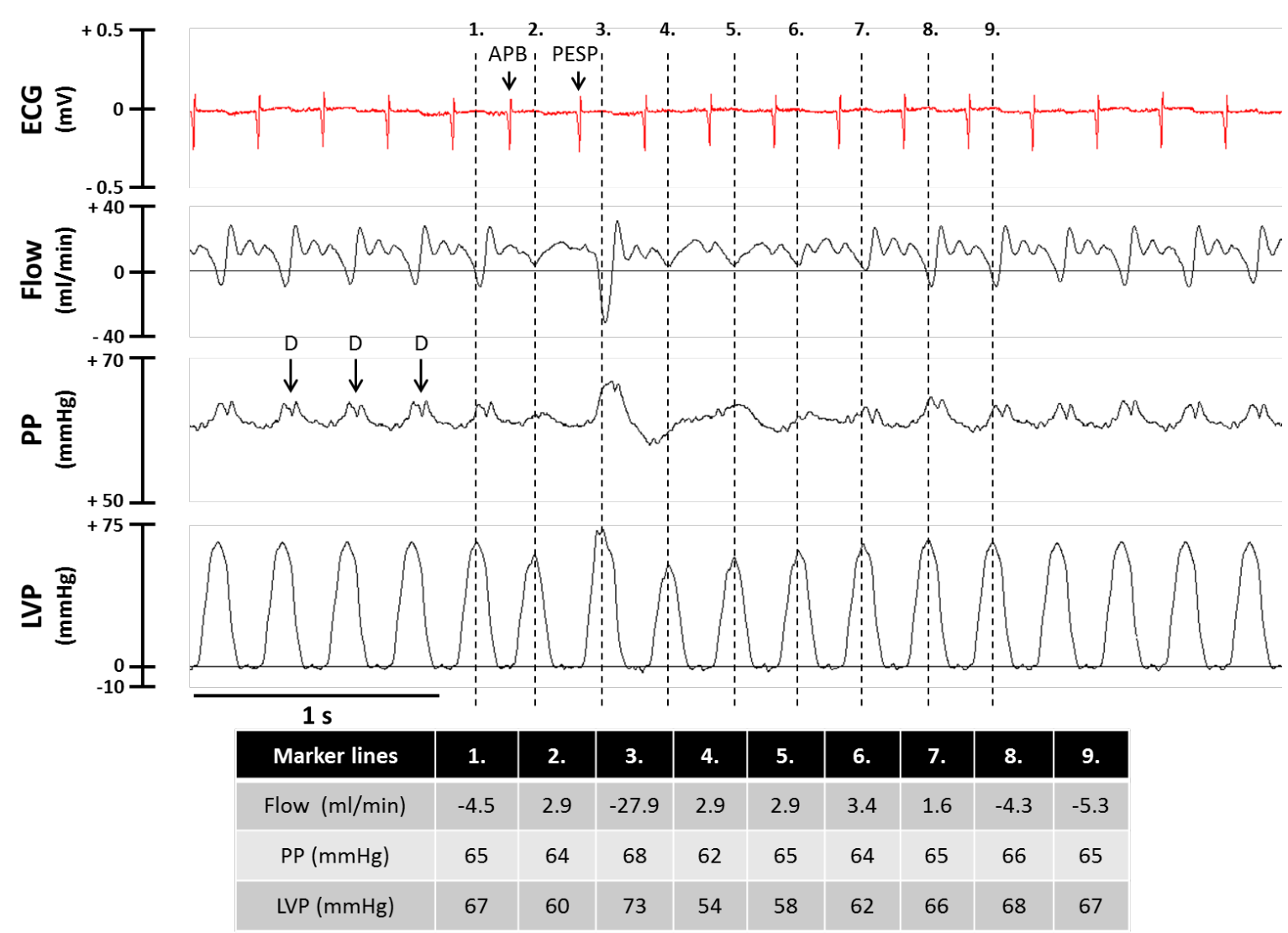

Figure 7. The effect of an atrial premature beat (APB) on the aortic flow (Flow), the perfusion pressure (PP) and the left ventricular pressure (LVP) in an isolated, Langendroff perfused guinea pig heart. ECG: volume conducted electrocardiogram, D: dicrotic notch in the perfusion pressure signal. Table shows the corresponding values of the flow, perfusion pressure and left ventricular pressure at the dashed, vertical marker lines. For further details see Figure 4. Note that the maximal left ventricular pressure value of the premature beat (at marker line 2) is lower than the corresponding value of the perfusion pressure. Premature beat is followed by a 'postextrasystolic potentiation' (PESP) beat, in which left ventricle produces a high peek ventricular pressure and a massive ejection (see values at marker line 3). PESP beat is followed by 3 beats in which the peek left ventricle pressure remains under the perfusion pressure, though left ventricular pressure increases gradually (see values at marker lines 4-6). Also note that coronary flow remains in the positive range in all 4 beats in which left ventricular pressure remains below the perfusion pressure (see values at marker lines 2, 4-6). This means that left ventricle does not eject in these 4 beats, and this is confirmed by the lack of the dicrotic notch (the sign of closure of the aortic valve) in the perfusion pressure signal in the beats at marker lines $2,4,5$ and 6; coronary artery perfusion is not compromised by the intramural pressure during the entire duration of these 4 beats. 
3.2. Result of the second study -Comparing arrhythmia results obtained according to the old and the updated Lambeth Conventions, validation of arrhythmia definitions

3.2.1. Ischaemic VF incidence in the first set of experiments $(3 \mathrm{mM} \mathrm{K}$, low concentrations of drugs)

When the arrhythmia definitions of LC I were applied, both of the two independent investigators found that VF was frequently induced in the control group, and only flecainide was able to reduce VF incidence significantly at the applied low concentration (Figure 8.). These results are in a good accordance with the arrhythmia results of the original investigation obtained by a different observer using the same arrhythmia definitions of LC I (12). This means that applying arrhythmia definitions of LC I allowed the two independent investigators to reproduce previous results.

When arrhythmia definitions of LC II were applied, both of the two independent investigators found that VF was induced in all control hearts, and none of the drugs affected significantly VF incidence at the applied low concentrations (Figure 8.). Thus, the significant antifibrillatory effect of the low concentration of flecainide was masked when arrhythmia definitions of LC II were used. This means that results about the antifibrillatory effect of flecainide qualitatively differ from those obtained by applying arrhythmia definitions of LC I. 

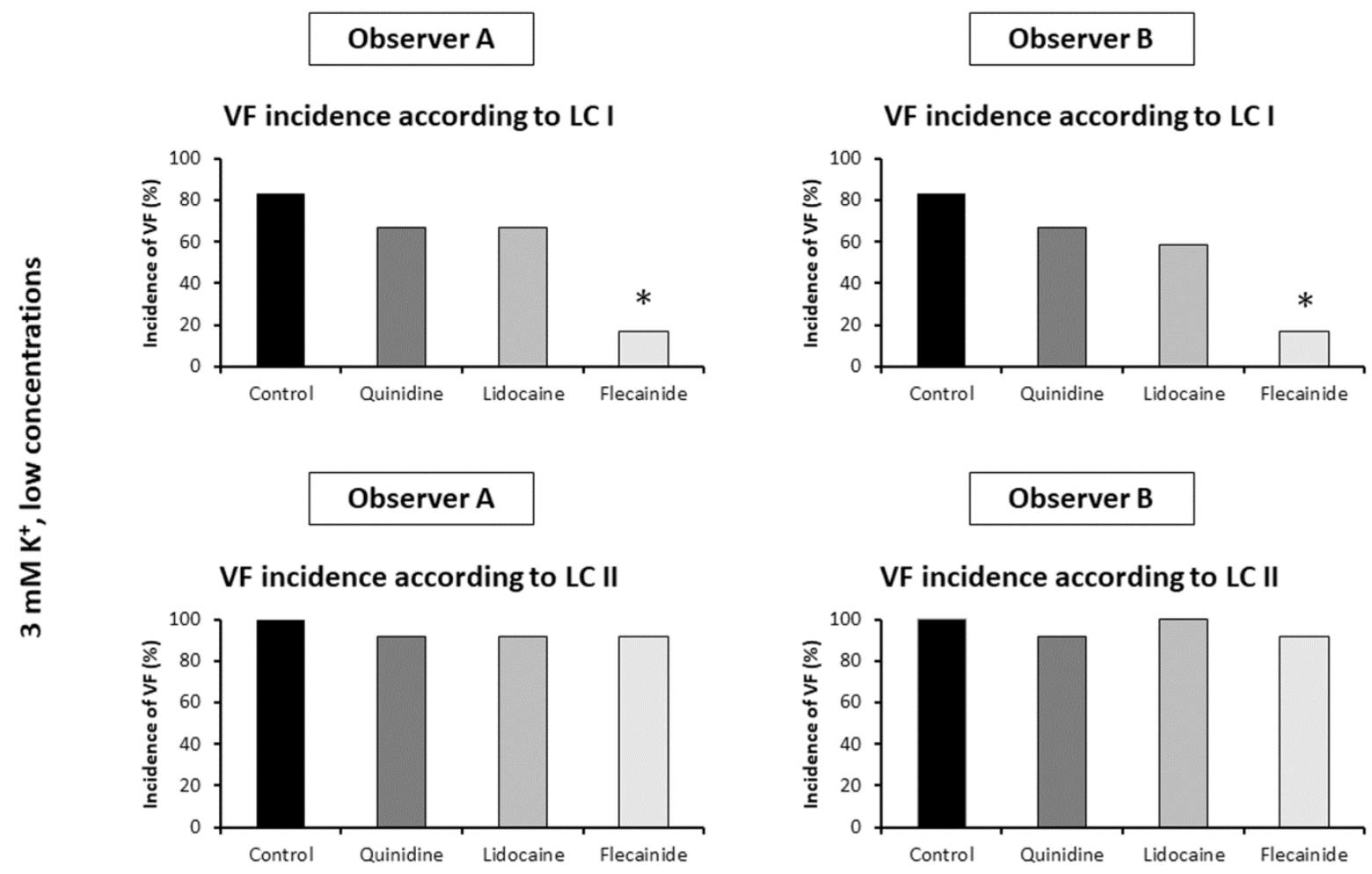

Figure 8. Comparison of the percent incidences of ischaemic ventricular fibrillation (VF) obtained from the 1st set of experiments by two independent observers (Observer A and Observer B) using VF definition of Lambeth Conventions I (LC I) and Lambeth Conventions II (LC II). Langendorff perfused rat hearts were subjected to local ischaemia for $30 \mathrm{~min}$. Hearts were perfused with the low concentration of quindine $(0.79 \mu \mathrm{M})$, lidocaine $(3.88 \mu \mathrm{M})$, flecainide (0.74) or solvent (Control), each group contained $n=12$ hearts, Krebs solution contained $3 \mathrm{mM} \mathrm{K}^{+}$. ${ }^{*} \mathrm{p}<0.05$ vs. Control.

\subsubsection{Ischaemic VF incidence in the second set of experiments $\left(3 \mathrm{mM} \mathrm{K} \mathrm{K}^{+}\right.$, high concentrations} of drugs)

When the arrhythmia definitions of LC I were applied, both of the two independent investigators found that VF was frequently induced in the control group, and all three drugs reduced VF incidence significantly at the applied high concentrations (Figure 9.). These results well accord with the arrhythmia results of the original investigation obtained by a different observer using the same arrhythmia definitions of LC I (12). This means that applying arrhythmia definitions of LC I allowed the two independent investigators to reproduce previous results.

When the arrhythmia definitions of LC II were applied, Observer B found that the high concentrations of all three drugs reduced the incidence of ischaemic VF as compared with control, which accords with the results obtained according to LC I (Figure 9.). However, Observer A found that the applied high concentration of flecainide did not significantly reduce 
the incidence of ischaemic VF, which qualitatively differs from the results obtained according to LC I, and it also contradicts the results of Observer B (Figure 9.).
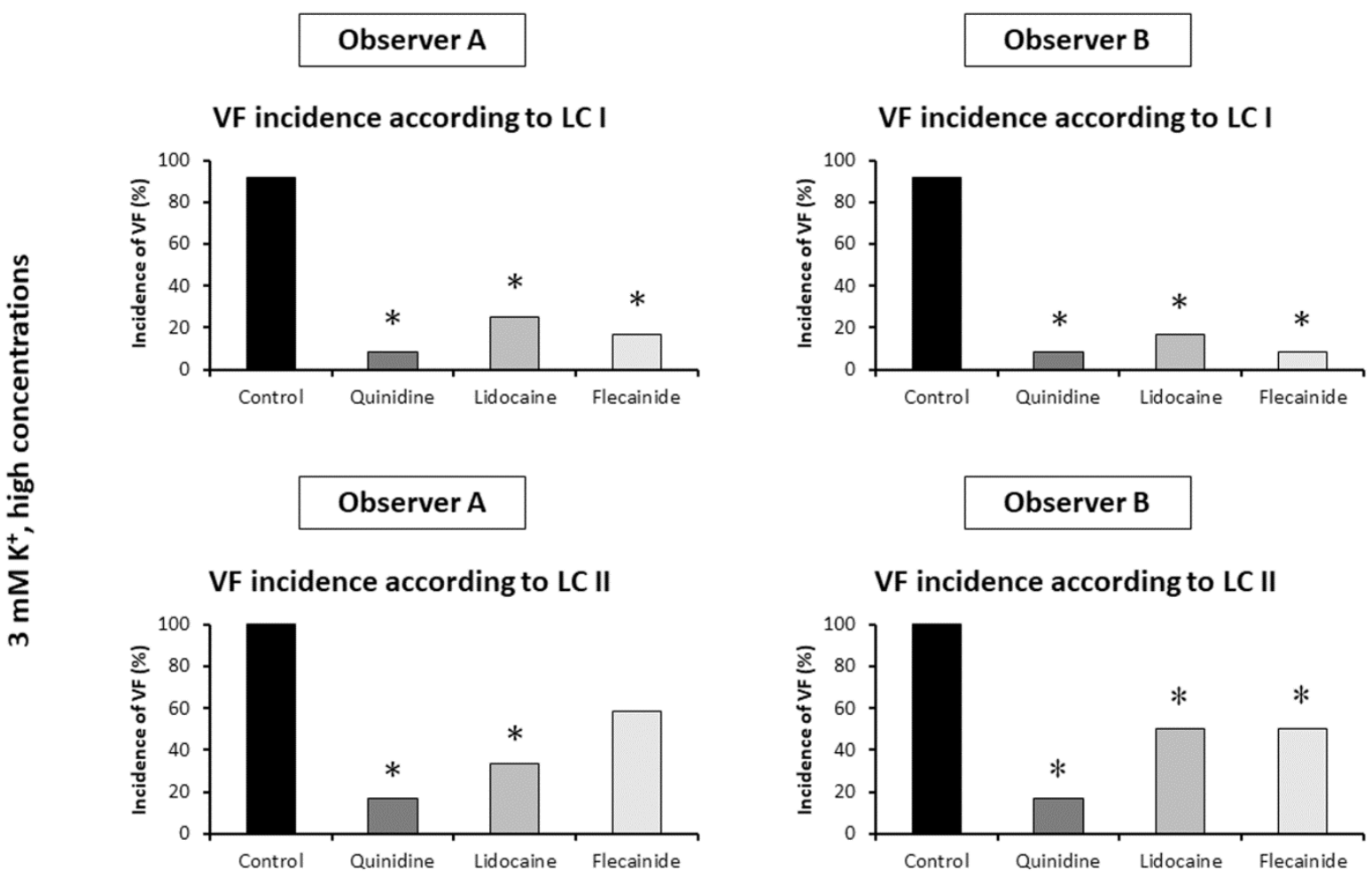

Figure 9. Comparison of the percent incidences of ischaemic ventricular fibrillation (VF) obtained from the 2nd set of experiments by two independent observers (Observer A and Observer B) using VF definition of Lambeth Conventions I (LC I) and Lambeth Conventions II (LC II). Langendorff perfused rat hearts were subjected to local ischaemia for $30 \mathrm{~min}$. Hearts were perfused with the high concentration of quindine $(7.90 \mu \mathrm{M})$, lidocaine $(12.93$ $\mu \mathrm{M})$, flecainide $(1.48 \mu \mathrm{M})$ or solvent (Control), each group contained $\mathrm{n}=12$ hearts, Krebs solution contained $3 \mathrm{mM} \mathrm{K}^{+} .{ }^{*} \mathrm{p}<0.05$ vs. Control.

\subsubsection{Ischaemic VF incidence in the control hearts, the effect of $K^{+}$concentration on VF} incidence

Ischaemic VF incidence was compared between the control group of hearts perfused with $3 \mathrm{mM} \mathrm{K}^{+}$(the hearts of the control groups of the 1st and 2nd sets of experiments) and the control hearts perfused with $5 \mathrm{mM} \mathrm{K}^{+}$(the hearts of the control groups of the 3rd and 4th sets of experiments). Both investigators found that the elevation of the $\mathrm{K}^{+}$concentration from 3.0 $\mathrm{mM}$ to $5.0 \mathrm{mM}$ significantly reduced the incidence of ischaemic VF, when arrhythmia definitions of LC I were applied (Figure 10.). These results well accord with the arrhythmia results of the original investigation obtained by a different observer using the same arrhythmia definitions of LC I (12). Again, this shows that applying arrhythmia definitions of LC I allowed the two independent investigators to reproduce previous results. However, the marked antiarrhythmic effect of the increased $\mathrm{K}^{+}$concentration seen according to LC I was masked 
when arrhythmia definitions of LC II were applied in the present investigation, and these results did not differ qualitatively between the two investigators (Figure 10.).

\section{Observer A}

VF incidence according to $\mathrm{LCI}$

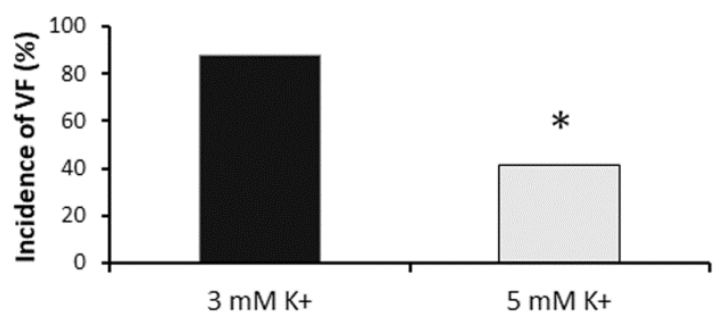

Observer A

VF incidence according to LC II

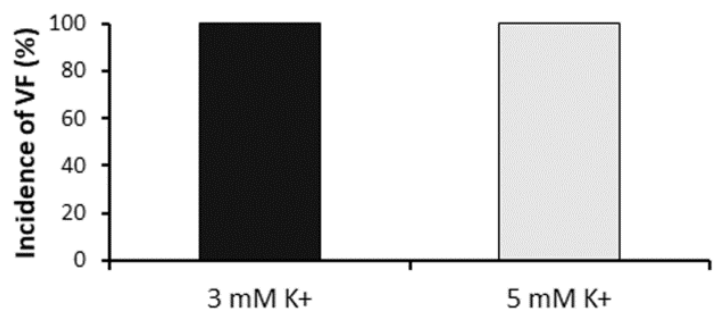

Observer B

VF incidence according to $\mathrm{LCI}$

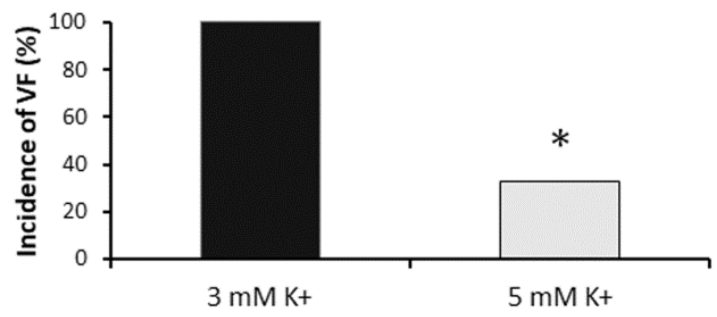

Observer B

VF incidence according to LC II

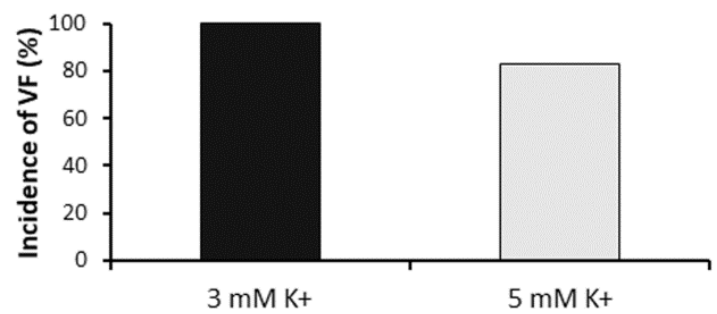

Figure 10. Comparison of the percent incidences of ischaemic ventricular fibrillation (VF) between control rat hearts subjected to local ischaemia for 30 min and perfused with Krebs solution containing 3 or $5 \mathrm{mM} \mathrm{K}^{+}$( $\mathrm{n}=24$ and $\mathrm{n}=12$ hearts, respectively). Two independent observers (Observer A and Observer B) performed the blinded arrhythmia analysis using VF definition of Lambeth Conventions I (LC I) and Lambeth Conventions II (LC II). ${ }^{*} p<0.05$ vs. $3 \mathrm{mM} \mathrm{K}^{+}$.

\subsubsection{Ischaemic VT and VF incidences in the whole investigation, and intra-observer}

\section{agreement}

VT and VF incidences were calculated irrespective of the treatment and the $\mathrm{K}^{+}$content of the perfusate in the 144 hearts of the whole investigation. Observer A did not find significant effect of LC II on VT incidence, and achieved good intra-observer agreement on VT incidence (Table 2.). However, Observer $\mathrm{B}$ found that applying arrhythmia definitions of LC II significantly reduced VT incidence as compared with the respective value obtained according to LC I, which resulted in only moderate intra-observer agreement on VT incidence (Table 2.). 
Table 2. The percent incidences of ischaemic ventricular tachycardia and ventricular fibrillation irrespective of the drug treatment and $\mathrm{K}^{+}$content of the perfusate in $\mathrm{n}=144$ Langendorff perfused rat hearts subjected to local ischaemia for $30 \mathrm{~min}$. Intra-observer agreement on the results obtained according to the arrhythmia definitions of Lambeth Conventions I and Lambeth Conventions II, results of the Cohen's Kappa statistical analysis.

\begin{tabular}{|cc|cc|c|c|}
\hline Arrhythmia & Observer & \multicolumn{2}{|c|}{ Incidence (\%) } & Kappa & 95\% Confidence Interval \\
& & LC I & LC II & & for kappa \\
\hline VT & A & 85 & 84 & 0.974 & $0.922-1.000$ \\
& B & 88 & $76^{*}$ & 0.586 & $0.421-0.751$ \\
\hline VF & A & 35 & $60^{*}$ & 0.529 & $0.411-0.646$ \\
& B & 33 & $60 *$ & 0.482 & $0.365-0.599$ \\
\hline
\end{tabular}

VT: ventricular tachycardia. VF: ventricular fibrillation. Observers A and B are the two independent observers of the present investigation. LC I and LC II, VT and VF incidence data obtained according to the arrhythmia definitions of Lambeth Conventions I and Lambeth Conventions II, respectively. Kappa: kappa coefficient obtained by Cohen's Kappa statistical analysis; note that kappa value of 1.0 means perfect agreement, whereas the kappa value of 0.0 means absolutely no agreement; thus, the greater the kappa value in the range of $0.0-1.0$, the greater the agreement. ${ }^{*} \mathrm{p}<0.05$ vs. LC I.

Both observers found that VF was diagnosed in a significantly greater proportion of hearts, when the arrhythmia definitions of LC II were applied, as compared with the respective value measured according to the arrhythmia definitions of LC I (Table 2.). Also, both observers found that when arrhythmia definitions of LC II were applied, VF was diagnosed in all hearts, in which VF was diagnosed according to LC I. However, there were many hearts, in which VF was diagnosed only according to LC II and VF was not found according to LC I (Figure 11.). Consequently, Cohen's Kappa analysis showed that intra-observer agreement on VF incidence data obtained according to LC I and LC II was only moderate in case of both A and B Observers (Table 2.). 


\section{Observer A}

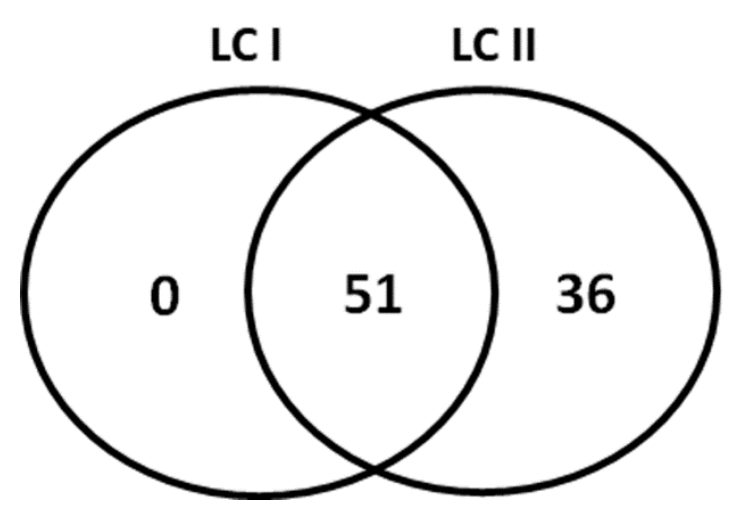

\section{Observer B}

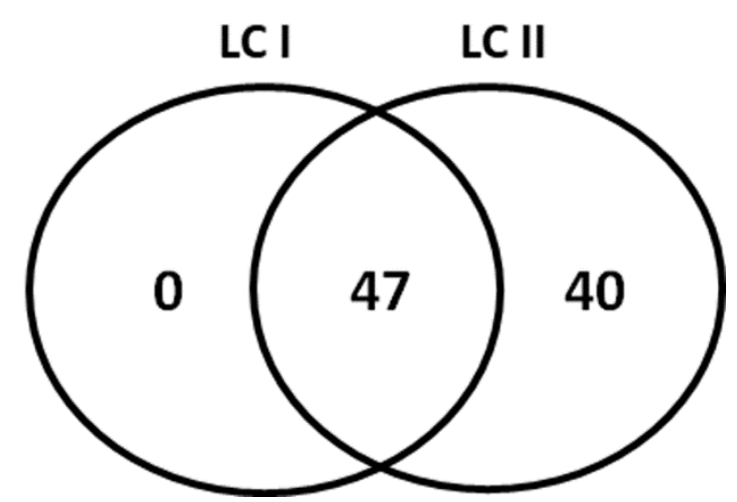

Figure 11. The total number of hearts with ischaemic ventricular fibrillation (VF) out of the 144 Langendorff perfused rat hearts subjected to local ischaemia for $30 \mathrm{~min}$ irrespective of the drug treatment and the $\mathrm{K}^{+}$content of the perfusate. VF was diagnosed by applying VF definitions of Lambeth Conventions I (LC I) and Lambeth Conventions II (LC II). Observer A and Observer B are the two independent observers. The number in the common part of the LC I and LC II classes shows the total number of hearts that experienced VF according to both LC I and LC II.

\subsubsection{The inter-observer agreement on VT and VF incidences among independent observers}

The inter-observer agreement on VT and VF incidences among independent observers was calculated irrespective of the treatment and the $\mathrm{K}^{+}$concentration in the 144 hearts of the whole investigation. Applying VT definition of LC II did not remarkably improve the interobserver agreement on VT incidence between Observers A and B; moderate inter-observer agreement was found between them when VT incidence was tested either according to LC I or according to LC II (Table 3.).

Importantly, when VF incidence obtained according to LC I was tested, very strong inter-observer agreements (high kappa values) were found among the two independent investigators (Observers A and B) and the investigator of the original investigation ['Observer O',(12)] (Table 3.). When arrhythmia definitions of LC II were applied, a good agreement was found in VF incidence between the two independent investigators; however, the kappa value was lower than that obtained according to LC I (Table 3.). 
Table 3. Inter-observer agreement on the incidences of ventricular tachycardia and ventricular fibrillation. Results of the Cohen's Kappa statistical analysis.

\begin{tabular}{|c|c|c|c|c|}
\hline Incidence of & Lambeth Convention & Observers & Kappa & $\begin{array}{c}95 \% \text { Confidence Interval } \\
\text { for kappa }\end{array}$ \\
\hline \multirow{3}{*}{ VT } & \multirow{2}{*}{ LC I } & A vs. O & 0.918 & $0.826-1.000$ \\
\cline { 3 - 5 } & & B vs. O & 0.615 & $0.424-0.805$ \\
\cline { 2 - 5 } & A vs. B & 0.652 & $0.471-0.833$ \\
\cline { 2 - 5 } & LC II & A vs. B & 0.675 & $0.525-0.825$ \\
\hline \multirow{4}{*}{ VF } & \multirow{4}{|l}{} \\
\cline { 3 - 5 } & LC I & A vs. O & 0.908 & $0.836-0.980$ \\
\cline { 2 - 5 } & B vs. O & 0.938 & $0.877-0.998$ \\
\cline { 2 - 5 } & LC II & A vs. B & 0.938 & $0.879-0.998$ \\
\cline { 2 - 5 } & A vs. B & 0.884 & $0.806-0.962$ \\
\hline
\end{tabular}

VT: ventricular tachycardia. VF: ventricular fibrillation. LC I and LC II: VF and VT incidence data obtained according to the arrhythmia definitions of Lambeth Conventions I and Lambeth Conventions II, respectively. Observers A and B are the two independent observers of the present investigation, Observer $\mathrm{O}$ is the observer of the original investigation published by Farkas and Curtis (15). Kappa: kappa coefficient obtained by Cohen's Kappa statistical analysis. VT and VF incidences were obtained irrespective of the drug treatment and $\mathrm{K}^{+}$ content of the perfusate from $\mathrm{n}=144$ Langendorff perfused rat hearts subjected to local ischaemia for $30 \mathrm{~min}$.

\subsubsection{The onset time of ischaemic VF in the 1st set of experiments}

When hearts were perfused with the low concentrations of the drugs and Krebs solution contained $3 \mathrm{mM} \mathrm{K}$ (first set of experiments), VF incidences were high in the Control, Quinidine and Lidocaine groups of hearts irrespective of the applied VF definition (see Figure 8.). This allowed us to examine the effect of the applied arrhythmia definitions on the onset time of the first episode of ischaemic VF in these three groups. Both observers found that VF onset time was significantly reduced in these three groups, when arrhythmia definitions of LC II were used as compared with the respective values obtained by applying arrhythmia definitions of LC I (Figure 12a and Figure 12b). This shows that some ischaemic arrhythmias, which were not identified as VF according to LC I, were identified as VF according to LC II, and these arrhythmias were found not only in drug-free hearts but in quinidine and lidocaine treated hearts, too. When VT definition of LC II was applied, VT onset times were not significantly affected in any of the groups as compared with the respective values obtained by applying VT definition of LC I (data not shown). 
a

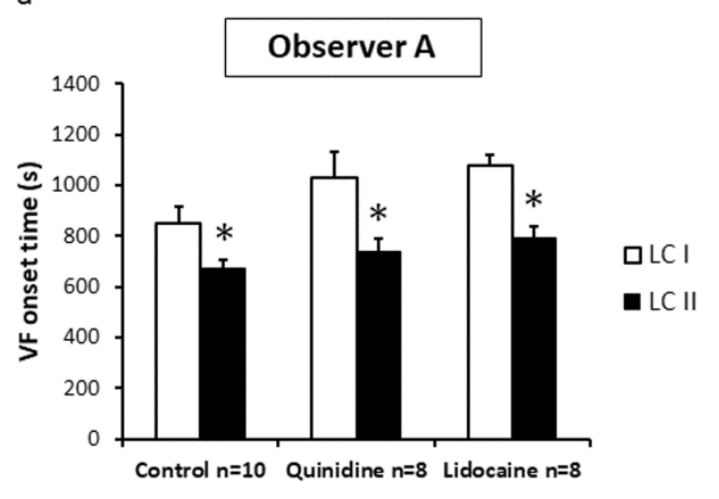

b

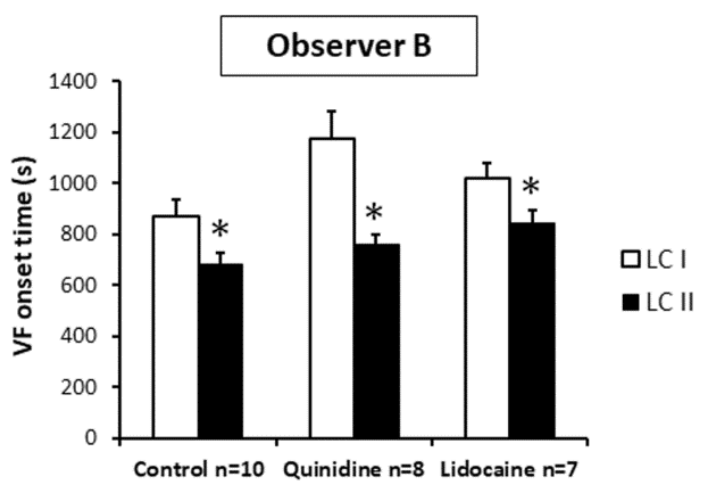

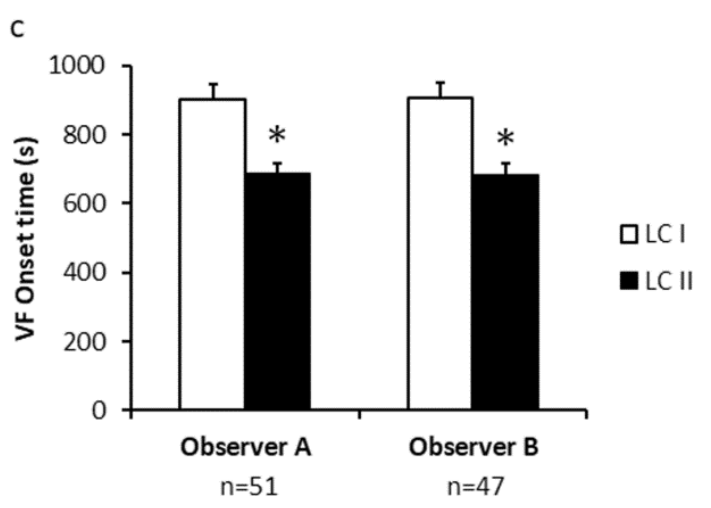

Figure 12. The effect of definitions of ventricular fibrillation (VF) of Lambeth Conventions I (LC I) and Lambeth Conventions II (LC II) on the onset time of the first episode of ischaemic VF in Langendorff perfused rat hearts subjected to local ischaemia for $30 \mathrm{~min}$ and experiencing VF according to both LC I and LC II. Parts a and b: VF onset times in the 1st set of experiments, in which hearts were perfused with the low concentration of quindine $(0.79$ $\mu \mathrm{M})$, lidocaine $(3.88 \mu \mathrm{M})$, or solvent (Control), Krebs solution contained $3 \mathrm{mM} \mathrm{K}^{+}$. Part c: VF onset times in the whole set of experiments irrespective of drug treatment and the $\mathrm{K}^{+}$ content of the perfusate. Observer A and Observer B are the two independent observers. $* \mathrm{p}<0.05$ vs. LC I.

\subsubsection{The onset time of $V F$ in all hearts that experienced $V F$ irrespective of $K^{+}$content of the}

\section{perfusate and drug treatment}

When all those hearts were examined that experienced ischemic VF according to both LC I and LC II in the whole investigation, the two independent observers found that applying arrhythmia definitions of LC II significantly reduced the onset time of the first episode of VF as compared with the value obtained by using arrhythmia definitions of LC I (Fig. 12c). Applying VF definition of LC II did not increase VF onset time in any of the hearts but reduced this variable in approximately $80 \%$ of the hearts (Table 4.). These results did not differ qualitatively between the two independent observers (Table 4.). 
Table 4. The effect of applying the new definition of ventricular fibrillation of Lambeth Conventions II on the onset time of ischaemic ventricular fibrillation in Langendorff perfused rat hearts.

\begin{tabular}{|l|c|c|c|c|}
\hline & \multicolumn{2}{|c|}{ Observer A } & \multicolumn{2}{c|}{ Observer B } \\
\hline & Number of hearts & $\%$ & Number of hearts & $\%$ \\
\hline & & & & \\
\hline VF found in the same heart by both LC I and LC II & 51 & 100 & 47 & 100 \\
\hline VF onset time reduced by LC II & 42 & 82 & 38 & 81 \\
\hline VF onset time not affected by LC II & 9 & 18 & 9 & 19 \\
\hline VF onset time increased by LC II & 0 & 0 & 0 & 0 \\
\hline
\end{tabular}

The onset time of ischaemic VF was examined in all hearts that experienced VF irrespective of $\mathrm{K}^{+}$content of the perfusate and drug treatment in the whole investigation (in all four sets of experiments utilising $\mathrm{n}=144$ isolated rat hearts). LC I and LC II, Lambeth Conventions I and II, respectively. VF, ischaemic ventricular fibrillation. Observer A and Observer B, the two independent observers who performed blinded arrhythmia analysis of the experiments.

\subsubsection{The numbers of VT and VF episodes}

In order to examine whether applying arrhythmia definitions of LC II causes an 'arrhythmia shift' from the VT class to the VF class, the numbers of VT and VF episodes were calculated in the control and the flecainide treated groups of the first set of experiments $(n=12$ hearts in each group). These two groups were chosen, as the greatest and least number of arrhythmias occurred in the Control and Flecainide groups, respectively. First, a statistical analysis was performed on the data of those eight hearts of the flecainide treated group of the first set of experiments, in which neither Observer A nor Observer B found VF according to LC I, but both of them identified VF according to LC II. In the flecainide treated 8 hearts, both observers found that applying VT definition of LC II reduced the number VT episodes as compared with the respective values obtained by using VT definition of LC I (Figure 13.). On the other hand, applying VF definition of LC II significantly increased the number of VF episodes as compared with the respective zero value obtained by using VF definition of LC I (Figure 13.). Interestingly; Observer A found that arrhythmia analysis according to LC II significantly increased the cumulative number of VT and VF episodes as compared with the respective value obtained according to LC I in the flecainide treated 8 hearts. This suggests that when arrhythmia analysis was performed according to LC II, VT episodes were not only shifted to the VF class but were also fragmented (i.e. broken up into shorter episodes due to containing diastolic pauses). Observer B did not find any significant effect of LC II on the cumulative number of VT and VF episodes (Figure 13.). Analysis of the number of the VT and VF episodes in the 12 hearts of the control group of the first set of experiments yielded qualitatively the same results despite identifying VF episodes not only by LC II but LC I, too (Figure 14.). 


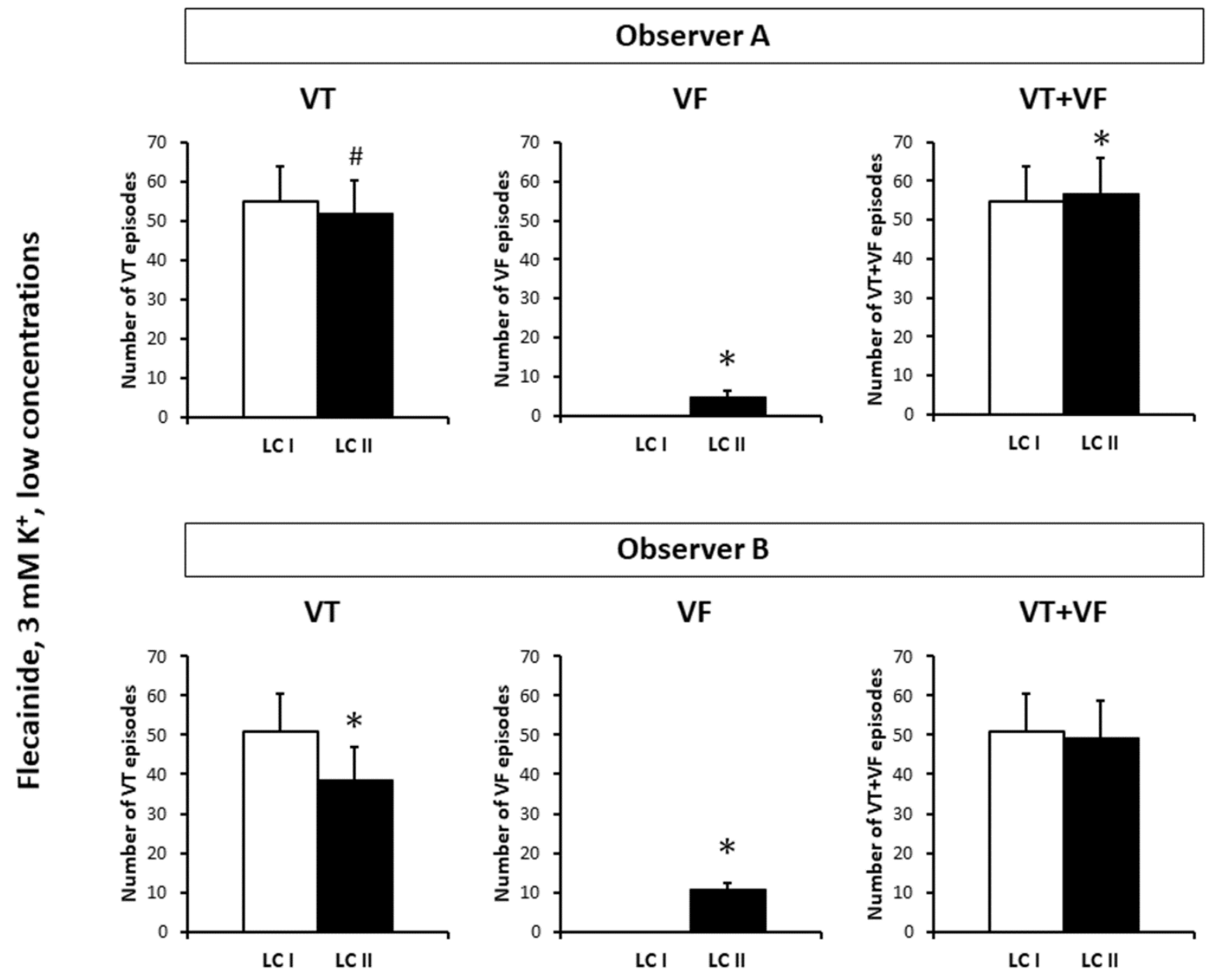

Figure 13. The numbers of ventricular tachycardia (VT) and ventricular fibrillation (VF) episodes and the cumulative number of VT and VF episodes (VT+VF) in those eight hearts of the flecainide treated group of the first set of experiments, in which neither Observer A nor Observer B found VF according to Lambeth Conventions I (LC I), but both of them identified VF according to Lambeth Conventions II (LC II). Observer A and Observer B are the two independent observers. ${ }^{*} p<0.05$ vs. LC I. $\# p=0.055$ vs. LC I. Note that VF episodes found by applying VF definition of LC II are 'de novo' VF episodes, and occurred only as a result of 'arrhythmia shift', and 'arrhythmia fragmentation' did not contribute to their occurrence. 


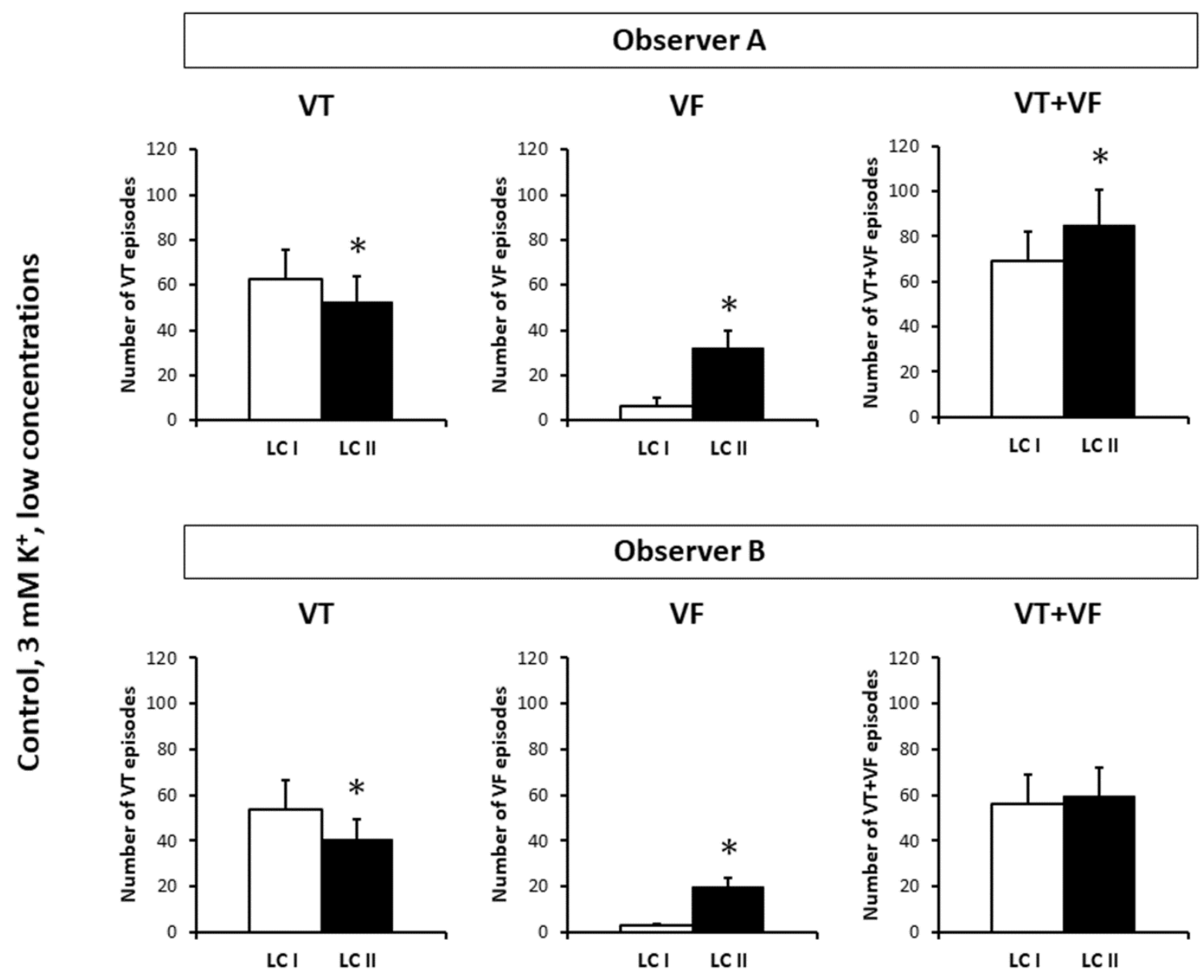

Figure 14. The numbers of ventricular tachycardia (VT) and ventricular fibrillation (VF) episodes and the cumulative number of VT and VF episodes (VT $+\mathrm{VF}$ ) in the $n=12$ control hearts of the first set of the experiments. LC I and LC II: data obtained by using arrhythmia definitions of Lambeth Conventions I and Lambeth Conventions II, respectively. Observer A and Observer B are the two independent observers. ${ }^{*} \mathrm{p}<0.05$ vs. LC I. Note, that the extra VF episodes found by applying VF definition of LC II can be the result of 'arrhythmia shift'. However, in theory, 'arrhythmia fragmentation' could also contribute to the increase in VF number according to LC II, as VF episodes found by LC I could be fragmented into shorter episodes due to containing diastolic pauses. 


\section{DISCUSSION}

\subsection{Understanding the correlation between increased beat-to-beat variability of ventricular cycle length and coronary flow in Langendorff perfused hearts}

There is a positive linear correlation between mean ventricular rate and coronary flow in Langendorff perfused guinea pig hearts. However, the slope of the regression line is steeper when beat-to-beat variability of ventricular cycle length increases, which results in an increase in coronary flow in the physiological heart rate range independently of mean RR interval.

In the present investigation with Langendorff perfused guinea pig hearts, left ventricular pressure at peak systole exceeded perfusion pressure. The positive effect of increased beat-tobeat variability of the cycle length on coronary flow resulted from the preponderance of beats in which left ventricular pressure did not exceed perfusion pressure during systole, facilitating coronary perfusion.

\subsubsection{Positive, linear correlation between the ventricular rate and coronary flow}

The linear correlation between mean ventricular rate and coronary flow reflects the established relationship between coronary blood flow in vivo and myocardial oxygen and nutrient demand $(5,6)$. One interesting implication from the present study is confirmation that autoregulation is largely autonomic-independent (Langendorff hearts are denervated). In vivo, the autonomic nervous system may influence autoregulation (6), but the relationship is preserved in autonomically denervated $\operatorname{dogs}(6,24)$, and in isolated, denervated, Langendorffperfused rat hearts (8). The present data confirm that autoregulation is intrinsic to the heart.

The relationship between cardiac work and the oxygen supply is likely to be of critical importance to this. Oxygen extraction is nearly maximal from the circulating blood in the heart, therefore an increased oxygen supply can be achieved only by increasing coronary flow via coronary vasodilatation (5). Exercise-induced tachycardia results in an increased coronary blood flow due to decreased coronary vascular resistance (5), as shown in several species (2530). Increased production of adenosine and other endogenous vasodilator substances, such as bradykinin, atrial natriuretic peptide or nitric oxide have been reported to contribute to this $(5$, 31-33). The rate-dependence of flow that we observed is in agreement with published data (8), and in this respect validates the method used. 
4.1.2. Mechanism by which ventricular irregularity increases coronary flow in the physiological heart rate range in Langendorff perfused hearts

Beat-to-beat variability of the ventricular cycle length is referred to 'heart rate variability' when measured in sinus rhythm in vivo, and heart rate variability is a biomarker of vagal nerve activity in vivo (34). However, the present experiments were performed in isolated, Langendorff perfused hearts (denervated, in the absence of the rest of the animal). Thus, the effect of cycle length variability on coronary flow was not mediated by parasympathetic activity or any effect of the autonomic nervous system.

The results of the second set of experiments showed that the positive effect of ventricular irregularity on coronary flow in the physiological heart rate range was mediated via changes in left ventricular pressure, presumably via ventricular compression of coronary arterioles. In regular rhythm, left ventricular (and intramural) pressures overcame perfusion pressure in systole, reversing the direction of aortic flow for a short period during systole in each beat. In contrast, a single premature beat produced at least two beats, in which left ventricular pressure remained below the perfusion pressure in systole. This suggests that the positive effect of ventricular irregularity on coronary flow is specific to the isolated heart preparation perfused at constant pressure, and it does not necessary involve any change in workinduced autoregulation.

\subsubsection{Balance between left ventricular load and ejection in Langendorff perfused hearts}

Real-time aortic flow, perfusion pressure and left ventricular pressure signals imply that left ventricle ejected during systole in regular rhythm in the present experiments with Langendorff perfused guinea pig hearts. Real-time aortic flow values showed that there was an apparent balance between left ventricular filling and ejection in each beat in regular rhythm. A crucial question emerges: how does the perfusion fluid enter into the left ventricle in Langendorff perfused hearts? According to the original description by Langendorff, when perfusion solution flows retrogradely to the aorta, the aortic valve is closed $(2,35)$, therefore the left ventricle should not be filled with perfusion solution. One explanation is that the aortic valve was incompetent in the present experiments. Technical failure (e.g., valve damage during insertion of the aortic cannula) is unlikely as special care was taken during preparation to avoid this. Also, the dicrotic notch in the aortic pressure signal (Figure 4) implies that aortic valves were functioning normally. A more plausible explanation is that that the intact (not disrupted) aortic valve does not close properly in isolated hearts perfused with constant pressure, and there is a considerable leakage of perfusion fluid into the left ventricle in normal circumstances (36). 
Wiggers used dog, cat and rabbit hearts on a constant pressure perfusion system, and measured the outflow from the filled right atrium, and the fluid entering the left ventricle was drained by a cannula pushed through the left ventricle wall (36). Massive leakage was recorded from the left ventricle in most of the experiments when the perfusion cannula was in the aorta. However when the perfusion cannula was inserted directly into the coronaries, the leakage decreased significantly, but a small amount of fluid still entered into the left ventricle through the Thebesian vessels (36). Our results accord with Wiggers' data and show that the Langendroff perfused guinea pig heart is loaded just like the isolated, perfused dog, cat and rabbit hearts (36). Furthermore, results of the present investigation imply that ventricular irregularity disturbs the balance between load and ejection, which consequently affects left ventricular and intramural pressures, and thus, influences coronary flow in isolated hearts.

\subsubsection{Pharmacological relevance}

The proarrhythmic liability of almost every newly developed drug has to be evaluated (37). Isolated Langendorff-perfused hearts are frequently used for proarrhythmia investigations $(18,19)$, and ancillary readout such as coronary flow helps form an integrated risk assessment (38). The present results indicate that drug-induced arrhythmias may affect coronary flow independently of heart rate in isolated hearts, meaning any observed flow changes will include a component that is entirely independent of direct coronary vascular actions and rate-dependent vascular tone changes. Also, a drug that inhibits arrhythmias may be expected to evoke rateindependent decremental effects on coronary flow that could be falsely interpreted as a coronary vasoconstrictor adverse drug action. These possibilities may now be anticipated and accounted for when coronary flow data are interpreted in any such drug study in which rhythm alterations occur.

\subsection{Comparing arrhythmia results obtained according to the old and the updated}

\section{Lambeth Conventions - validation of arrhythmia definitions by using arrhythmia data}

\section{from Langendorff perfused hearts}

Present results show that arrhythmia analysis according to LC II qualitatively changed conclusions about pharmacological effects of flecainide. The change in tachyarrhythmia definitions also affected well documented pathophysiological effects, i.e. the preventive effect of the high $\mathrm{K}^{+}$concentration against ischaemic VF disappeared when the VF definition of LC II was applied. The reason behind these changes was that VF definition of LC II identified 'de novo' VF episodes not identified according to LC I, which is supported by the data showing 
that application of the VF definition of LC II increased VF incidence and reduced VF onset time. Importantly, a very strong inter-observer agreement was found among the two independent investigators, when VF incidence obtained according to LC I was tested. Applying VF definition of LC II resulted in less inter-observer agreement on VF incidence.

\subsubsection{Arrhythmia shift from VT to VF category enlarges the VF class of LC II and markedly}

\section{increases sensitivity of VF detection}

VF definition of LC II diagnosed VF in all hearts, in which VF was identified according to LC I. Moreover, there were many other hearts, in which VF was detected only according to LC II and VF was not found according to LC I. Also, applying VF definition of LC II did not increase VF onset time in any of the hearts, however reduced this variable in the majority of the hearts. These results show that the VF class of LC II incorporated and enlarged the VF class defined by LC I. The reason behind this is that VF definition of LC II not only identified VF episodes detected by LC I, but also identified 'de novo' VF episodes not detected according to LC I. Our results clearly show that the enlarged VF category of LC II substantially increased the sensitivity of VF detection.

Comparing tachyarrhythmia definitions of LC I and LC II (see Table 1) explains the mechanism of detection of 'de novo' VF episodes according to LC II. While arrhythmias containing 4 or more consecutive ventricular premature beats with non-progressive variation in peak-peak interval, height and intrinsic shape should be classified as VTs according to LC I, the same arrhythmias are now shifted to the VF category of LC II (Figure 15) (see also Table 1 for the arrhythmia definitions of LC I and LC II)(12, 13). These polymorphic VTs of LC I form the 'de novo' VF episodes detected by LC II. This 'arrhythmia shift' of LC II is also indicated by the data showing that applying arrhythmia definitions of LC II reduced the number of VT episodes and simultaneously increased the number of VF episodes. 

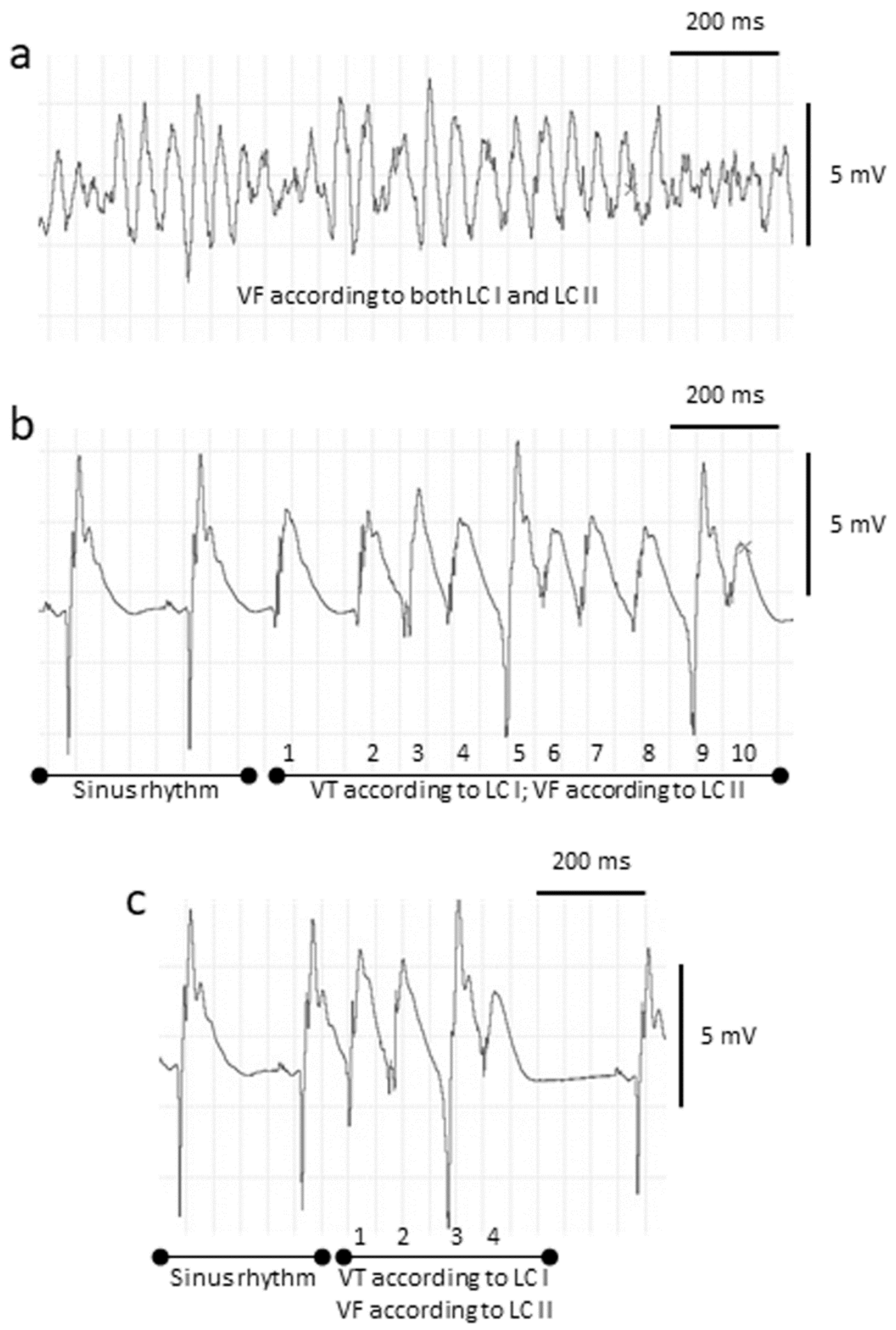

Figure 15. Incompatibility between the classes of the ventricular fibrillation of the Lambeth Conventions I (LC I) and Lambeth Conventions II (LC II). Part a: ventricular tachyarrhythmia diagnosed as ventricular fibrillation (VF) according to both LC I and LC II. Part b: a ten-beat tachyarrhythmia episode of 'de novo' VF as this ventricular tachyarrhythmia is diagnosed as ventricular tachycardia (VT) according to LC I, while the same episode is diagnosed as VF according to LC II. LC II emphasised the importance of 'diastolic pause'. According to LC II, "if an arrhythmia is interrupted by an identifiable pause or quiescence (a 'diastolic pause', or a flat isoelectric line) that is longer than the prevailing sinus rate (or idioventricular rate if the 
$A V$ node is blocked) then it makes more sense to classify each arrhythmia present before and after the pause as separate events"(13). Based on the above recommendation of LC II, there is no relevant diastolic pause between the ventricular complexes of this tachyarrhythmia, thus this arrhythmia should be regarded as one single run of arrhythmia. Also note that the arrhythmia consists of sections with four consecutive beats (beats 3-4-5-6 and beats 7-8-9-10), in which neither intrinsic shape, nor peek-peek interval, nor height of the ventricular complexes change progressively, thus each of these four-beat arrhythmia sections should be classified as VF according to LC II (See Table 1 for the arrhythmia definitions). Convention 14 of LC II states that "When rhythms segue from one to another without an identifiable interruption or quiescence, or vary between simultaneously recorded leads, then the arrhythmia should be classified as the most serious manifestation within segue or lead."(13). As this arrhythmia in part $b$ should be regarded as one single run of arrhythmia and VF is the most serious arrhythmia manifestation within segue, the whole arrhythmia should be classified as VF according to LC II. On the other hand, QRS deflections can be distinguished from one another and the rate can be measured, thus the same arrhythmia should be classified as VT according to LC I. Part c: a four-beat tachyarrhythmia episode of 'de novo' $\mathrm{VF}$ as this ventricular tachyarrhythmia is diagnosed as VT according to LC I, while the same episode is diagnosed as VF according to LC II. The ECG examples were recorded in three isolated, Langendorff perfused rat hearts subjected to regional ischaemia for $30 \mathrm{~min}$.

Interestingly, Observer A found that when arrhythmia definitions of LC II were applied, the cumulative number of VT and VF episodes was greater than the respective value obtained according to LC I. The phenomenon of 'arrhythmia fragmentation' is most likely the reason behind this result. LC II introduced the concept of the importance of 'diastolic pause'. According to LC II, "if an arrhythmia is interrupted by an identifiable pause or quiescence (a 'diastolic pause', or a flat isoelectric line) that is longer than the prevailing sinus rate (or idioventricular rate if the $A V$ node is blocked) then it makes more sense to classify each arrhythmia present before and after the pause as separate events" (13). This concept enables an arrhythmia episode to be fragmented into many shorter arrhythmia episodes. Consequently, the number of VT and VF episodes measured according to LC I can be affected by 'arrhythmia fragmentation' when the same arrhythmias are evaluated according to LC II. However, the increase in VF incidence and the reduction in VF onset time caused by LC II can only be explained by the 'arrhythmia shift' phenomenon, and 'arrhythmia fragmentation' could not contribute to these results. It can be concluded that the 'arrhythmia shift' caused by LC II is responsible for the only moderate intra-observer agreement on VF incidence values obtained according to LC I and LC II. 
4.2.2. Applying the VF definition of LC II may change the conclusions of pharmacological investigations

Applying VF definition of LC II qualitatively changed conclusions about the pharmacological effect of flecainide by questioning the significant antifibrillatory effect of the drug seen when the VF definition of LC I was applied. The effectiveness of flecainide against ischaemic VF is not well characterized as results of previous investigations are confounding (39-43). Thus, it is not possible to decide which VF definition can provide data that describes the real face of this drug. However, our results provide the first clear cut evidence that by changing the definition of VF the analysis of the same ECG recordings may yield a completely different conclusion.

The analysis of the VF onset times in the first set of experiments showed that application of the VF definition of LC II increases VF detection in all groups, not only in the flecainidetreated group, but even in the quinidine- and lidocaine-treated groups and also in the control group. These results imply that the change in VF definition may retrospectively change the results of previous pharmacological studies analysed according to LC I. As mentioned earlier, over 1000 studies have already based their conclusions on data obtained according to the definitions of LC I. Changing the definition of VF may invalidate the conclusions of many of these previous investigations, and also make it impossible to compare future results that will have been obtained according to LC II to previous results obtained according to LC I.

\subsubsection{Effect of $K^{+}$on VF incidence, application of the VF definition of LC II may invalidate} well-accepted physiological and pathophysiological concepts and studies based on these concepts

Hyperkalaemia can occur in various experimental and clinical settings (44-46). The physiological and pathophysiological effects of potassium in the development of arrhythmias have been intensively studied in earlier investigations. Arrhythmias following coronary occlusion may depend on factors such as serum potassium, i.e. higher potassium concentrations reduce the incidence of ischaemic VF in rats (47). Hyperkalaemia is antiarrhythmic during myocardial ischemia and infarction in man; serum potassium concentrations obtained on admission to hospital were inversely related to the incidence of ventricular fibrillation (48). An association between low serum potassium concentrations and ventricular arrhythmias has also been observed by a number of investigators (49) and an increased frequency of ventricular fibrillation in patients with low serum potassium concentrations was also demonstrated(50). Furthermore, in the GISSI-2 trial, the incidence of VF among patients with a serum potassium 
$<3.6 \mathrm{mEq} / \mathrm{L}$ was nearly twice that seen in patients with a higher serum potassium levels (51). Only one of the above mentioned investigations (52) used the VF definition of LC I, still there was a good agreement between these studies on their results about the effect of potassium on ischaemic VF. Thus, it can be concluded that the effect of potassium on ischaemic VF is independent of the VF definition of LC I. However, based on the results of the above mentions investigations and the results of several other previous experimental and clinical investigations, it is now widely accepted that the incidence of ischaemic VF inversely correlates with the potassium concentration $(12,13,52,53)$.

When we analysed the effect of $\mathrm{K}^{+}$concentration on VF incidence in the control groups and applied the VF definition of LC I, results were in a good agreement with the above mentioned experimental and clinical data. This analysis showed that VF incidence was significantly lower in the control group perfused with Krebs solution containing $5 \mathrm{mM} \mathrm{K}^{+}$than that in the control group perfused with $3 \mathrm{mM} \mathrm{K}^{+}$. However when we applied the VF definition of LC II, the VF incidence was very high in the control groups irrespective of the $\mathrm{K}^{+}$ concentration of the Krebs solution. This suggests that increased sensitivity of VF detection according to LC II coincided with markedly reduced specificity. Consequently, application of VF definition of LC II substantially changed the conclusion about the physiological and pathophysiological effects of $\mathrm{K}^{+}$concentration on VF development. Present results provide the first clear-cut evidence that applying VF definition of LC II may change the conclusion not only in pharmacological but also in physiological and pathophysiological arrhythmia investigations.

Importantly, application of the VF definition of LC II would totally invalidate the whole proarrhythmia investigation with $5 \mathrm{mM} \mathrm{K}^{+}$in the perfusate in 3rd and 4th set of experiments in the original investigation $(15,16)$, as increased VF incidence in the control hearts perfused with $5 \mathrm{mM} \mathrm{K}^{+}$does not provide scope for examining profibrillatory effects of the test drugs. Present results show that application of the VF definition of LC II may invalidate a widely known and accepted concept of pathophysiology of arrhythmia development, and also invalidate earlier investigations based on this concept. This is a warning sign, which suggests that the tachyarrhythmia (VT and VF) definitions of LC II should be abandoned or at least amended in order to provide greater compatibility with the results of earlier investigations.

\subsubsection{Excellent inter-observer agreement with VF definition of LC I, smaller inter-observer} agreement with VF definition of LC II 
Present investigation provides a strong evidence that VF results are well reproducible, and the inter-observer agreement is high, when the VF definition of LC I is applied. This suggests that the VF definition of LC I is clear and it is not necessary to be changed in order to provide greater objectivity during arrhythmia evaluation, which was one of the main aims of introducing the new VF definition of LC II (13). This aim was clearly not fulfilled by the new VF definition of LC II, as applying VF definition of LC II resulted in less inter-observer agreement on VF incidence. Furthermore, VT definition of LC II did not remarkably improve inter-observer agreement on VT incidence. These suggest that applying VT and VF definitions of LC II does not improve compatibility of the results of various research groups and investigators, which further emphasizes the need for an amendment of the VT and VF definitions of LC II. 


\section{CONCLUSIONS}

According our results from the first study there is a positive, linear correlation between mean ventricular rate and coronary flow in isolated Langendorff perfused guinea pig hearts, but variability of the ventricular cycle length influences this correlation independently of mean ventricular rate. When cycle length variability increases, the slope of the regression line gets steeper. Thus, in the physiological heart rate range, an autonomic-independent increase in coronary flow occurs in guinea pig hearts, which is mediated by cycle length irregularity via producing beats that have left ventricular pressure reduced below perfusion pressure. Changes in ventricular rhythm will therefore affect coronary flow independently of heart rate in isolated hearts perfused at constant pressure, and this fact should be noted in drug studies on arrhythmias performed in Langendorff perfused hearts.

The second investigation provides the first clear-cut evidence that arrhythmia results obtained by applying ventricular tachyarrhythmia definitions of LC I and LC II are not compatible in Langendorff perfused rat hearts. This calls for further research in order to test the validity of the arrhythmia definitions of LC II in other species and models, too. Nevertheless, present results indicate that applying VF definition of LC II may change the conclusion of previous pharmacological, physiological and pathophysiological arrhythmia investigations. The reason behind this is that VF class of LC II incorporated and enlarged the VF class defined by LC I. The enlarged VF category of LC II substantially increases the sensitivity of VF detection, but it coincides with markedly reduced specificity. Furthermore, present investigation provides an evidence that VF incidence results are well reproducible, and the inter-observer agreement is high, when the VF definition of LC I is applied. However, applying VF definition of LC II may reduce inter-observer agreement on VF incidence. Thus, it is concluded that VT and VF definitions of LC II should be amended in order to allow better interobserver agreement and greater compatibility with the results of earlier investigations obtained by applying arrhythmia definitions of LC I. However, any modified definitions should be subjected to research in order to test their validity.

The present thesis shows that despite the Langendroff perfused heart model is old and simple, it is still a powerful tool for heart research, and arrhythmia analysis in Langendorff perfused hearts significantly contributed to clarification and characterisation of the physiology of the model and also, to validation of arrhythmia definitions. 


\section{REFERENCES}

1. Skrzypiec-Spring M, Grotthus B, Szelag A, Schulz R. Isolated heart perfusion according to Langendorff---still viable in the new millennium. J Pharmacol Toxicol Methods. 2007;55(2):113-26.

2. Bell RM, Mocanu MM, Yellon DM. Retrograde heart perfusion: the Langendorff technique of isolated heart perfusion. J Mol Cell Cardiol. 2011;50(6):940-50.

3. Colatsky T, Fermini B, Gintant G, Pierson JB, Sager P, Sekino Y, et al. The Comprehensive in Vitro Proarrhythmia Assay (CiPA) initiative - Update on progress. J Pharmacol Toxicol Methods. 2016;81:15-20.

4. Yokokawa M, Kim HM, Good E, Crawford T, Chugh A, Pelosi F, Jr., et al. Impact of QRS duration of frequent premature ventricular complexes on the development of cardiomyopathy. Heart Rhythm. 2012;9(9):1460-4.

5. Duncker DJ, Bache RJ, Merkus D. Regulation of coronary resistance vessel tone in response to exercise. J Mol Cell Cardiol. 2012;52(4):802-13.

6. Kingma JG, Jr., Rouleau JR. Coronary vasoregulation in health and disease. Can J Cardiol. 2007;23 Suppl B:9B-14B.

7. Cooper MW. Postextrasystolic potentiation. Do we really know what it means and how to use it? Circulation. 1993;88(6):2962-71.

8. Bernier M, Curtis MJ, Hearse DJ. Ischemia-induced and reperfusion-induced arrhythmias: importance of heart rate. Am J Physiol. 1989;256(1 Pt 2):H21-31.

9. Duncker DJ, Merkus D. Exercise hyperaemia in the heart: the search for the dilator mechanism. J Physiol. 2007;583(Pt 3):847-54.

10. Priori SG, Blomstrom-Lundqvist C, Mazzanti A, Blom N, Borggrefe M, Camm J, et al. 2015 ESC Guidelines for the management of patients with ventricular arrhythmias and the prevention of sudden cardiac death: The Task Force for the Management of Patients with Ventricular Arrhythmias and the Prevention of Sudden Cardiac Death of the European Society of Cardiology (ESC)Endorsed by: Association for European Paediatric and Congenital Cardiology (AEPC). Europace. 2015;17(11):1601-87.

11. Al-Khatib SM, Stevenson WG, Ackerman MJ, Bryant WJ, Callans DJ, Curtis AB, et al. 2017 AHA/ACC/HRS Guideline for Management of Patients With Ventricular Arrhythmias and the Prevention of Sudden Cardiac Death. Circulation. 2018;138(13):e272e391.

12. Walker MJ, Curtis MJ, Hearse DJ, Campbell RW, Janse MJ, Yellon DM, et al. The Lambeth Conventions: guidelines for the study of arrhythmias in ischaemia infarction, and reperfusion. Cardiovasc Res. 1988;22(7):447-55.

13. Curtis MJ, Hancox JC, Farkas A, Wainwright CL, Stables CL, Saint DA, et al. The Lambeth Conventions (II): guidelines for the study of animal and human ventricular and supraventricular arrhythmias. Pharmacol Ther. 2013;139(2):213-48.

14. Curtis MJ. Characterisation, utilisation and clinical relevance of isolated perfused heart models of ischaemia-induced ventricular fibrillation. Cardiovasc Res. 1998;39(1):194215.

15. Farkas A, Curtis MJ. Limited antifibrillatory effectiveness of clinically relevant concentrations of class I antiarrhythmics in isolated perfused rat hearts. J Cardiovasc Pharmacol. 2002;39(3):412-24.

16. Farkas A, Curtis MJ. Does QT widening in the Langendorff-perfused rat heart represent the effect of repolarization delay or conduction slowing? J Cardiovasc Pharmacol. 2003;42(5):612-21. 
17. Farkas A, Qureshi A, Curtis MJ. Inadequate ischaemia-selectivity limits the antiarrhythmic efficacy of mibefradil during regional ischaemia and reperfusion in the rat isolated perfused heart. Br J Pharmacol. 1999;128(1):41-50.

18. Farkas AS, Acsai K, Toth A, Dezsi L, Orosz S, Forster T, et al. Importance of extracardiac alpha1-adrenoceptor stimulation in assisting dofetilide to induce torsade de pointes in rabbit hearts. Eur J Pharmacol. 2006;537(1-3):118-25.

19. Farkas AS, Makra P, Csik N, Orosz S, Shattock MJ, Fulop F, et al. The role of the $\mathrm{Na}+/ \mathrm{Ca} 2+$ exchanger, $\mathrm{I}(\mathrm{Na})$ and $\mathrm{I}(\mathrm{CaL})$ in the genesis of dofetilide-induced torsades de pointes in isolated, AV-blocked rabbit hearts. Br J Pharmacol. 2009;156(6):920-32.

20. Farkas AS, Rudas L, Makra P, Csik N, Lepran I, Forster T, et al. Biomarkers and endogenous determinants of dofetilide-induced torsades de pointes in alpha(1) -adrenoceptorstimulated, anaesthetized rabbits. Br J Pharmacol. 2010;161(7):1477-95.

21. Vincze D, Farkas AS, Rudas L, Makra P, Csik N, Lepran I, et al. Relevance of anaesthesia for dofetilide-induced torsades de pointes in alpha1-adrenoceptor-stimulated rabbits. Br J Pharmacol. 2008;153(1):75-89.

22. Sarusi A, Rarosi F, Szucs M, Csik N, Farkas AS, Papp JG, et al. Absolute beat-to-beat variability and instability parameters of ECG intervals: biomarkers for predicting ischaemiainduced ventricular fibrillation. Br J Pharmacol. 2014;171(7):1772-82.

23. Pedersen PA, Kristensen FB. [The Danish Medical Statistics and Danish practical research]. Ugeskr Laeger. 1990;152(12):828-9.

24. Rouleau JR, Simard D, Rodrigue N, Blouin A, Kingma JG, Jr. Myocardial blood flow after chronic cardiac decentralization in anesthetized dogs: effects of ACE-inhibition. Auton Neurosci. 2002;97(1):12-8.

25. Duncker DJ, Stubenitsky R, Verdouw PD. Autonomic control of vasomotion in the porcine coronary circulation during treadmill exercise: evidence for feed-forward betaadrenergic control. Circ Res. 1998;82(12):1312-22.

26. Khouri EM, Gregg DE, Rayford CR. Effect of exercise on cardiac output, left coronary flow and myocardial metabolism in the unanesthetized dog. Circ Res. 1965;17(5):427-37.

27. Laughlin MH, Klabunde RE, Delp MD, Armstrong RB. Effects of dipyridamole on muscle blood flow in exercising miniature swine. Am J Physiol. 1989;257(5 Pt 2):H1507-15. 28. Manohar M. Left ventricular oxygen extraction during submaximal and maximal exertion in ponies. J Physiol. 1988;404:547-56.

29. Vatner SF, Higgins CB, Millard RW, Franklin D. Role of the spleen in the peripheral vascular response to severe exercise in untethered dogs. Cardiovasc Res. 1974;8(2):276-82.

30. von Engelhardt W. Cardiovascular effects of exercise and training in horses. Adv Vet Sci Comp Med. 1977;21:173-205.

31. Achs MJ, Garfinkel D, Opie LH. Computer simulation of metabolism of glucoseperfused rat heart in a work-jump. Am J Physiol. 1982;243(3):R389-99.

32. Berne RM. Cardiac nucleotides in hypoxia: possible role in regulation of coronary blood flow. Am J Physiol. 1963;204:317-22.

33. Vial C, Owen P, Opie LH, Posel D. Significance of release of adenosine triphosphate and adenosine induced by hypoxia or adrenaline in perfused rat heart. J Mol Cell Cardiol. 1987;19(2):187-97.

34. Koizumi K, Terui N, Kollai M. Effect of cardiac vagal and sympathetic nerve activity on heart rate in rhythmic fluctuations. J Auton Nerv Syst. 1985;12(2-3):251-9.

35. O L. Untersuchungen am überlebenden Säugethierherzen. Pflüger's Archiv für die gesamte Physiologie des Menschen und der Tiere. 1895;61:41.

36. CJ W. The innervation of the coronary vessel. Am J Physiol. 1909(24):14. 
37. Farkas AS, Nattel S. Minimizing repolarization-related proarrhythmic risk in drug development and clinical practice. Drugs. 2010;70(5):573-603.

38. Pugsley MK, Authier S, Curtis MJ. Principles of safety pharmacology. Br J Pharmacol. 2008;154(7):1382-99.

39. Gout B, Nichols AJ, Feuerstein GZ, Bril A. Antifibrillatory effects of BRL-32872 in anesthetized Yucatan minipigs with regional myocardial ischemia. J Cardiovasc Pharmacol. 1995;26(4):636-44.

40. Lederman SN, Wenger TL, Bolster DE, Strauss HC. Effects of flecainide on occlusion and reperfusion arrhythmias in dogs. J Cardiovasc Pharmacol. 1989;13(4):541-6.

41. Winslow E, Campbell JK, Barron E, Marshall RJ, Muir AW. Effects of Org 7797 on early, late and inducible arrhythmias following coronary artery occlusion in rats and dogs. $\mathrm{Br}$ J Pharmacol. 1991;104(4):853-8.

42. Barrett TD, Hayes ES, Walker MJ. Lack of selectivity for ventricular and ischaemic tissue limits the antiarrhythmic actions of lidocaine, quinidine and flecainide against ischaemia-induced arrhythmias. Eur J Pharmacol. 1995;285(3):229-38.

43. Barrett TD, Hayes ES, Yong SL, Zolotoy AB, Abraham S, Walker MJ. Ischaemia selectivity confers efficacy for suppression of ischaemia-induced arrhythmias in rats. Eur J Pharmacol. 2000;398(3):365-74.

44. Deska P, Nowicki M. Short-term changes of serum potassium concentration induced by physical exercise in patient with arterial hypertension treated with angiotensin-converting enzyme inhibitor alone or in combination with statin. J Physiol Pharmacol. 2017;68(1):133-8. 45. Wojcik B, Knapp M, Gorski J. Non-ischemic heart preconditioning. J Physiol Pharmacol. 2018;69(2).

46. Wojcik B, Miklosz A, Zabielski P, Chabowski A, Gorski J. Effect of tachycardia on mRNA andf protein expression of the principal components of the lipolytic system in the rat's heart ventricles. J Physiol Pharmacol. 2017;68(5):731-6.

47. Chang KW, TJ OL, Price M. Evaluation of unilateral referrals on neonatal hearing screening. J Med Screen. 2009;16(1):17-21.

48. Nordrehaug JE, von der Lippe G. Hypokalaemia and ventricular fibrillation in acute myocardial infarction. Br Heart J. 1983;50(6):525-9.

49. Papp H, Sarusi A, Farkas AS, Takacs H, Kui P, Vincze D, et al. Hyperventilation assists proarrhythmia development during delayed repolarization in clofilium-treated, anaesthetized, mechanically ventilated rabbits. J Physiol Pharmacol. 2016;67(5):731-7. 50. Solomon RJ. Ventricular arrhythmias in patients with myocardial infarction and ischaemia. The role of serum potassium. Drugs. 1986;31 Suppl 4:112-20.

51. Volpi A, Cavalli A, Santoro L, Negri E. Incidence and prognosis of early primary ventricular fibrillation in acute myocardial infarction--results of the Gruppo Italiano per lo Studio della Sopravvivenza nell'Infarto Miocardico (GISSI-2) database. Am J Cardiol. 1998;82(3):265-71.

52. Curtis MJ, Hearse DJ. Ischaemia-induced and reperfusion-induced arrhythmias differ in their sensitivity to potassium: implications for mechanisms of initiation and maintenance of ventricular fibrillation. J Mol Cell Cardiol. 1989;21(1):21-40.

53. Cohn JN, Kowey PR, Whelton PK, Prisant LM. New guidelines for potassium replacement in clinical practice: a contemporary review by the National Council on Potassium in Clinical Practice. Arch Intern Med. 2000;160(16):2429-36. 


\section{ACKNOLEDGEMENTS}

I would like to thank Professor Tamás Forster, former director of the $2^{\text {nd }}$ Department of Internal Medicine and Cardiology Centre for his support during my PhD studies.

I also want to thank Professor András Varró, former director of the Department of Pharmacology and Pharmacotherapy, for his support and for providing me the facilities in his Department for the experimental work during my PhD studies.

I am also thankful for my supervisor, Dr. András Farkas for his endless help and guidance during my studies and work.

I wish to thank Professor Julius Gy. Papp from the Department of Pharmacology and Pharmacotherapy for his wise pieces of advice and his support I have enjoyed during these years.

I would like to acknowledge my dear friend and colleague, Dr. Péter Kui for his much needed help and support during my studies and experiments.

I would like to express my gratitude for my family especially, my friends and all of my colleagues at the $2^{\text {nd }}$ Department of Internal Medicine and Cardiology Centre and at the Department of Pharmacology and Pharmacotherapy for all their help and support. Without them, this work would not have been completed. 


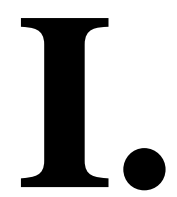




\title{
Ventricular cycle length irregularity affects the correlation between ventricular rate and coronary flow in isolated, Langendorff perfused guinea pig hearts
}

\author{
Hedvig Takács ${ }^{\text {a }}$, Péter Kui ${ }^{\text {b }}$, Attila S. Farkas a , Annamária Sarusi ${ }^{\text {b }}$, Tamás Forster ${ }^{\text {a }}$, Julius Gy. Papp ${ }^{\text {b,c }}$, \\ András Varró ${ }^{\mathrm{b}}$, Michael J. Curtis ${ }^{\mathrm{d}}$, Michael J. Shattock ${ }^{\mathrm{d}}$, András Farkas ${ }^{\mathrm{a}, *}$ \\ a Second Department of Medicine and Cardiology Centre, Faculty of Medicine, University of Szeged, Szeged, Hungary \\ ${ }^{\mathrm{b}}$ Department of Pharmacology and Pharmacotherapy, Faculty of Medicine, University of Szeged, Szeged, Hungary \\ c MTA-SZTE Research Group for Cardiovascular Pharmacology, Hungarian Academy of Sciences, Hungary \\ d King's College, London, UK
}

\section{A R T I C L E I N F O}

Available online 9 October 2015

\section{Keywords:}

Perfusion pressure

Coronary flow

Guinea pig

Isolated hearts

Langendorff perfusion

Left ventricular pressure

Methods

Ventricular rate

Ventricular cycle length variability

Irregular rhythm

\begin{abstract}
A B S T R A C T
Introduction: Heart rate affects coronary flow, but the mechanism is complex. The relationship between rhythm and flow is unclear, especially in experimental settings used for determining drug actions. The present study examined whether ventricular irregularity influences coronary flow independently of heart rate.

Methods: Guinea pig hearts were perfused (Langendorff mode) at constant pressure. Hypokalemic Krebs solution facilitated spontaneous development of arrhythmias. The ECG, left ventricular and perfusion pressures were recorded, and the coronary flow was measured. Beat-to-beat ventricular cycle length variability was quantified. Hearts were retrospectively allocated to arbitrary 'Low' or 'High' RR variability groups.

Results: A positive linear correlation was found between mean ventricular rate and coronary flow. The slope of the regression line was significantly greater in the 'High' versus 'Low' RR variability group, with greater coronary flow values in the 'High' RR variability group in the physiological heart rate range. During regular rhythm, left ventricular pressure exceeded perfusion pressure and prevented coronary perfusion at peak systole. However, ventricular irregularity significantly increased the number of beats in which left ventricular pressure remained below perfusion pressure, facilitating coronary perfusion.

Discussion: In isolated hearts, cycle length irregularity increases the slope of the positive linear correlation between mean ventricular rate and coronary flow via producing beats in which left ventricular pressure remains below perfusion pressure. This means that changes in rhythm have the capacity to influence coronary flow independently of heart rate in isolated hearts perfused at constant pressure, which should be noted in drug studies on arrhythmias performed in Langendorff hearts.
\end{abstract}

(c) 2015 Elsevier Inc. All rights reserved.

\section{Introduction}

Understanding the relationship between ventricular rhythm and coronary flow autoregulation in experimental preparations such as the Langendorff is important for several reasons.

Many patients live with irregular ventricular rate caused by either frequent ventricular or atrial arrhythmias. Irregular ventricular rate may be harmful in the long term, e.g. it is well documented that frequent ventricular premature beats (VPB) can lead to development of

Abbreviations: APB, Atrial premature beat; PESP, Post-extrasystolic potentiation; RMSSD, Root mean square of the successive difference of the RR intervals; VPB Ventricular premature beat.

* Corresponding author at: 2nd Department of Medicine and Cardiology Centre, Faculty of Medicine, University of Szeged, Semmelweis utca 6., H-6725 Szeged, Hungary.

E-mail address: farkas.andras@med.u-szeged.hu (A. Farkas). cardiomyopathy (Yokokawa et al., 2012). However, it is not known whether an effect of irregular ventricular rhythm on coronary flow may contribute to the harmful effects of irregular ventricular rhythm.

In in vivo conditions coronary flow is regulated by a combination of i) intramural pressure in coronary arteries caused by wall stress during the cardiac cycle, ii) the autonomic nervous system, iii) and workinduced autoregulation via local metabolites (Duncker, Bache, \& Merkus, 2012; Kingma \& Rouleau, 2007). Additionally, irregular rhythm affects the work of the myocardium independently of rate and load (Cooper, 1993). This implies that irregular ventricular rhythm may have an independent effect on coronary flow via modifying the workinduced autoregulation of the coronary arteries. However, this has never been examined, and data about the well-known positive correlation between ventricular rate and coronary flow have been obtained from hearts free of arrhythmias (Bernier, Curtis, \& Hearse, 1989; Duncker \& Merkus, 2007). As it is not known how work-induced 
autoregulation of coronary arteries is affected by ventricular irregularity, the approach of the present study was to determine the effect of beat-to-beat variability of ventricular cycle length on coronary flow in isolated, Langendorff-perfused guinea pig hearts.

Caval veins do not fill the right atrium in Langendorff-perfused heart, and thus when the hydrostatic pressure of the perfusion column is constant, hearts can be studied with coronary arteries perfused under constant pressure (Curtis, 1998). Also, Langendorff-perfused hearts are normally denervated. Thus, under these conditions coronary flow is regulated independently of perfusion pressure and autonomic nervous system, and only intramural pressure during the cardiac cycle and workdependent autoregulation determine coronary resistance. As ventricular irregularity was found to significantly affect coronary flow in the present investigation, the mechanism was examined in a second set of experiments performed in Langendorff perfused guinea pig hearts.

\section{Methods}

\subsection{Animals and general experimental methods}

Female guinea pigs ( $n=87$ in the first set of experiments and $n=33$ in the second), weighing 300-400 g were used. The animal-handling protocol was in accordance with the Guidance of the Operation on the Animals (Scientific Procedures) Act 1986 and the European Community guidelines for the use of experimental animals.

The method of Langendorff perfusion we used has been described in detail (Farkas \& Curtis, 2002, 2003; Farkas, Qureshi, \& Curtis, 1999). Briefly, animals were anesthetized with pentobarbital (60 mg/kg i.p.) mixed with $1000 \mathrm{IU}$ sodium heparin to prevent blood clot formation in the coronary vasculature. Sodium heparin (500 IU) was additionally administered i.v. Hearts were excised and placed immediately into ice-cold modified Krebs-solution containing: $118.5 \mathrm{mM} \mathrm{NaCl}, 25.0 \mathrm{mM}$ $\mathrm{NaHCO}_{3}, 0.5 \mathrm{mM} \mathrm{MgSO}_{4}, 1.2 \mathrm{mM} \mathrm{NaH}_{2} \mathrm{PO}_{4}, 1.8 \mathrm{mM} \mathrm{CaCl}, 3.0 \mathrm{mM} \mathrm{KCl}$, and $11.1 \mathrm{mM}$ glucose. Langendorff perfusion was initiated with solution delivered at $37^{\circ} \mathrm{C}$ and $\mathrm{pH} 7.4$ at constant perfusion pressure $(60 \mathrm{mmHg})$. In the first set of experiments, a unipolar electrogram (ECG) was recorded by implanting one stainless-steel wire electrode into the middle of the anterior wall of the left ventricle with a second connected to the aorta. In the second set of experiments, volume conducted ECG was recorded by submerging the ventricles of the hearts in modified Krebs solution at $37^{\circ} \mathrm{C}$.

\subsection{Measurement of coronary flow}

Coronary flow was measured by timed collection of coronary effluent. At the end of the experiment atria were removed from the hearts and ventricles were weighed. Coronary flow values are shown in $\mathrm{ml} / \mathrm{min} / \mathrm{g}$.

\subsection{ECG analysis, measurement of the RR intervals and calculation of variability of the ventricular cycle length in the first set of experiments}

The ECG was recorded and analyzed by LabChart7 (ADInstruments Ltd., Oxford, UK). In the guinea pig Langendorff preparation, noncomplex arrhythmias (mostly VPBs, atrial premature beats [APBs], and sinus arrhythmia) occur frequently during the initial period after mounting the heart, especially if Krebs solution contains a low concentration of $\mathrm{K}^{+}(3.0 \mathrm{mM})$ and a high concentration of $\mathrm{Ca}^{2+}(1.8 \mathrm{mM})$. However, these arrhythmias spontaneously resolve within 20-30 min. The reason for these baseline arrhythmias is not known; it is a particular feature of guinea pig hearts and the same perfusion method does not cause arrhythmias in rat and rabbit hearts (Farkas \& Curtis, 2002; Farkas et al., 2006). These ventricular and atrial arrhythmic beats were defined according to the Lambeth Conventions II (Curtis et al., 2013) and manually counted in the last $30 \mathrm{~s}$ of the 30 -min-long control perfusion period. The percent frequency of arrhythmic beats (defined as
APBs, VPBs or individual QRT complexes in a run of a salvo or tachycardia) was calculated in the sampling period as the number of ventricular arrhythmic beats divided by the total number of beats times 100 .

The RR intervals were measured irrespective of rhythm even during arrhythmias in the 30-s-long sampling period. The mean ventricular rate was calculated as the total number of ventricular complexes (arrhythmic or not) times 2 . The beat-to-beat variability of the RR intervals was quantified by the root mean square of the successive differences of the RR intervals (RMSSD) as described previously (Farkas et al., 2009): taking the successive differences of the RR intervals $\left(\Delta d_{j}=d_{j+1}-\right.$ $d_{j} ; 0 \leq j \leq N-2$, where $d_{j}$ represents the RR interval durations and $N$ is the total number of intervals) and calculating $R M S S D=\sqrt{E\left([\Delta \mathrm{d}]^{2}\right)}$, where $E$ denotes the mean value. RMSSD of the RR interval during sinus rhythm in vivo is a parameter widely used for quantifying heart rate variability as a biomarker of parasympathetic activity (Farkas et al., 2010; Vincze et al., 2008), but in the Langendorff preparation it quantifies only the irregularity of the cycle length since parasympathetic tone is absent.

These hearts were divided into two groups based on RMSSD value as the 'Low' RR variability group (RMSSD $<3 \mathrm{~ms} ; n=50$ hearts), and the 'High' RR variability group (RMSSD $>3 \mathrm{~ms} ; n=37$ hearts) (Fig. 1 ). The beat-to-beat variability of the cycle length (RMSSD), the percent frequency of the arrhythmic beats, the mean ventricular rate and the coronary flow were compared between the 'Low' and 'High' RR variability groups. Note (with respect to justification of animal usage) that all hearts were entered into a separate, unrelated experimental protocol 30 min after the start of perfusion.

\subsection{Measurement of the duration of perfused and non-perfused intervals in every cardiac cycle}

It was found in the first set of experiments that cycle length irregularity increased the slope of the linear correlation between mean ventricular rate and coronary flow. Myocardium in vivo is perfused during diastole and is not perfused when intramural capillaries are compressed by intramural pressure during systole. In order to examine whether elevated RR interval variability increased the ratio of durations of perfused intervals to non-perfused intervals in the Langendorff preparation, a second set of experiments was performed with 33 hearts perfused identically for 60 min with the same modified Krebs solution but with hearts submerged in Krebs solution in order to record volume conducted ECG. Left ventricular pressure was recorded via a thin medical needle stuck through the apex of the heart, and attached to a plastic cannula filled with saline. Perfusion pressure (aortic pressure) was recorded via a side arm at the bottom of the perfusion column where perfusion cannula was connected to the aorta. "Real-time" aortic flow was measured by an ultrasonic flow meter (T106 Animal Research Flowmeter, Transonic Systems Inc. Ithaca, NY, U.S.A) implanted to the bottom of the perfusion column, closely above the aortic stump. Krebs solution was continuously pumped to the reservoir by a pump (Peri-star Pro, World Precision Instruments, Sarasota, Florida, U.S.A.) during the experiment and a built-in overflow system kept the height of the column at a pre-set, constant level, thus the hydrostatic pressure of the perfusion column was constant $(65 \mathrm{mmHg}$ ). Volume-conducted ECG, real-time aortic flow, aortic and left ventricular pressures were recorded continuously by using National Instruments data acquisition hardware (PC card, National Instruments, Austin, TX., U.S.A.) and SPEL Advanced Haemosys software (version 3.26, Experimetria Ltd. and Logirex Software Laboratory, Budapest, Hungary).

RR interval variability (RMSSD of the RR interval), mean ventricular rate and mean aortic flow were determined in every 30-s-long interval during the 60 -min-long perfusion period in each heart. A strong, positive, linear correlation was found between the measured coronary flow and the calculated mean aortic flow values $(y=1.0166 x+$ 0.5482 where $y$ is the mean aortic flow value $(\mathrm{ml} / \mathrm{min} / \mathrm{g})$ and $x$ is the measured coronary flow value $(\mathrm{ml} / \mathrm{min} / \mathrm{g})$; regression coefficient: $R=$ 


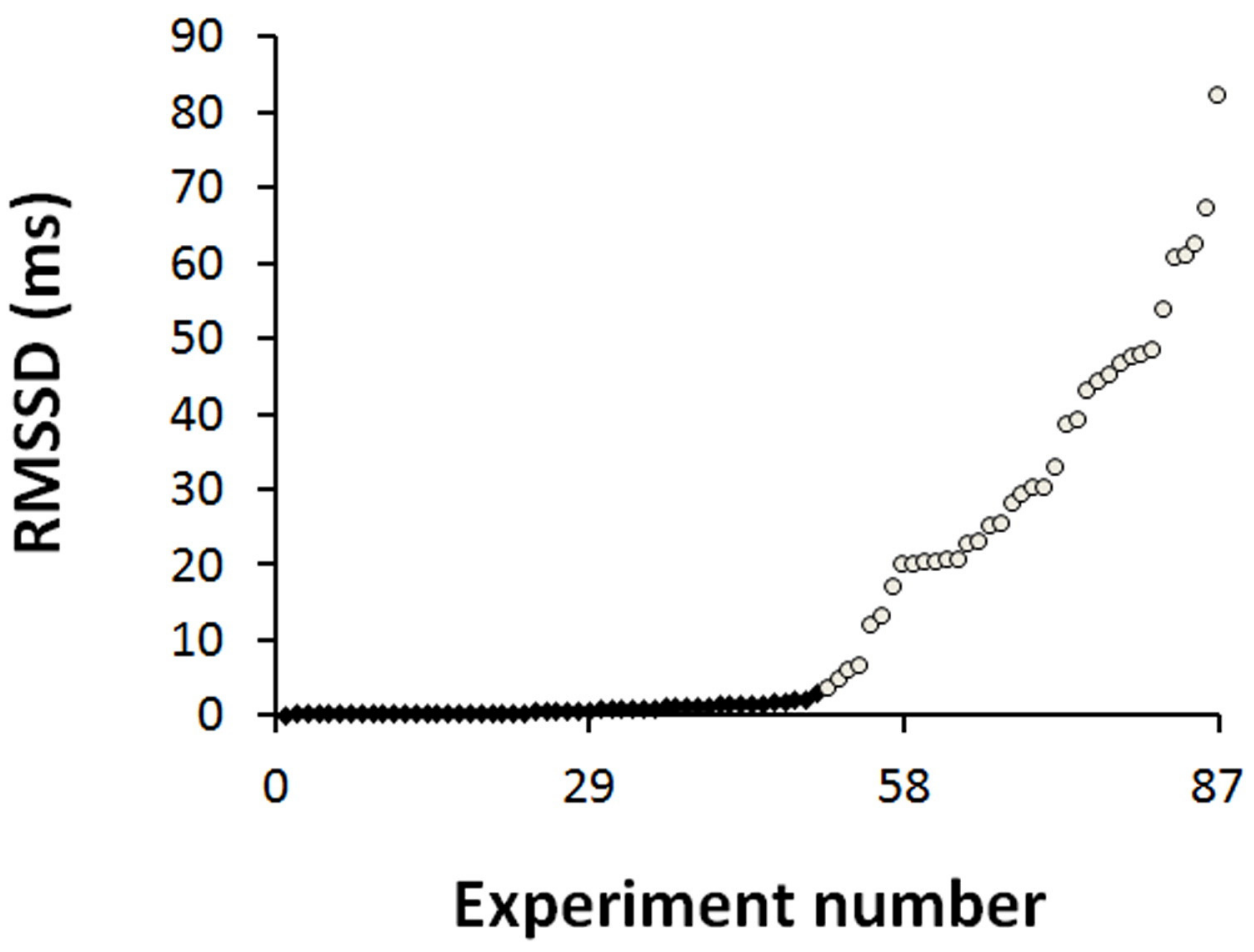

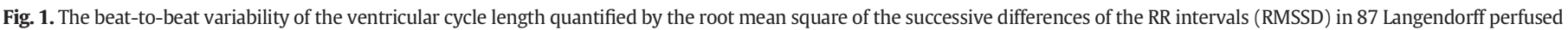

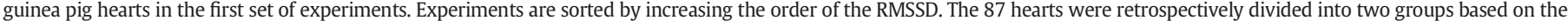

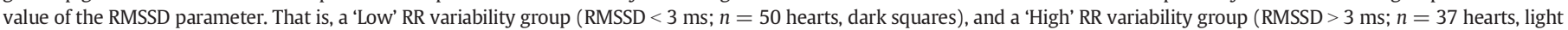
circles) were arbitrarily defined.

0.966; $P<0.0001$, linear regression). In 15 hearts, the ventricular rate did not match the mean ventricular rate found in the first set of experiments, thus these hearts were excluded from the analysis. In each of the remaining 18 hearts, there was at least one 30-s-long interval in which mean ventricular rate matched the mean ventricular rate measured in the first set of experiments. One of these 30-s-long intervals was chosen for further analysis in each heart. These 18 hearts thus replicated the RR interval variability and mean ventricular rate of the 'Low' and 'High' RR variability groups in the first set of experiments, with 9 hearts allocated into a 'Low' RR interval variability group (RMSSD $<3 \mathrm{~ms}$ ), and the other 9 to a 'High' RR interval variability group (RMSSD $>3 \mathrm{~ms}$ ).

An analysis of the durations of perfused and non-perfused intervals in the 18 hearts was performed in a blinded manner. The number of beats in which left ventricular pressure did not exceed perfusion pressure during systole, and the duration of the non-perfused intervals during each systole were determined. The non-perfused interval (i.e. the interval where intramural pressure prevents coronary perfusion) was defined as the interval in which left ventricular pressure was greater than perfusion pressure during systole. Perfused interval (i.e. the interval where intramural pressure allows coronary perfusion) was defined as the interval in which left ventricular pressure was lower than perfusion pressure. The ratio of the perfused to non-perfused intervals was calculated by dividing the cumulative duration of the perfused intervals by the cumulative duration of the non-perfused intervals in the 30-s-long sampling interval at the time point of the measurement.

\subsection{Statistical analysis}

Continuous data were expressed as mean \pm standard deviation (SD) and the 'Low' and 'High' RR variability groups were compared using the non-parametric Mann-Whitney $U$ test. Within group comparison of continuous data was performed using the non-parametric Wilcoxon test. The coronary flow values were plotted against the mean ventricular rate values. The correlation between mean ventricular rate and coronary flow was examined with analysis of covariance, in which variables of coronary flow (as the continuous dependent variable), mean ventricular rate (as the continuous predictor variable, i.e. covariate) and group ('Low' vs. 'High' RR variability group, as the categorical grouping variable) were tested. $P<0.05$ was taken as indicative of a statistically significant difference between values.

\section{Results}

\subsection{First set of experiments, irregular rhythm affects coronary flow}

First, the beat-to-beat variability of the ventricular cycle length was tested as an independent variable influencing coronary flow. RMSSD values were significantly greater in the 'High' RR variability group than in the 'Low' RR variability group (Fig. 2a). This reflects the intensity of arrhythmias; the percent frequency of arrhythmic beats was significantly greater in the 'High' RR variability group versus the 'Low' RR variability group (percent frequency in the 'High' RR variability group: mean 14 , median 2.7, range $0-53$ vs. percent frequency in the 'Low' RR variability group: mean: 0 ; median 0 range: $0-0.8$; $P<0.05$ ). RR interval variability was inevitably increased by spontaneously occurring arrhythmias during the observed 30-s-long period in the 'High' RR variability group: VPBs occurred in 25 hearts (an average of 21 beats, median 5 beats, range $1-58$ beats), however VPBs with short coupling interval (R-on-T VPBs) occurred very rarely (in 6 hearts, an average of 3 beats, median 2.5 beats, range $1-5$ beats); APB-s occurred in 9 hearts (an average of 12 beats, median 4 beats, range 2-47 beats); increased variability of RR intervals was caused only by sinus arrhythmia in 10 hearts. Hearts in the 'Low' RR variability group remained in regular sinus rhythm.

Importantly, the mean ventricular rate did not differ significantly between the two groups (Fig. 2b), whereas the coronary flow was significantly greater in the 'High' RR variability group (Fig. 2c). 


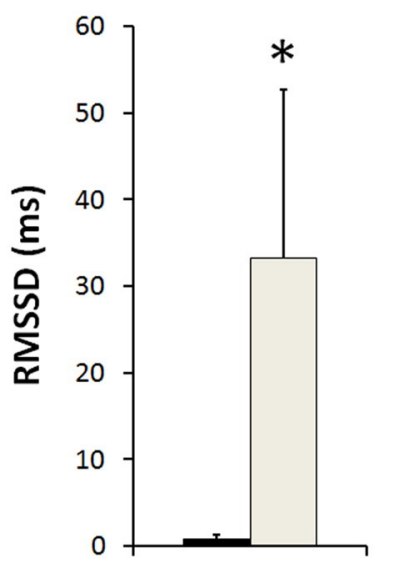

b.

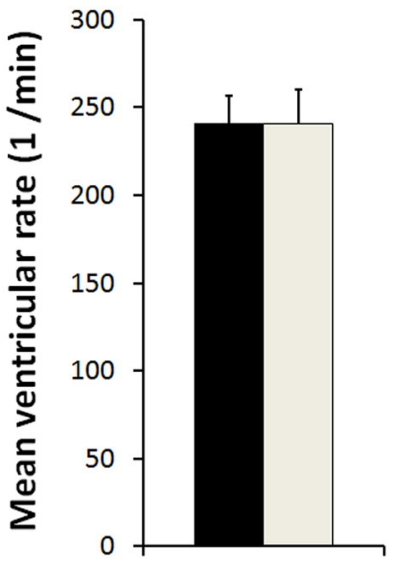

C.

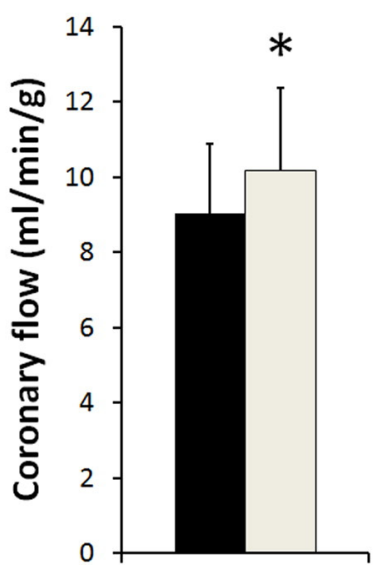

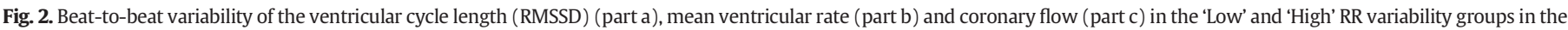

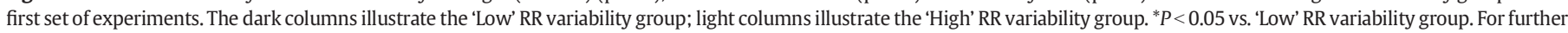
details, see Fig. 1.

The relationship between mean ventricular rate and coronary flow was tested in a further analysis. A significant positive linear correlation was found between these variables in the 'Low' RR variability group, but with a low correlation coefficient (Fig. 3). A similar relationship was seen in the 'High' RR variability group (Fig. 3). However, the slope of the regression line was significantly greater in the 'High' versus 'Low' RR variability group, with consequentially greater coronary flow values in the 'High' RR variability group in the physiological heart rate range for guinea pig (210-280 1/min) (Fig. 3).

\subsection{Second set of experiments; duration of perfused and non-perfused intervals}

The RMSSD, mean ventricular rate and coronary flow values measured in the first set of experiments were reproduced in the second set of experiments. Accordingly, the beat-to-beat variability of the ventricular cycle length was significantly greater in the 'High' RR variability group than in the 'Low' RR variability group (RMSSD: $29.4 \pm 16.2 \mathrm{~ms}$ vs. $1.0 \pm 0.5 \mathrm{~ms}$, respectively; $P<0.05)$. Mean ventricular rate did not differ between the 'High' and 'Low' RR variability groups ( $240 \pm 7$ vs. $236 \pm 41 / \mathrm{min}$, respectively). Mean aortic flow (which strongly correlated with coronary flow; see Section 2) was significantly greater in the 'High' RR variability group than in the 'Low' RR variability group $(8.9 \pm 1.8 \mathrm{ml} / \mathrm{min} / \mathrm{g}$ vs. $6.8 \pm 1.1 \mathrm{ml} / \mathrm{min} / \mathrm{g}$, respectively; $P<0.05)$.

The analysis of the real time aortic flow signal, the perfusion pressure and the left ventricular pressure in each cardiac cycle revealed that the left ventricular pressure exceeded the perfusion pressure in most beats in hearts with 'Low' RR variability, associated with a transient reversal of the direction of aortic flow (Fig. 4). The average (per beat) duration of the non-perfused interval (in which left ventricular pressure exceeded perfusion pressure) did not differ significantly between the 'High' and 'Low' RR variability groups ( $37 \pm 10 \mathrm{~ms}$ vs. $38 \pm 8 \mathrm{~ms}$, respectively). However, cumulative number of beats that lacked non-perfused interval was greater in the 'High' RR variability group than in the 'Low' RR variability group (Fig. 5a). Consequently, the cumulative duration of the perfused intervals was significantly

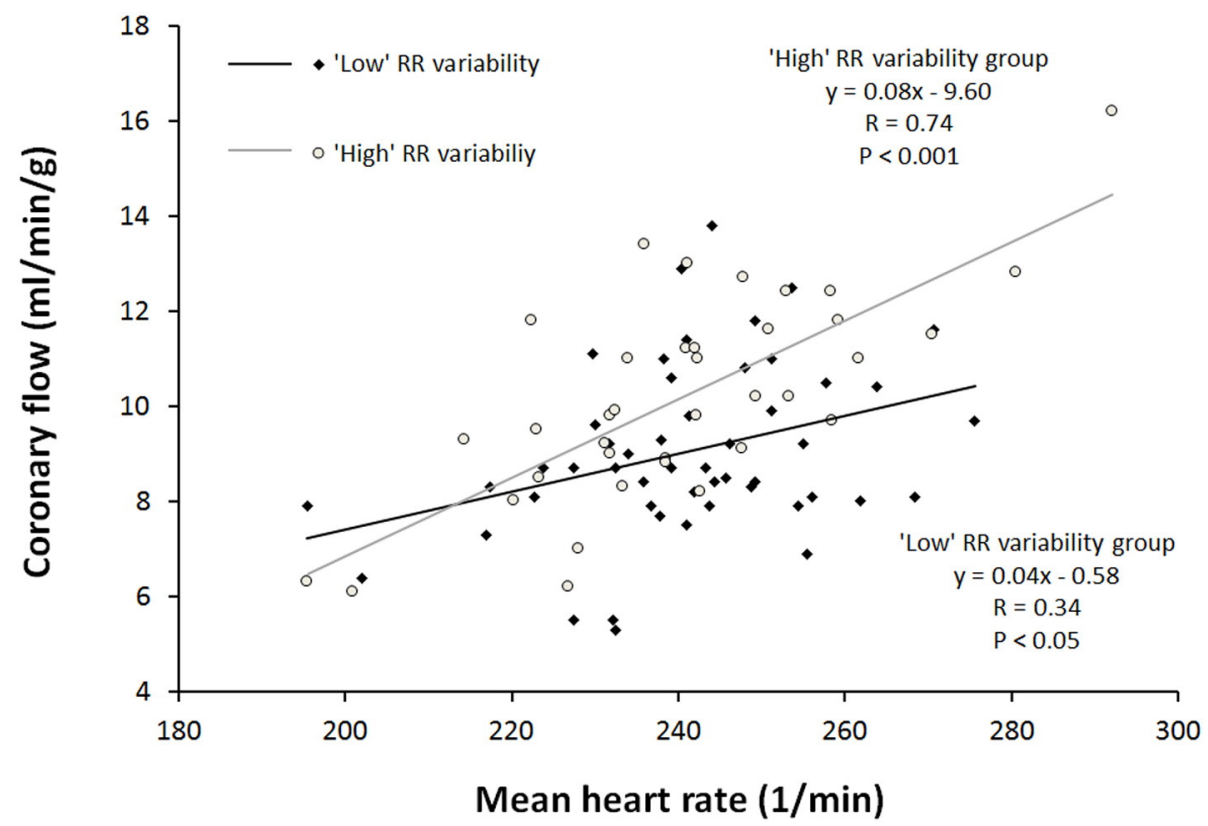

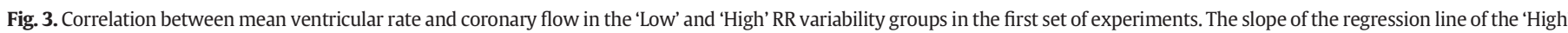
RR variability group is significantly greater than that of the 'Low' RR variability group ( 0.08 vs. 0.04 , respectively; ${ }^{*} P<0.05$, analysis of covariance). 


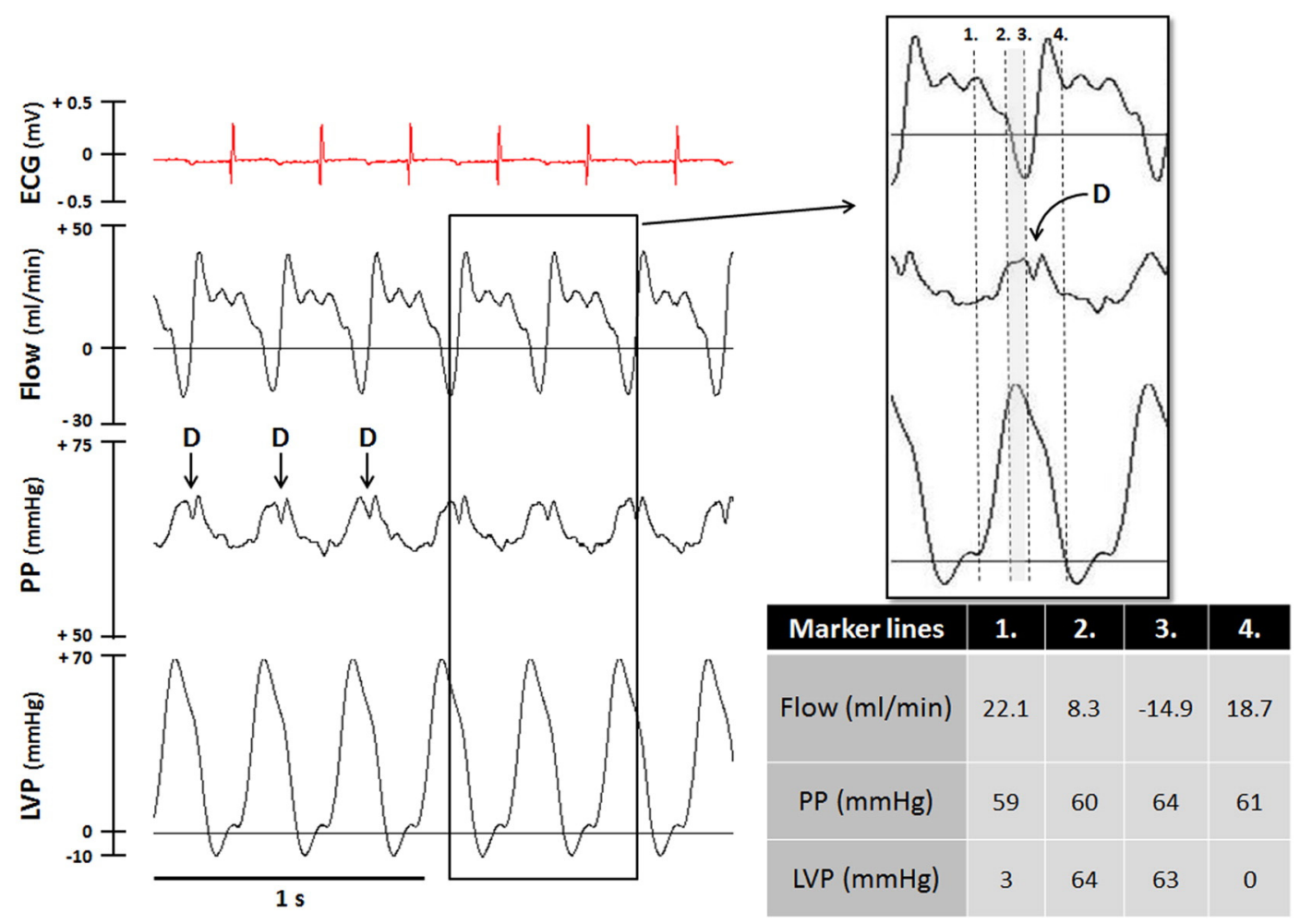

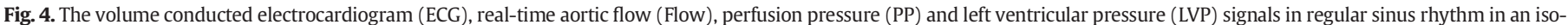

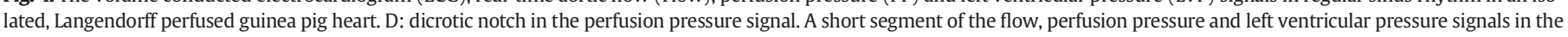

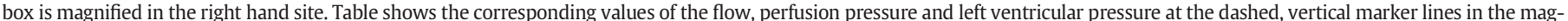

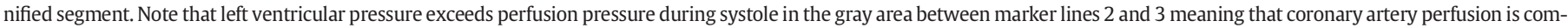

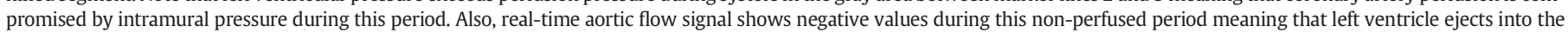

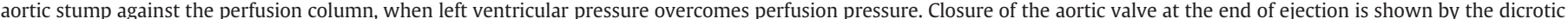

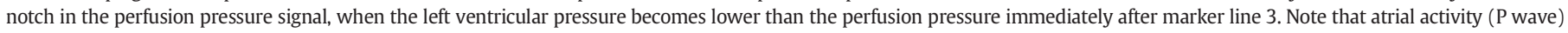
is not recognizable in the volume conducted ECG signal as only ventricles were submerged.

greater in the 'High' RR variability group than in the 'Low' RR variability group (Fig. 6a). Thus, ventricular irregularity significantly increased the ratio of the perfused to non-perfused intervals (Fig. 6c). Usually, the beats that lacked non-perfused interval were atrial or ventricular premature beats and beats that follow 'post-extrasystolic potentiation' (PESP) beats (Fig. 7).

\section{Discussion}

There is a positive linear correlation between mean ventricular rate and coronary flow in Langendorff perfused guinea pig hearts. However, the slope of the regression line is steeper when beat-to-beat variability of ventricular cycle length increases, which results in an increase in coronary flow in the physiological heart rate range independently of mean RR interval.

In the present investigation with Langendorff perfused guinea pig hearts, left ventricular pressure at peak systole exceeded perfusion pressure. The positive effect of increased beat-to-beat variability of the cycle length on coronary flow resulted from the preponderance of beats in which left ventricular pressure did not exceed perfusion pressure during systole, facilitating coronary perfusion. a.

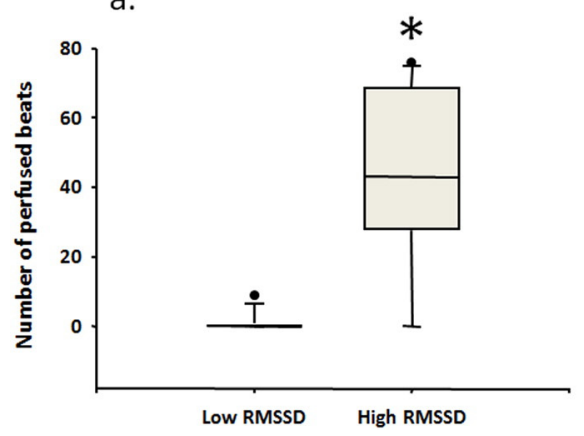

b.

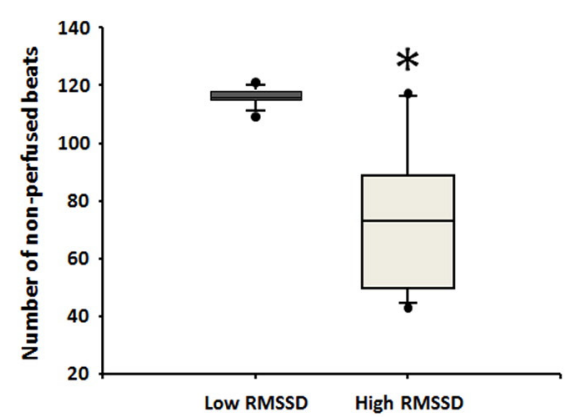

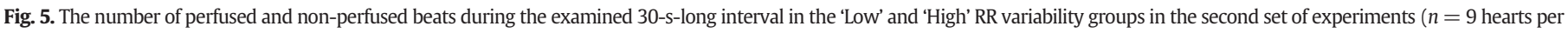

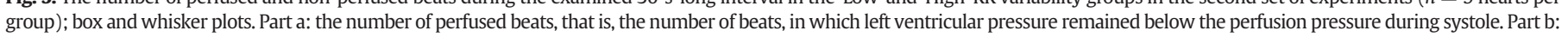
the number of non-perfused beats, that is, the number of beats, in which left ventricular pressure exceeded perfusion pressure during systole. ${ }^{*} P<0.05$ vs. 'Low' RR variability group. 
a.

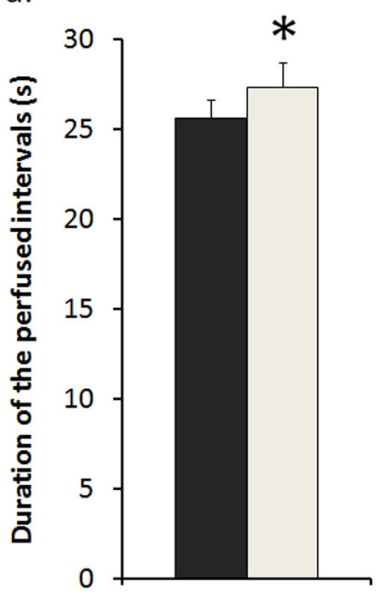

b.

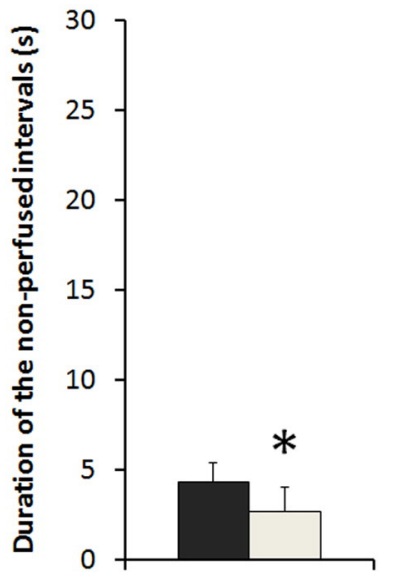

C.

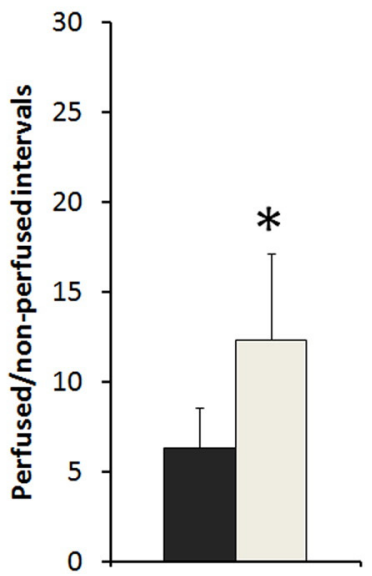

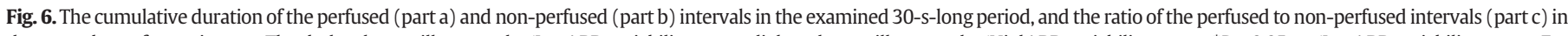

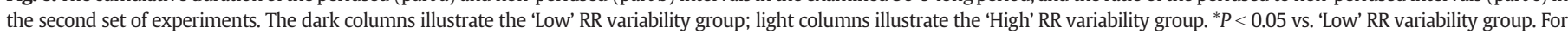
further details, see Fig. 5.

4.1. Positive, linear correlation between the ventricular rate and coronary flow

The linear correlation between mean ventricular rate and coronary flow reflects the established relationship between coronary blood flow in vivo and myocardial oxygen and nutrient demand (Duncker et al., 2012; Kingma \& Rouleau, 2007). One interesting implication from the present study is confirmation that autoregulation is largely autonomic-independent (Langendorff hearts are denervated). In vivo, the autonomic nervous system may influence autoregulation (Kingma \& Rouleau, 2007), but the relationship is preserved in autonomically denervated dogs (Kingma \& Rouleau, 2007; Rouleau, Simard, Rodrigue,

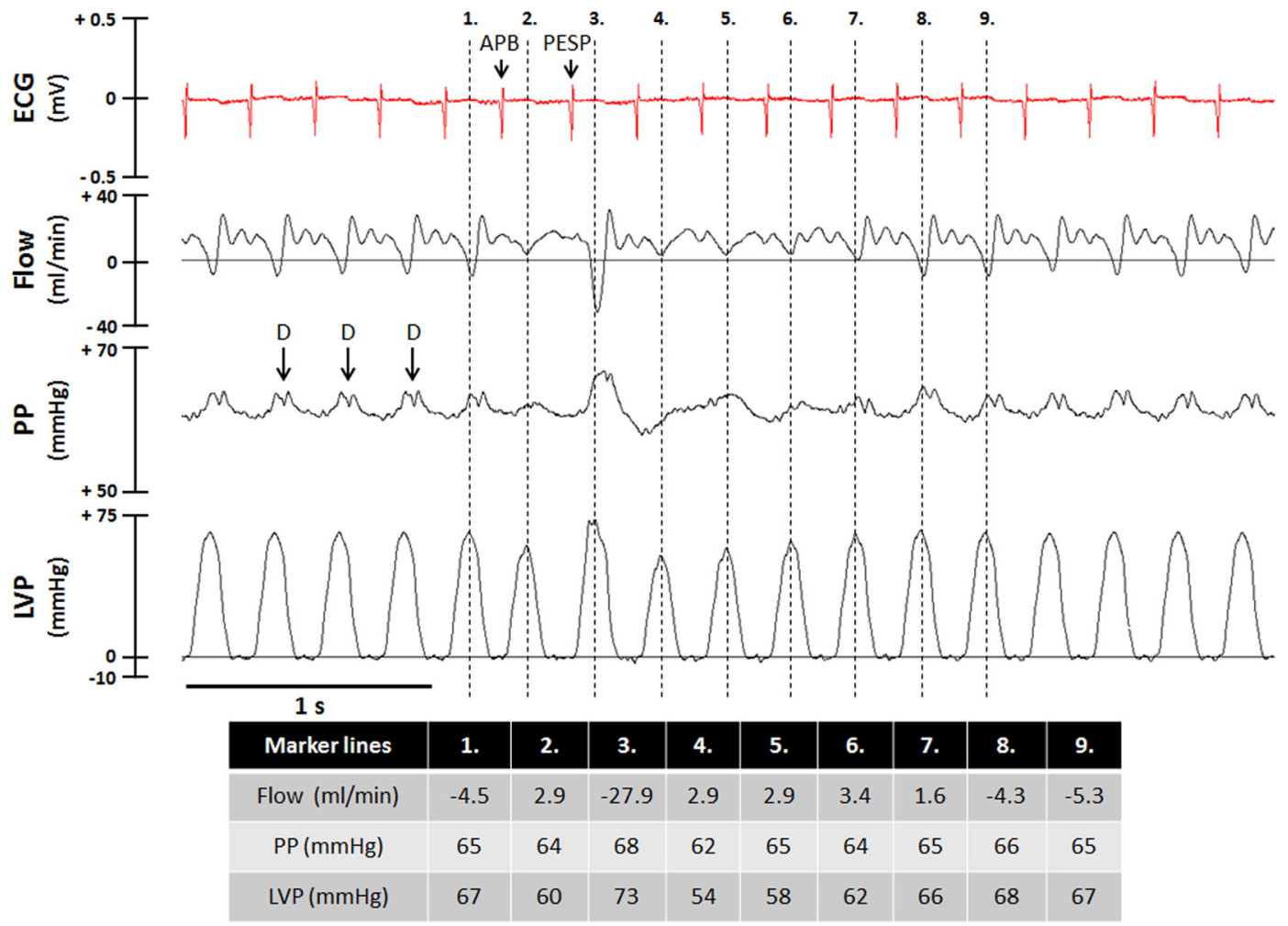

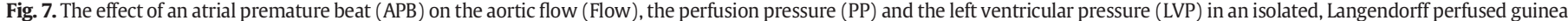

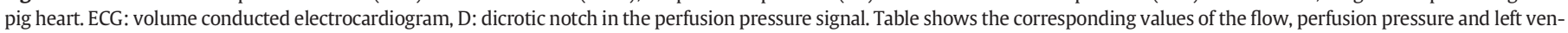

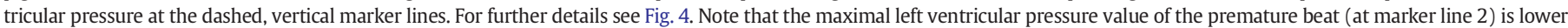

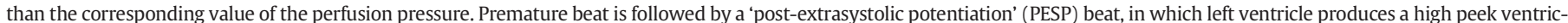

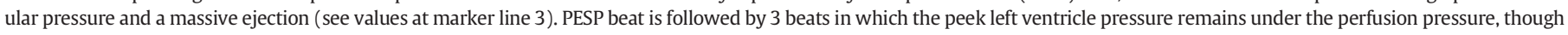

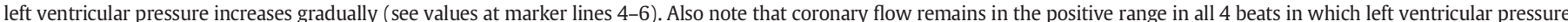

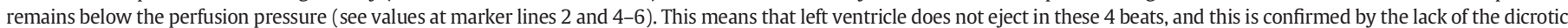

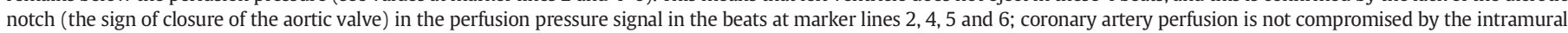
pressure during the entire duration of these 4 beats. 
Blouin, \& Kingma, 2002), and in isolated, denervated, Langendorffperfused rat hearts (Bernier et al., 1989). The present data confirm that autoregulation is intrinsic to the heart.

The relationship between cardiac work and the oxygen supply is likely to be of critical importance to this. Oxygen extraction is nearly maximal from the circulating blood in the heart, therefore an increased oxygen supply can be achieved only by increasing coronary flow via coronary vasodilatation (Duncker et al., 2012). Exercise-induced tachycardia results in an increased coronary blood flow due to decreased coronary vascular resistance (Duncker et al., 2012), as shown in several species (Duncker, Stubenitsky, \& Verdouw, 1998; Khouri, Gregg, \& Rayford, 1965; Laughlin, Klabunde, Delp, \& Armstrong, 1989; Manohar, 1988; Vatner, Higgins, Millard, \& Franklin, 1974; von Engelhardt, 1977). Increased production of adenosine and other endogenous vasodilator substances, such as bradykinin, atrial natriuretic peptide or nitric oxide have been reported to contribute to this (Achs, Garfinkel, \& Opie, 1982; Berne, 1963; Duncker et al., 2012; Vial, Owen, Opie, \& Posel, 1987). The ratedependence of flow that we observed is in agreement with published data (Bernier et al., 1989), and in this respect validates the method used.

4.2. Mechanism by which ventricular irregularity increases coronary flow in the physiological heart rate range in Langendorff perfused hearts

Beat-to-beat variability of the ventricular cycle length is referred to 'heart rate variability' when measured in sinus rhythm in vivo, and heart rate variability is a biomarker of vagal nerve activity in vivo (Koizumi, Terui, \& Kollai, 1985). However, the present experiments were performed in isolated, Langendorff perfused hearts (denervated, in the absence of the rest of the animal). Thus, the effect of cycle length variability on coronary flow was not mediated by parasympathetic activity or any effect of the autonomic nervous system.

The results of the second set of experiments showed that the positive effect of ventricular irregularity on coronary flow in the physiological heart rate range was mediated via changes in left ventricular pressure, presumably via ventricular compression of coronary arterioles. In regular rhythm, left ventricular (and intramural) pressures overcame perfusion pressure in systole, reversing the direction of aortic flow for a short period during systole in each beat. In contrast, a single premature beat produced at least two beats, in which the left ventricular pressure remained below the perfusion pressure in systole. This suggests that the positive effect of ventricular irregularity on coronary flow is specific to the isolated heart preparation perfused at constant pressure, and it does not necessarily involve any change in workinduced autoregulation.

\subsection{Balance between left ventricular load and ejection in Langendorff perfused hearts}

Real-time aortic flow, perfusion pressure and left ventricular pressure signals imply that left ventricle ejected during systole in regular rhythm in the present experiments with Langendorff perfused guinea pig hearts. Real-time aortic flow values showed that there was an apparent balance between left ventricular filling and ejection in each beat in regular rhythm. A crucial question emerges: How does the perfusion fluid enter into the left ventricle in Langendorff perfused hearts? According to the original description by Langendorff, when perfusion solution flows retrogradely to the aorta, the aortic valve is closed (Bell, Mocanu, \& Yellon, 2011; Langendorf, 1895), therefore the left ventricle should not be filled with perfusion solution. One explanation is that the aortic valve was incompetent in the present experiments. Technical failure (e.g. valve damage during insertion of the aortic cannula) is unlikely as special care was taken during preparation to avoid this. Also, the dicrotic notch in the aortic pressure signal (Fig. 4) implies that aortic valves were functioning normally. A more plausible explanation is that the intact (not disrupted) aortic valve does not close properly in isolated hearts perfused with constant pressure, and there is a considerable leakage of perfusion fluid into the left ventricle in normal circumstances (Wiggers, 1909). Wiggers used dog, cat and rabbit hearts on a constant pressure perfusion system, and measured the outflow from the filled right atrium, and the fluid entering the left ventricle was drained by a cannula pushed through the left ventricle wall (Wiggers, 1909). Massive leakage was recorded from the left ventricle in most of the experiments when the perfusion cannula was in the aorta. However when the perfusion cannula was inserted directly into the coronaries, the leakage decreased significantly, but a small amount of fluid still entered into the left ventricle through the Thebesian vessels (Wiggers, 1909). Our results accord with Wiggers' data and show that the Langendorff perfused guinea pig heart is loaded just like the isolated, perfused dog, cat and rabbit hearts (Wiggers, 1909). Furthermore, results of the present investigation imply that ventricular irregularity disturbs the balance between load and ejection, which consequently affects left ventricular and intramural pressures, and thus, influences coronary flow in isolated hearts.

\subsection{Pharmacological relevance}

The proarrhythmic liability of almost every newly developed drug has to be evaluated (Farkas \& Nattel, 2010). Isolated Langendorffperfused hearts are frequently used for proarrhythmia investigations (Farkas et al., 2006; Farkas et al., 2009), and ancillary readout such as coronary flow helps form an integrated risk assessment (Pugsley, Authier, \& Curtis, 2008). The present results indicate that druginduced arrhythmias may affect coronary flow independently of heart rate in isolated hearts, meaning any observed flow changes will include a component that is entirely independent of direct coronary vascular actions and rate-dependent vascular tone changes. Also, a drug that inhibits arrhythmias may be expected to evoke rate-independent decremental effects on coronary flow that could be falsely interpreted as a coronary vasoconstrictor adverse drug action. These possibilities may now be anticipated and accounted for when coronary flow data are interpreted in any such drug study in which rhythm alterations occur.

\section{Conclusions}

There is a positive, linear correlation between mean ventricular rate and coronary flow in isolated Langendorff perfused guinea pig hearts, but the variability of the ventricular cycle length influences this correlation independently of mean ventricular rate. When cycle length variability increases, the slope of the regression line gets steeper. Thus, in the physiological heart rate range, an autonomic-independent increase in coronary flow occurs in guinea pig hearts, which is mediated by cycle length irregularity via producing beats that have left ventricular pressure reduced below perfusion pressure. Changes in ventricular rhythm will therefore affect coronary flow independently of heart rate in isolated hearts perfused at constant pressure, and this fact should be noted in drug studies on arrhythmias performed in Langendorff perfused hearts.

\section{Acknowledgments}

This work was supported by Heptagon Foundation, Burton Fund, and Hungarian Scientific Research Fund [OTKA PD 105882] and National Development Agency and European Regional Fund [TÁMOP-4.2.2-08/12008-0013, TÁMOP-4.2.1/B-09/1/KONV-2010-0005]. Dr. Attila S. Farkas is a holder of János Bolyai fellowship (BO/00794/12) of the Hungarian Academy of Sciences.

\section{References}

Achs, M. J., Garfinkel, D., \& Opie, L. H. (1982). Computer simulation of metabolism of glucose-perfused rat heart in a work-jump. American Journal of Physiology, 243, R389-R399. 
Bell, R. M., Mocanu, M. M., \& Yellon, D. M. (2011). Retrograde heart perfusion: the Langendorff technique of isolated heart perfusion. Journal of Molecular and Cellular Cardiology, 50, 940-950.

Berne, R. M. (1963). Cardiac nucleotides in hypoxia: possible role in regulation of coronary blood flow. American Journal of Physiology, 204, 317-322.

Bernier, M., Curtis, M. J., \& Hearse, D. J. (1989). Ischemia-induced and reperfusion-induced arrhythmias: Importance of heart rate. American Journal of Physiology, 256, H21-H31.

Cooper, M. W. (1993). Postextrasystolic potentiation. Do we really know what it means and how to use it? Circulation, 88, 2962-2971.

Curtis, M. J. (1998). Characterisation, utilisation and clinical relevance of isolated perfused heart models of ischaemia-induced ventricular fibrillation. Cardiovascular Research, 39, 194-215.

Curtis, M. J., Hancox, J. C., Farkas, A., Wainwright, C. L., Stables, C. L., Saint, D. A., ... Walker, M. J. (2013). The Lambeth Conventions (II): guidelines for the study of animal and human ventricular and supraventricular arrhythmias. Pharmacology and Therapeutics, 139, 213-248.

Duncker, D. J., \& Merkus, D. (2007). Exercise hyperaemia in the heart: the search for the dilator mechanism. The Journal of Physiology, 583, 847-854.

Duncker, D. J., Bache, R. J., \& Merkus, D. (2012). Regulation of coronary resistance vessel tone in response to exercise. Journal of Molecular and Cellular Cardiology, 52, 802-813.

Duncker, D. J., Stubenitsky, R., \& Verdouw, P. D. (1998). Autonomic control of vasomotion in the porcine coronary circulation during treadmill exercise: evidence for feedforward beta-adrenergic control. Circulation Research, 82, 1312-1322.

von Engelhardt, W. (1977). Cardiovascular effects of exercise and training in horses. Advances in Veterinary Science and Comparative Medicine, 21, 173-205.

Farkas, A., \& Curtis, M. J. (2002). Limited antifibrillatory effectiveness of clinically relevant concentrations of class I antiarrhythmics in isolated perfused rat hearts. Journal of Cardiovascular Pharmacology, 39, 412-424.

Farkas, A., \& Curtis, M. J. (2003). Does OT widening in the Langendorff-perfused rat heart represent the effect of repolarization delay or conduction slowing? Journal of Cardiovascular Pharmacology, 42, 612-621.

Farkas, A. S., \& Nattel, S. (2010). Minimizing repolarization-related proarrhythmic risk in drug development and clinical practice. Drugs, 70, 573-603.

Farkas, A. S., Acsai, K., Tóth, A., Dézsi, L., Orosz, S., Forster, T., ... Farkas, A. (2006). Importance of

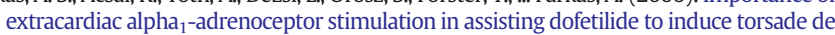
pointes in rabbit hearts. European Journal of Pharmacology, 537, 118-125.

Farkas, A. S., Makra, P., Csík, N., Orosz, S., Shattock, M. J., Fülöp, F., ... Farkas, A. (2009). The role of the $\mathrm{Na}^{+} / \mathrm{Ca}^{2+}$ exchanger, I(Na) and I(CaL) in the genesis of dofetilide-induced torsades de pointes in isolated, AV-blocked rabbit hearts. British Journal of Pharmacology, 156, 920-932.

Farkas, A. S., Rudas, L., Makra, P., Csík, N., Leprán, I., Forster, T., ... Farkas, A. (2010). Biomarkers and endogenous determinants of dofetilide-induced torsades de pointes in alpha(1)-adrenoceptor-stimulated, anaesthetized rabbits. British Journal of Pharmacology, 161, 1477-1495.

Farkas, A., Qureshi, A., \& Curtis, M. J. (1999). Inadequate ischaemia-selectivity limits the antiarrhythmic efficacy of mibefradil during regional ischaemia and reperfusion in the rat isolated perfused heart. British Journal of Pharmacology, 128, 41-50.

Khouri, E. M., Gregg, D. E., \& Rayford, C. R. (1965). Effect of exercise on cardiac output, left coronary flow and myocardial metabolism in the unanesthetized dog. Circulation Research, 17, 427-437.

Kingma, J. G., Jr., \& Rouleau, J. R. (2007). Coronary vasoregulation in health and disease. The Canadian Journal of Cardiology, 23(Suppl B), 9B-14B.

Koizumi, K., Terui, N., \& Kollai, M. (1985). Effect of cardiac vagal and sympathetic nerve activity on heart rate in rhythmic fluctuations. Journal of the Autonomic Nervous System, 12, 251-259.

Langendorf, O. (1895). Untersuchungen am überlebenden säugethierherzen. Pflügers Archiv für die Gesamte Physiologie des Menschen und der Tiere, 61, 291-332.

Laughlin, M. H., Klabunde, R. E., Delp, M. D., \& Armstrong, R. B. (1989). Effects of dipyridamole on muscle blood flow in exercising miniature swine. American Journa of Physiology, 257, H1507-H1515.

Manohar, M. (1988). Left ventricular oxygen extraction during submaximal and maximal exertion in ponies. The Journal of Physiology, 404, 547-556.

Pugsley, M. K., Authier, S., \& Curtis, M. J. (2008). Principles of safety pharmacology. British Journal of Pharmacology, 154, 1382-1399.

Rouleau, J. R., Simard, D., Rodrigue, N., Blouin, A., \& Kingma, J. G., Jr. (2002). Myocardial blood flow after chronic cardiac decentralization in anesthetized dogs: effects of ACE-inhibition. Autonomic Neuroscience, 97, 12-18.

Vatner, S. F., Higgins, C. B., Millard, R. W., \& Franklin, D. (1974). Role of the spleen in the peripheral vascular response to severe exercise in untethered dogs. Cardiovascular Research, 8, 276-282.

Vial, C., Owen, P., Opie, L. H., \& Posel, D. (1987). Significance of release of adenosine triphosphate and adenosine induced by hypoxia or adrenaline in perfused rat heart. Journal of Molecular and Cellular Cardiology, 19, 187-197.

Vincze, D., Farkas, A. S., Rudas, L., Makra, P., Csík, N., Leprán, I., ... Farkas, A. (2008). Relevance of anaesthesia for dofetilide-induced torsades de pointes in alpha ${ }_{1-}$ adrenoceptor-stimulated rabbits. British Journal of Pharmacology, 153, 75-89.

Wiggers, C. J. (1909). The innervation of the coronary vessel. American Journal of Physiology, 24, 391-405.

Yokokawa, M., Kim, H. M., Good, E., Crawford, T., Chugh, A., Pelosi, F., Jr., ... Bogun, F. (2012). Impact of QRS duration of frequent premature ventricular complexes on the development of cardiomyopathy. Heart Rhythm, 9, 1460-1464. 


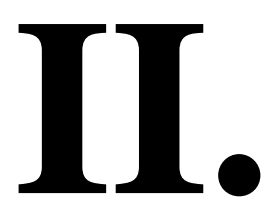




\title{
APPLICATION OF VENTRICULAR TACHYARRHYTHMIA DEFINITIONS OF THE UPDATED LAMBETH CONVENTIONS PROVIDES INCOMPATIBILITY WITH EARLIER RESULTS, MASKS ANTIFIBRILLATORY ACTIVITY AND REDUCES INTER-OBSERVER AGREEMENT
}

\begin{abstract}
${ }^{1}$ Second Department of Medicine and Cardiology Centre, Faculty of Medicine, University of Szeged, Szeged, Hungary; ${ }^{2}$ Department of Medical Physics and Informatics, University of Szeged, Szeged, Hungary; ${ }^{3}$ Bolyai Institute, University of Szeged, Szeged, Hungary; ${ }^{4}$ Department of Pharmacology and Pharmacotherapy, Faculty of Medicine, University of Szeged, Szeged, Hungary; ${ }^{5}$ MTA-SZTE Research Group for Cardiovascular Pharmacology, Hungarian Academy of Sciences, Szeged, Hungary
\end{abstract}

The Lambeth Conventions (LC I), a landmark guidance document for arrhythmia research was updated and arrhythmia definitions were changed in the new Lambeth Conventions II (LC II). This study examined whether the arrhythmia definitions of LC I and LC II yield the same qualitative results and whether LC II improves inter-observer agreement. Two independent investigators performed blinded arrhythmia analysis of the electrocardiograms of isolated, Langendorff rat hearts subjected to regional ischemia and perfused with Class I antiarrhythmics with 3 or $5 \mathrm{mM} \mathrm{K}^{+}$in the perfusate. Data obtained with arrhythmia definitions of LC I and LC II were compared within and between observers. Applying ventricular fibrillation (VF) definition of LC II significantly increased VF incidence and reduced VF onset time irrespective of treatment by detecting 'de novo' VF episodes not found by LC I. LC II reduced the number of ventricular tachycardia (VT) episodes and simultaneously increased the number of VF episodes as compared with the respective values obtained according to LC I. Using VF definition of LC II masked the significant antifibrillatory effects of flecainide and the high $\mathrm{K}^{+}$concentration identified with the VF definition of LC I. When VF incidence was tested, a very strong inter-observer agreement was found according to LC I, whereas using VF definition of LC II reduced interobserver agreement. It is concluded that LC II shifts some tachyarrhythmias from VT to VF class, and thus results obtained by arrhythmia definitions of LC I and LC II are not compatible; VF definition of LC II may change the conclusion of pharmacological, physiological and pathophysiological arrhythmia investigations and may reduce interobserver agreement. Thus, VT and VF definitions of LC II should be amended in order to increase compatibility and inter-observer agreement.

Key words: Lambeth Conventions, arrhythmia definitions, ventricular fibrillation, incompatibility of the results, inter-observer agreement

\section{INTRODUCTION}

Sudden cardiac death caused by ventricular arrhythmias is a major public health problem in modern industrialized countries (1), which emphasizes the importance of arrhythmia research. Currently, there are clinical $(1,2)$ and experimental guidelines $(3,4)$ dealing with arrhythmia research and management, but these guidelines do not use unified arrhythmia definitions, and that is a notable problem when we try to objectively evaluate and compare results of arrhythmia investigations.

The Lambeth Conventions (LC I), a guidance for research on arrhythmias published in 1988 (3), had a substantial impact on the experimental arrhythmia research; the paper has been cited more than 1000 times since its publication 30 years ago according to the database of Web of Science. However, the advances in technology, development of monitoring and pharmacologic solutions and of course the extensive research about arrhythmias had finally led to the realization that LC I was in need to be updated. Thus, a meeting was held in London in 2010 to update the guidance. The revised conventions were intended to be of practical value in terms of the design, execution, and analysis of experiments, with emphasis on the definition, classification, and quantification of ventricular and atrial arrhythmias (4). The revised Lambeth Conventions (LC II) were intended to be applied in preclinical and clinical research. Authors of LC II invited investigators to state whether or not they had used the conventions in their studies, and to test their validity by experiment.

Importantly, there are substantial changes in the definitions of the ventricular tachyarrhythmias between the original and the updated Lambeth Conventions (Table 1). The new 
tachyarrhythmia definitions imply that some arrhythmias classified as ventricular tachycardia (VT) according to LC I are now classified as ventricular fibrillation (VF) according to LC II. This suggests that the new definitions change the results and more importantly - the conclusion of arrhythmia studies. Thus, the aim of the present study was to examine whether the arrhythmia definitions of LC I and LC II are compatible, and yield the same qualitative arrhythmia results. Also, it was tested whether arrhythmia definitions of LC I or LC II allow better inter-observer agreement. Thus, two independent investigators reanalysed the electrocardiogram (ECG) recordings of experiments done earlier by Farkas and Curtis (5), and performed a retrospective, blinded analysis of the number and incidence of ventricular arrhythmias in isolated, Langendorff perfused rat hearts subjected to regional ischemia and treated with Class I antiarrhythmic agents. The arrhythmia data obtained by applying arrhythmia definitions of LC I and LC II were compared to test the compatibility between the arrhythmia definitions of LC I and LC II. Also, inter-observer agreement was determined to investigate whether arrhythmia definitions of LC I or LC II allow

Table 1. Comparison of the definitions of ventricular arrhythmias between the Lambeth Conventions I and II.

\begin{tabular}{|c|c|c|}
\hline Arrhythmia & $\begin{array}{c}\text { Definition according to } \\
\text { Lambeth Conventions I (3) }\end{array}$ & $\begin{array}{c}\text { Definition according to } \\
\text { Lambeth Conventions II (4) }\end{array}$ \\
\hline $\begin{array}{l}\text { Ventricular premature beat } \\
\text { (VPB) }\end{array}$ & $\begin{array}{l}\text { Isolated ventricular premature } \\
\text { beats are defined as discrete } \\
\text { and identifiable premature } \\
\text { QRS complexes (premature } \\
\text { in relation to the P wave). }\end{array}$ & $\begin{array}{l}\text { A VPB is defined as a } \\
\text { ventricular electrical complex } \\
\text { (complete electrical event: } \\
\text { QRS, RS, QRST or RST) that } \\
\text { is different in shape (voltage } \\
\text { and/or duration, i.e., height } \\
\text { and/or width) from the } \\
\text { preceding (non-VPB) } \\
\text { ventricular complex, and is } \\
\text { premature in relation to the } \\
\text { preceding ventricular } \\
\text { complex. }\end{array}$ \\
\hline Bigeminy & $\begin{array}{l}\text { Bigeminy is characterised by } \\
\text { the minimum sequence: P, } \\
\text { QRS, VPB, P, QRS, VPB. }\end{array}$ & $\begin{array}{l}\text { Bigeminy has the minimum } \\
\text { sequence VPB, normal sinus } \\
\text { beat, VPB (which may be } \\
\text { repeated) in which the VPBs } \\
\text { have the same shape and } \\
\text { timing. }\end{array}$ \\
\hline Salvo & $\begin{array}{l}\text { Two or three consecutive } \\
\text { VPBs do not constitute } \\
\text { ventricular tachycardia but } \\
\text { should be termed a salvo. }\end{array}$ & $\begin{array}{l}\text { A run of two or three } \\
\text { consecutive VPBs is defined } \\
\text { as a salvo. }\end{array}$ \\
\hline $\begin{array}{l}\text { Ventricular tachycardia } \\
\text { (VT) }\end{array}$ & $\begin{array}{l}\text { A run of } 4 \text { or more } \\
\text { consecutive ventricular } \\
\text { premature beats. }\end{array}$ & $\begin{array}{l}\text { A sequence of a minimum of } \\
4 \text { consecutive ventricular } \\
\text { complexes. } \\
\text { Monomorphic VT: } \\
\text { peak-peak interval, height } \\
\text { and intrinsic shape are } \\
\text { constant. } \\
\text { Polymorphic VT: } \\
\text { the peak-peak interval and/or } \\
\text { height and/or intrinsic shape } \\
\text { vary, and the variation of any } \\
\text { or each of these is } \\
\text { progressive. }\end{array}$ \\
\hline $\begin{array}{l}\text { Ventricular fibrillation } \\
\text { (VF) }\end{array}$ & $\begin{array}{l}\text { A signal for which individual } \\
\text { QRS deflections can no } \\
\text { longer be distinguished from } \\
\text { one another (implying } \\
\text { morphological instability) } \\
\text { and for which a rate can no } \\
\text { longer be measured. }\end{array}$ & $\begin{array}{l}\text { A sequence of a minimum of } \\
4 \text { consecutive ventricular } \\
\text { complexes without } \\
\text { intervening diastolic pauses, } \\
\text { in which intrinsic shape, } \\
\text { peak-peak interval and height } \\
\text { vary, and the variation } \\
\text { between each is non- } \\
\text { progressive. It is the non- } \\
\text { progressive nature of the } \\
\text { variation of all } 3 \text { variables } \\
\text { that distinguishes VF from } \\
\text { polymorphic VT and torsades } \\
\text { de pointes. }\end{array}$ \\
\hline
\end{tabular}


better agreement on the arrhythmia results between the two independent observers.

\section{MATERIALS AND METHODS}

General experimental procedure

For detailed methodical description see the published study by Farkas and Curtis (5), from which the raw ECGs were obtained for arrhythmia analysis. Thus, here only a short description of the experiments is added as follows.

The animal-handling protocol was in accordance with the Guidance of the Operation on the Animals (Scientific Procedures) Act 1986, London, UK. Male rats were anesthetized with pentobarbitone $(60 \mathrm{mg} / \mathrm{kg}$ intraperitoneally). Hearts were excised, and then perfused according to Langendorff with modified Krebs solution containing $118.5 \mathrm{mM} \mathrm{NaCl}, 25.0 \mathrm{mM}$ $\mathrm{NaHCO}_{3}, 1.2 \mathrm{mM} \mathrm{MgSO}_{4}, 1.2 \mathrm{mM} \mathrm{NaH}_{2} \mathrm{PO}_{4}, 1.4 \mathrm{mM} \mathrm{CaCl}_{2}, 3$ $\mathrm{mM} \mathrm{KCl}$ (or $5 \mathrm{mM}$ where indicated), and $11.1 \mathrm{mM}$ glucose. Perfusion solution was delivered at $37^{\circ} \mathrm{C}$ and $\mathrm{pH}$ 7.4; perfusion pressure was maintained constant at $70 \mathrm{~mm} \mathrm{Hg}$. The left main coronary artery was occluded by a silk suture for $30 \mathrm{~min}$. A unipolar ECG was recorded using a MacLab system (ADInstruments Ltd., Oxford, UK) (5-7).

Drug administration protocol, groups of hearts

In the first two sets of experiments, Krebs' solution contained $3 \mathrm{mM} \mathrm{K}^{+}$. In the third and fourth sets of experiments, $\mathrm{K}^{+}$ concentration was elevated to $5 \mathrm{mM}$. Each set of experiments contained four groups of hearts: one control and three drug-treated groups, each treated with one concentration of a representative Class I antiarrhythmic (quinidine, lidocaine or flecainide). One lower and one higher concentration of quinidine $(0.79$ and 7.90 $\mu \mathrm{M})$, lidocaine (3.88 and $12.93 \mu \mathrm{M})$, and flecainide ( 0.74 and 1.48 $\mu \mathrm{M})$, representing the peak unbound plasma and total blood concentrations, respectively, at 'therapeutic' dosage, were evaluated in the first two sets of experiments and also in the last two sets of experiments. Each group contained 12 hearts in the first two sets of experiments ( 96 hearts in total; $3 \mathrm{mM} \mathrm{K}^{+}$in the Krebs), whereas every group in the third and fourth sets of experiments contained 6 hearts (48 hearts in total, $5 \mathrm{mM} \mathrm{K}^{+}$in the Krebs). Experiments were done in a randomized and blinded manner.

Reanalysing ischaemic arrhythmias according to definitions of LC I and LC II, measurement of the number and incidence of tachyarrhythmias

The ECG recordings of the original investigation done by Farkas and Curtis (5) were reanalysed by two independent investigators using LabChart7 (ADInstruments Ltd., Oxford, UK). The two investigators were well trained and equally experienced in evaluating ECG. Ventricular arrhythmias were defined according to the LC I for the primary evaluation (Table 1). The number of VT and VF episodes, the incidence and the time to onset of VT and VF were determined from the ECG recorded during the 30-min-long ischaemia. Then the whole analysis was repeated according to the arrhythmia definitions of LC II (Table 1). All variables were measured in a blinded manner.

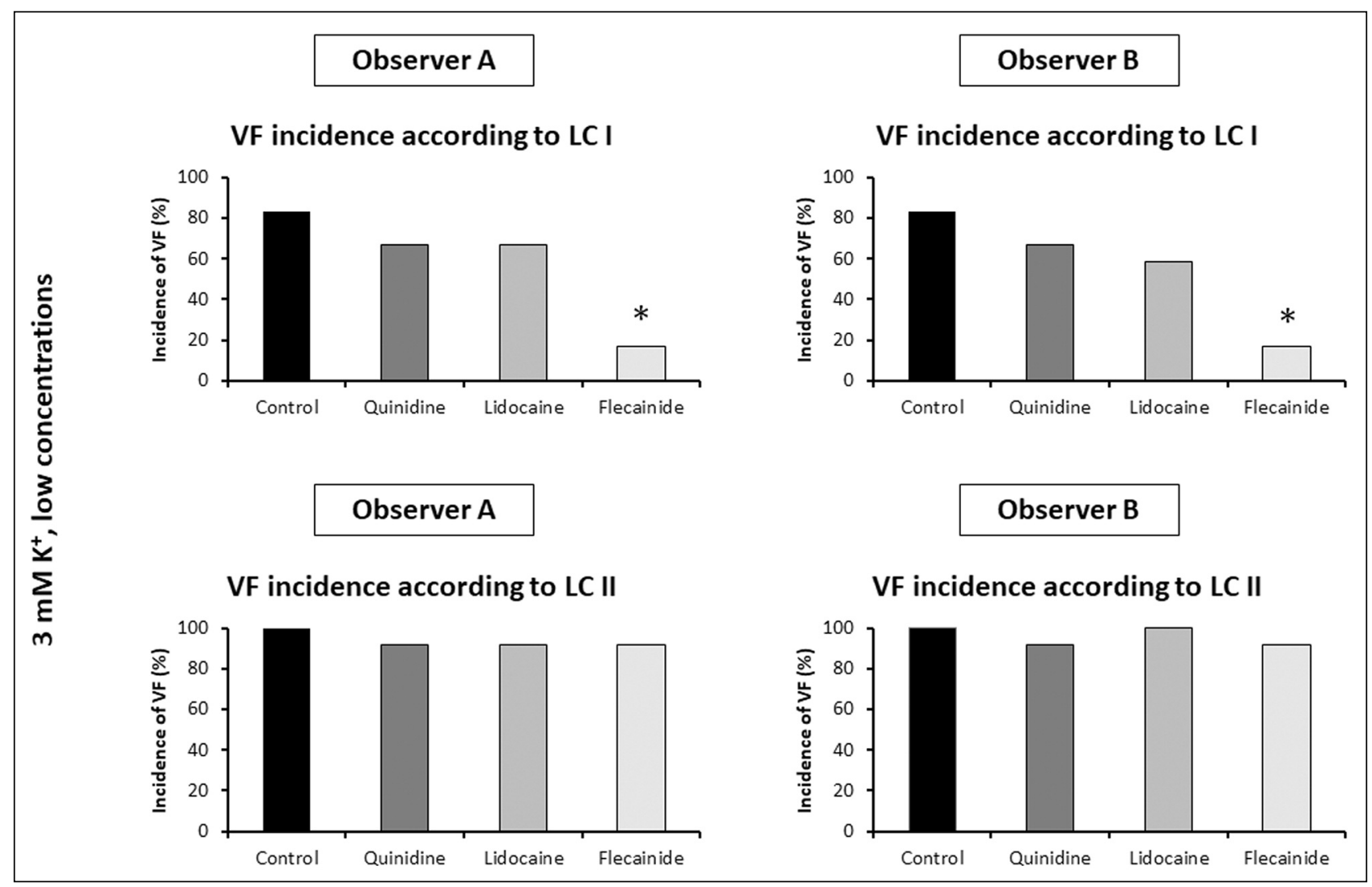

Fig. 1. Comparison of the percent incidences of ischaemic ventricular fibrillation (VF) obtained from the $1^{\text {st }}$ set of experiments by two independent observers (Observer A and Observer B) using VF definition of Lambeth Conventions I (LC I) and Lambeth Conventions II (LC II). Langendorff perfused rat hearts were subjected to local ischaemia for 30 min. Hearts were perfused with the low

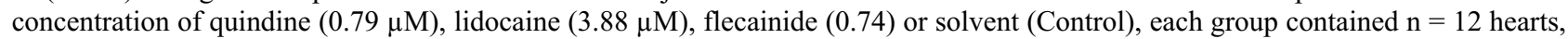
Krebs solution contained $3 \mathrm{mM} \mathrm{K}^{+} . * \mathrm{P}<0.05$ versus Control. 


\section{Statistical analysis}

Continuous variables were expressed as means \pm SEM. Within group comparison of continuous data (e.g. the number of arrhythmia episodes, the onset times of VT and VF) were performed with the non-parametric Wilcoxon test for paired samples. Arrhythmia incidences were expressed as percent values, and were compared with Fisher's exact test with the Bonferroni correction, that is, the $\mathrm{P}$ values of Fisher's exact test were multiplied by the numbers of comparisons to allow multiple comparisons (8). A P value $<0.05$ was taken as indicative of a statistically significant difference between values. The intraobserver agreement on arrhythmia incidence data obtained according to LC I and LC II was assessed with Cohen's kappa statistic (9). Similarly, the inter-observer agreement on VT and VF incidence data among independent observers was calculated with Cohen's kappa statistic (9).

\section{RESULTS}

Ischaemic ventricular fibrillation incidence in the first set of experiments ( $3 \mathrm{mM} \mathrm{K} \mathrm{K}^{+}$, low concentrations of drugs)

When the arrhythmia definitions of LC I were applied, both of the two independent investigators found that VF was frequently induced in the control group, and only flecainide was able to reduce VF incidence significantly at the applied low concentration (Fig. 1). These results are in a good accordance with the arrhythmia results of the original investigation obtained by a different observer using the same arrhythmia definitions of LC I (5). This means that applying arrhythmia definitions of LC I allowed the two independent investigators to reproduce previous results.

When arrhythmia definitions of LC II were applied, both of the two independent investigators found that VF was induced in all control hearts, and none of the drugs affected significantly VF incidence at the applied low concentrations (Fig. 1). Thus, the significant antifibrillatory effect of the low concentration of flecainide was masked when arrhythmia definitions of LC II were used. This means that results about the antifibrillatory effect of flecainide qualitatively differ from those obtained by applying arrhythmia definitions of LC I.

Ischaemic ventricular fibrillation incidence in the second set of experiments ( $3 \mathrm{mM} \mathrm{K} \mathrm{K}^{+}$, high concentrations of drugs)

When the arrhythmia definitions of LC I were applied, both of the two independent investigators found that VF was frequently induced in the control group, and all three drugs reduced VF incidence significantly at the applied high concentrations (Fig. 2). These results well accord with the arrhythmia results of the original investigation obtained by a different observer using the same arrhythmia definitions of LC I (5). This means that applying arrhythmia definitions of LC I allowed the two independent investigators to reproduce previous results.

When the arrhythmia definitions of LC II were applied, Observer B found that the high concentrations of all three drugs

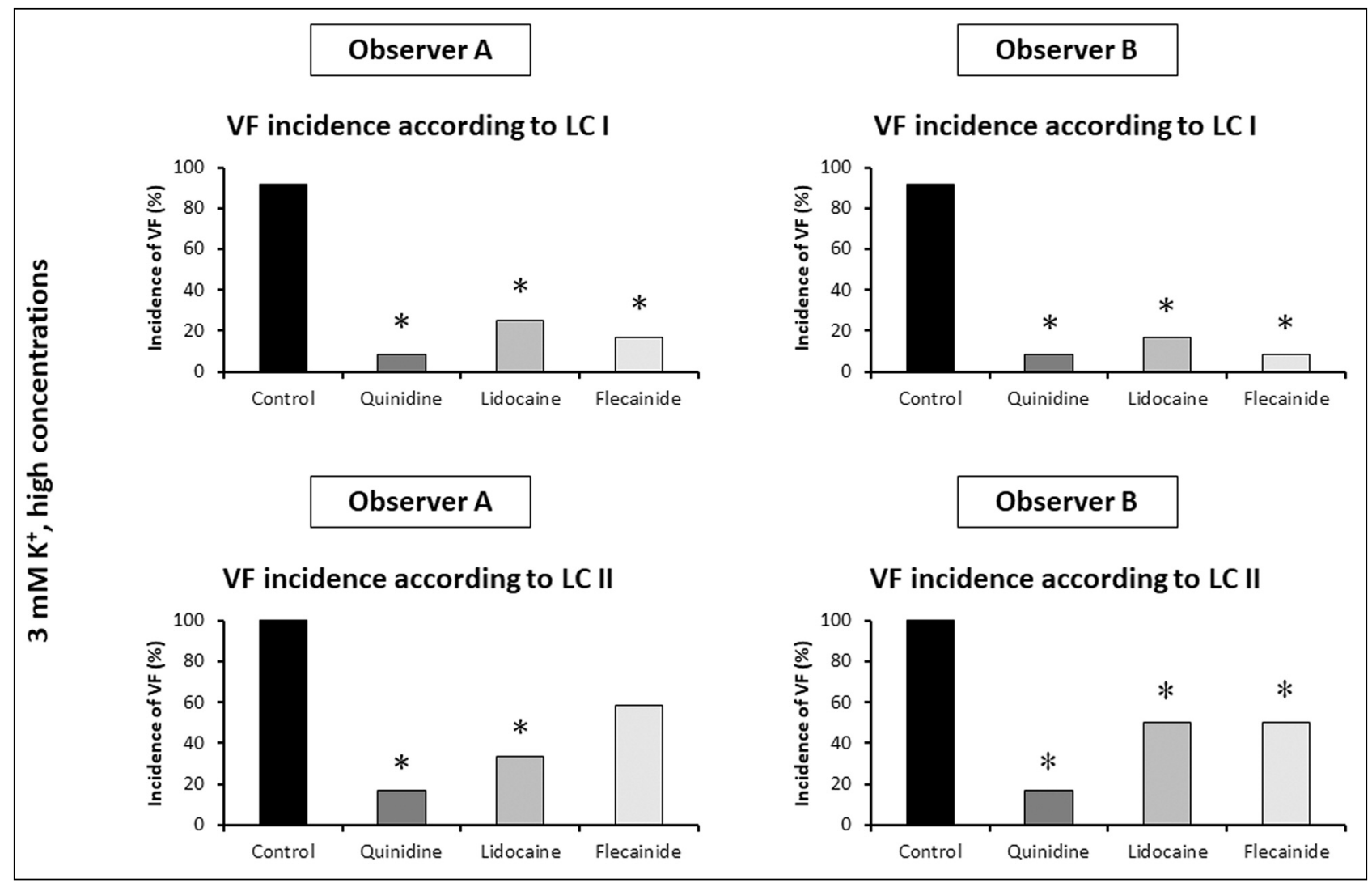

Fig. 2. Comparison of the percent incidences of ischaemic ventricular fibrillation (VF) obtained from the $2^{\text {nd }}$ set of experiments by two independent observers (Observer A and Observer B) using VF definition of Lambeth Conventions I (LC I) and Lambeth Conventions II (LC II). Langendorff perfused rat hearts were subjected to local ischaemia for $30 \mathrm{~min}$. Hearts were perfused with the high concentration of quindine $(7.90 \mu \mathrm{M})$, lidocaine $(12.93 \mu \mathrm{M})$, flecainide $(1.48 \mu \mathrm{M})$ or solvent $(\mathrm{Control})$, each group contained $\mathrm{n}=12$ hearts, Krebs solution contained $3 \mathrm{mM} \mathrm{K}^{+} . * \mathrm{P}<0.05$ versus Control. 
reduced the incidence of ischaemic VF as compared with control, which accords with the results obtained according to LC I (Fig. 2). However, Observer A found that the applied high concentration of flecainide did not significantly reduce the incidence of ischaemic VF, which qualitatively differs from the results obtained according to LC I, and it also contradicts the results of Observer B (Fig. 2).
Ischaemic ventricular fibrillation incidence in the control hearts, the effect of $\mathrm{K}^{+}$concentration on ventricular fibrillation incidence

Ischaemic VF incidence was compared between the control group of hearts perfused with $3 \mathrm{mM} \mathrm{K}^{+}$(the hearts of the control groups of the $1^{\text {st }}$ and $2^{\text {nd }}$ sets of experiments) and the control hearts perfused with $5 \mathrm{mM} \mathrm{K}^{+}$(the hearts of the control groups

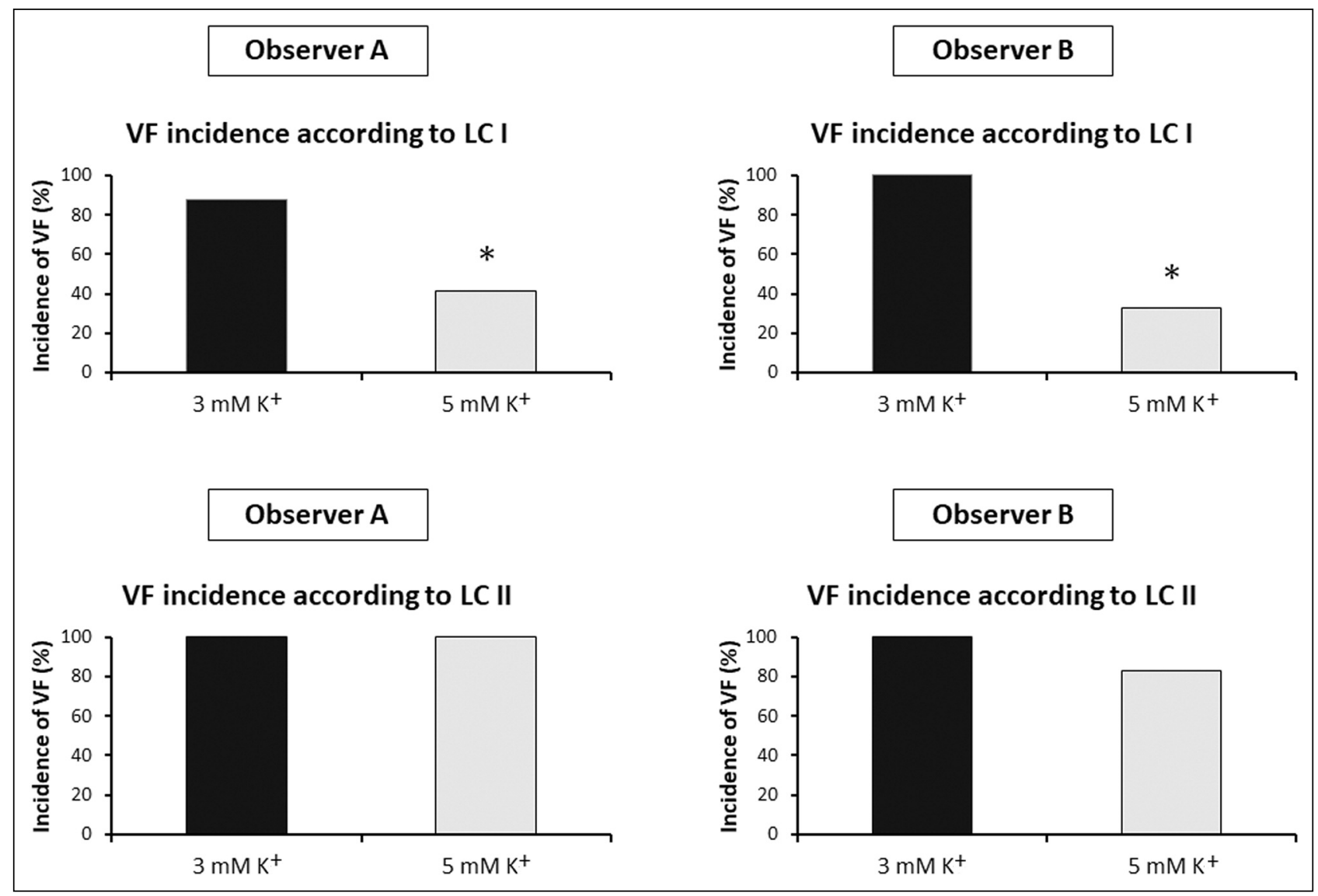

Fig. 3. Comparison of the percent incidences of ischaemic ventricular fibrillation (VF) between control rat hearts subjected to local ischaemia for $30 \mathrm{~min}$ and perfused with Krebs solution containing 3 or $5 \mathrm{mM} \mathrm{K}^{+}(\mathrm{n}=24$ and $\mathrm{n}=12$ hearts, respectively). Two independent observers (Observer A and Observer B) performed the blinded arrhythmia analysis using VF definition of Lambeth Conventions I (LC I) and Lambeth Conventions II (LC II). ${ }^{*} \mathrm{P}<0.05$ versus $3 \mathrm{mM} \mathrm{K}{ }^{+}$.

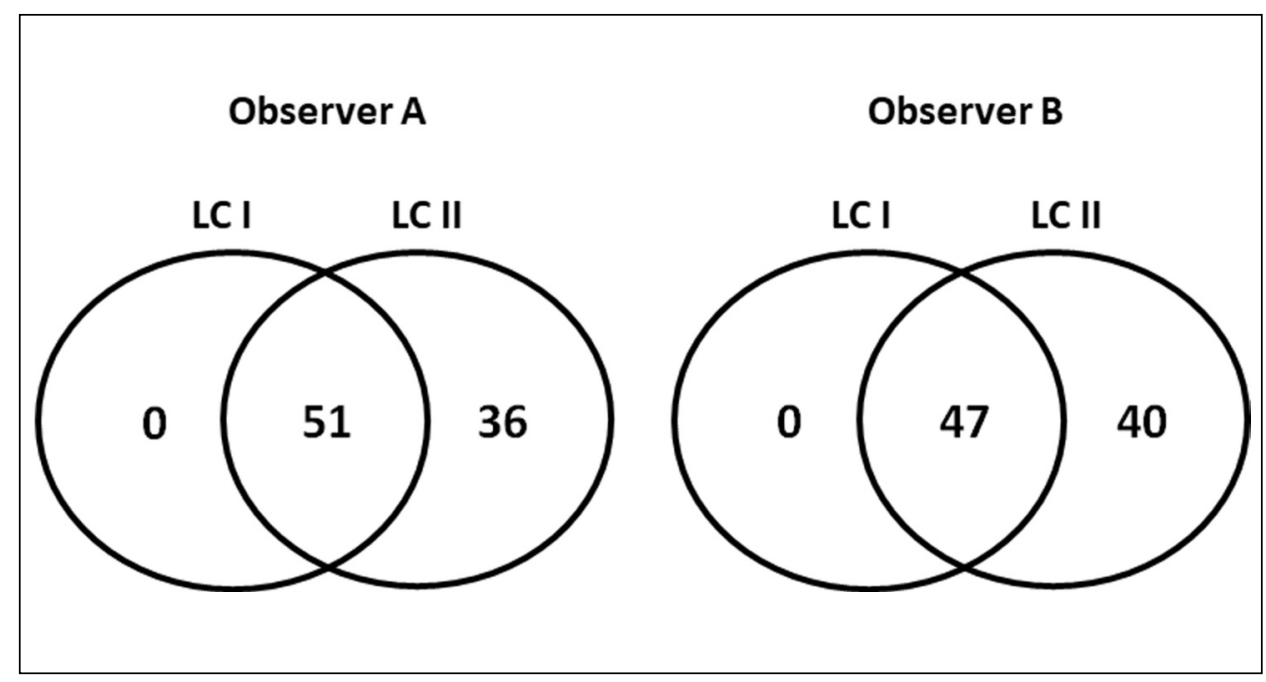

Fig. 4. The total number of hearts with ischaemic ventricular fibrillation (VF) out of the 144 Langendorff perfused rat hearts subjected to local ischaemia for 30 min irrespective of the drug treatment and the $\mathrm{K}^{+}$content of the perfusate. VF was diagnosed by applying VF definitions of Lambeth Conventions I (LC I) and Lambeth Conventions II (LC II). Observer A and Observer B are the two independent observers. The number in the common part of the LC I and LC II classes shows the total number of hearts that experienced $\mathrm{VF}$ according to both LC I and LC II. 
of the $3^{\text {rd }}$ and $4^{\text {th }}$ sets of experiments). Both investigators found that the elevation of the $\mathrm{K}^{+}$concentration from $3.0 \mathrm{mM}$ to 5.0 $\mathrm{mM}$ significantly reduced the incidence of ischaemic $\mathrm{VF}$, when arrhythmia definitions of LC I were applied (Fig. 3). These results well accord with the arrhythmia results of the original investigation obtained by a different observer using the same arrhythmia definitions of LC I (5). Again, this shows that applying arrhythmia definitions of LC I allowed the two independent investigators to reproduce previous results. However, the marked antiarrhythmic effect of the increased $\mathrm{K}^{+}$ concentration seen according to LC I was masked when arrhythmia definitions of LC II were applied in the present investigation, and these results did not differ qualitatively between the two investigators (Fig. 3).

Table 2. The percent incidences of ischaemic ventricular tachycardia and ventricular fibrillation irrespective of the drug treatment and $\mathrm{K}^{+}$ content of the perfusate in $n=144$ Langendorff perfused rat hearts subjected to local ischaemia for 30 min. Intra-observer agreement on the results obtained according to the arrhythmia definitions of Lambeth Conventions I and Lambeth Conventions II, results of the Cohen's Kappa statistical analysis. Observers A and B are the two independent observers of the present investigation. LC I and LC II, VT and VF incidence data obtained according to the arrhythmia definitions of Lambeth Conventions I and Lambeth Conventions II, respectively.

\begin{tabular}{|cc|cc|c|c|}
\hline Arrhythmia & Observer & \multicolumn{2}{|c|}{$\begin{array}{c}\text { Incidence } \\
(\%)\end{array}$} & Kappa & $\begin{array}{c}95 \% \text { Confidence } \\
\text { interval } \\
\text { for kappa }\end{array}$ \\
\hline LC I & LC II & & $0.922-1.000$ \\
& A & 85 & 84 & 0.974 & $0.421-0.751$ \\
\hline VF & B & 88 & $76^{*}$ & 0.586 & $0.411-0.646$ \\
& B & 35 & $60^{*}$ & 0.529 & $0.365-0.599$ \\
\hline
\end{tabular}

VT, ventricular tachycardia; VF, ventricular fibrillation; Kappa, kappa coefficient obtained by Cohen's Kappa statistical analysis; note that kappa value of 1.0 means perfect agreement, whereas the kappa value of 0.0 means absolutely no agreement; thus, the greater the kappa value in the range of $0.0-1.0$, the greater the agreement. $* \mathrm{P}<0.05$ versus LC I.

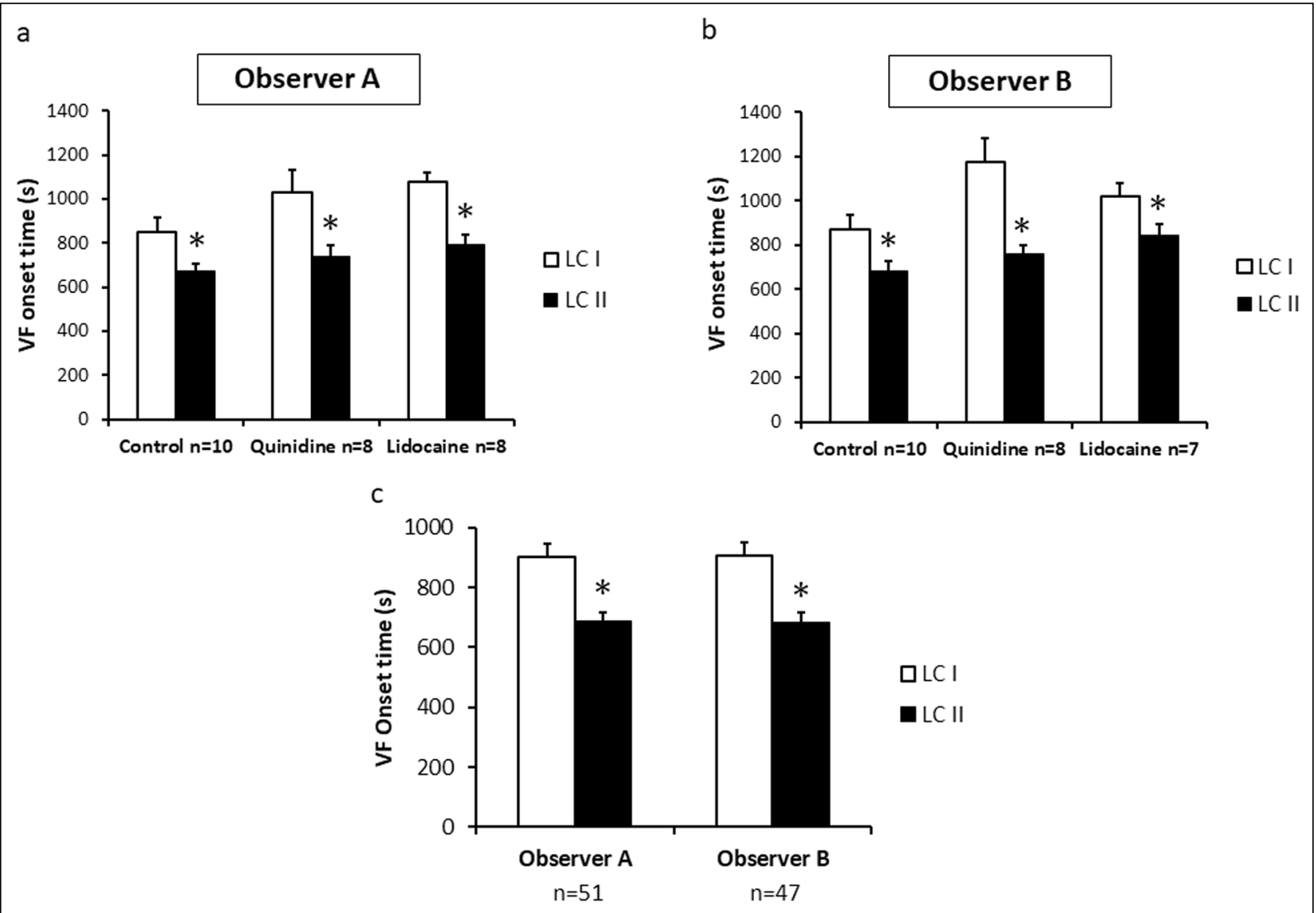

Fig. 5. The effect of definitions of ventricular fibrillation (VF) of Lambeth Conventions I (LC I) and Lambeth Conventions II (LC II) on the onset time of the first episode of ischaemic VF in Langendorff perfused rat hearts subjected to local ischaemia for 30 min and experiencing VF according to both LC I and LC II. Parts (a) and (b): VF onset times in the $1^{\text {st }}$ set of experiments, in which hearts were perfused with the low concentration of quindine $(0.79 \mu \mathrm{M})$, lidocaine $(3.88 \mu \mathrm{M})$, or solvent (Control), Krebs solution contained $3 \mathrm{mM}$ $\mathrm{K}^{+}$. Part (c): VF onset times in the whole set of experiments irrespective of drug treatment and the $\mathrm{K}^{+}$content of the perfusate. Observer $\mathrm{A}$ and Observer $\mathrm{B}$ are the two independent observers. $* \mathrm{P}<0.05$ versus LC I. 
Table 3. Inter-observer agreement on the incidences of ventricular tachycardia and ventricular fibrillation. Results of the Cohen's Kappa statistical analysis. LC I and LC II: VF and VT incidence data obtained according to the arrhythmia definitions of Lambeth Conventions I and Lambeth Conventions II, respectively. Observers A and B are the two independent observers of the present investigation, Observer $\mathrm{O}$ is the observer of the original investigation published by Farkas and Curtis (5).

\begin{tabular}{|c|c|c|c|c|}
\hline $\begin{array}{c}\text { Incidence } \\
\text { of }\end{array}$ & Lambeth Convention & Observers & Kappa & $\begin{array}{c}95 \% \text { Confidence } \\
\text { interval } \\
\text { for kappa }\end{array}$ \\
\hline \multirow{2}{*}{ VT } & LC I & A vs. O & 0.918 & $0.826-1.000$ \\
\cline { 3 - 5 } & & B vs. O & 0.615 & $0.424-0.805$ \\
\cline { 3 - 5 } & A vs. B & 0.652 & $0.471-0.833$ \\
\cline { 2 - 5 } & LC II & A vs. B & 0.675 & $0.525-0.825$ \\
\hline VF & LC I & A vs. O & 0.908 & $0.836-0.980$ \\
\cline { 3 - 5 } & & B vs. O & 0.938 & $0.877-0.998$ \\
\cline { 2 - 5 } & & A vs. B & 0.938 & $0.879-0.998$ \\
\cline { 2 - 5 } & LC II & A vs. B & 0.884 & $0.806-0.962$ \\
\hline
\end{tabular}

VT, ventricular tachycardia; VF, ventricular fibrillation; Kappa, kappa coefficient obtained by Cohen's Kappa statistical analysis. VT and $\mathrm{VF}$ incidences were obtained irrespective of the drug treatment and $\mathrm{K}^{+}$content of the perfusate from $\mathrm{n}=144$ Langendorff perfused rat hearts subjected to local ischaemia for $30 \mathrm{~min}$.

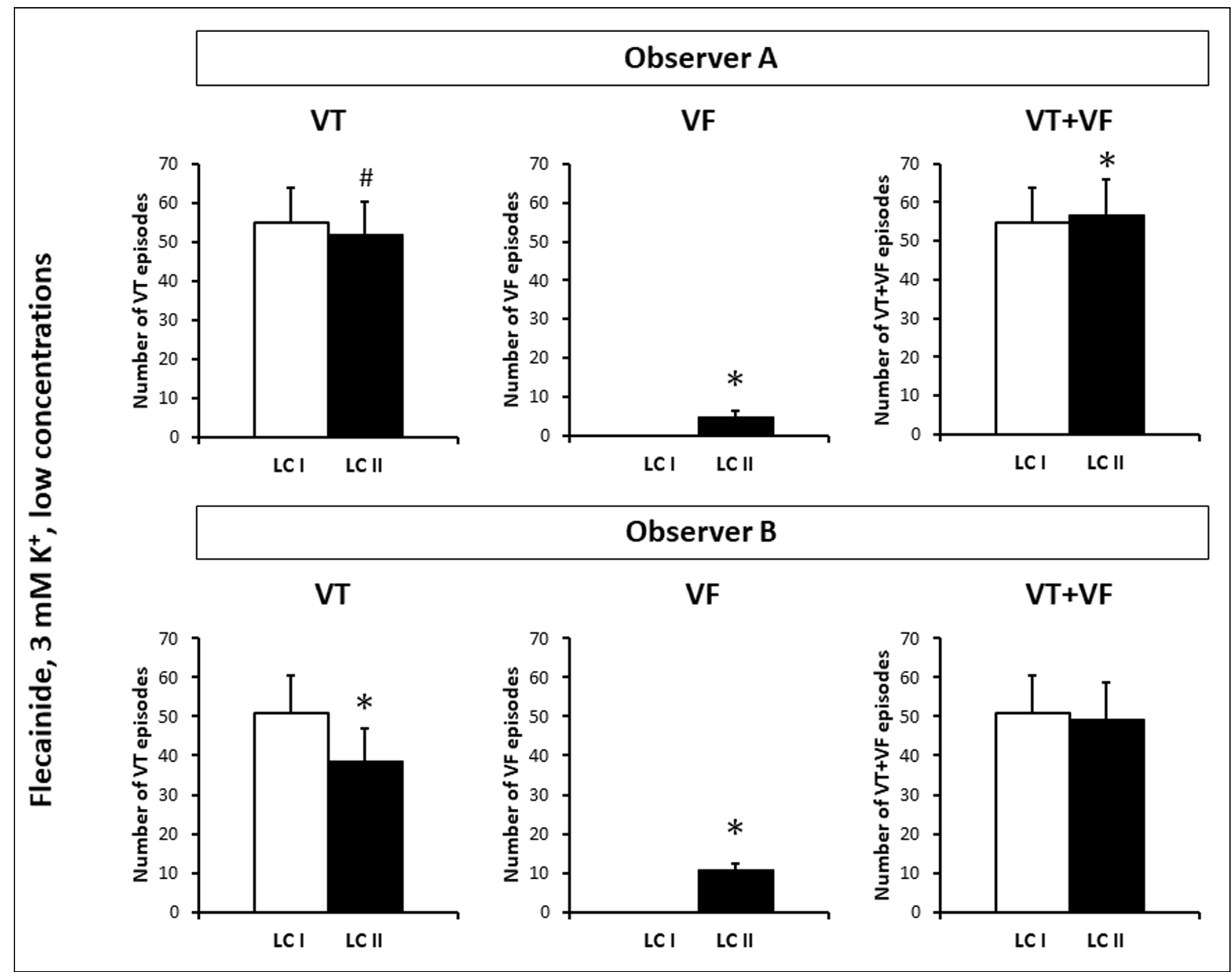

Fig. 6. The numbers of ventricular tachycardia (VT) and ventricular fibrillation (VF) episodes and the cumulative number of VT and VF episodes (VT + VF) in those eight hearts of the flecainide treated group of the first set of experiments, in which neither Observer A nor Observer B found VF according to Lambeth Conventions I (LC I), but both of them identified VF according to Lambeth Conventions II (LC II). Observer A and Observer B are the two independent observers. ${ }^{*} \mathrm{P}<0.05$ versus LC I. \#P $=0.055$ versus LC I. Note that VF episodes found by applying VF definition of LC II are 'de novo' VF episodes, and occurred only as a result of 'arrhythmia shift', and 'arrhythmia fragmentation' did not contribute to their occurrence. 
Ischaemic ventricular tachycardia and ventricular fibrillation incidences in the whole investigation, and intra-observer agreement

VT and VF incidences were calculated irrespective of the treatment and the $\mathrm{K}^{+}$content of the perfusate in the 144 hearts of the whole investigation. Observer A did not find significant effect of LC II on VT incidence, and achieved good intraobserver agreement on VT incidence (Table 2). However, Observer B found that applying arrhythmia definitions of LC II significantly reduced VT incidence as compared with the respective value obtained according to $\mathrm{LC} \mathrm{I}$, which resulted in

Table 4. The effect of applying the new definition of ventricular fibrillation of Lambeth Conventions II on the onset time of ischaemic ventricular fibrillation in Langendorff perfused rat hearts. The onset time of ischaemic VF was examined in all hearts that experienced VF irrespective of $\mathrm{K}^{+}$content of the perfusate and drug treatment in the whole investigation (in all four sets of experiments utilising $\mathrm{n}=144$ isolated rat hearts).

\begin{tabular}{|l|c|c|c|c|}
\hline & \multicolumn{2}{|c|}{ Observer A } & \multicolumn{2}{c|}{ Observer B } \\
\hline & Number of hearts & $\%$ & Number of hearts & $\%$ \\
\hline & & & & \\
\hline VF found in the same heart by both LC I and LC II & 51 & 100 & 47 & 100 \\
\hline VF onset time reduced by LC II & 42 & 82 & 38 & 81 \\
\hline VF onset time not affected by LC II & 9 & 18 & 9 & 19 \\
\hline VF onset time increased by LC II & 0 & 0 & 0 & 0 \\
\hline
\end{tabular}

LC I and LC II, Lambeth Conventions I and II, respectively. VF, ischaemic ventricular fibrillation. Observer A and Observer B, the two independent observers who performed blinded arrhythmia analysis of the experiments.

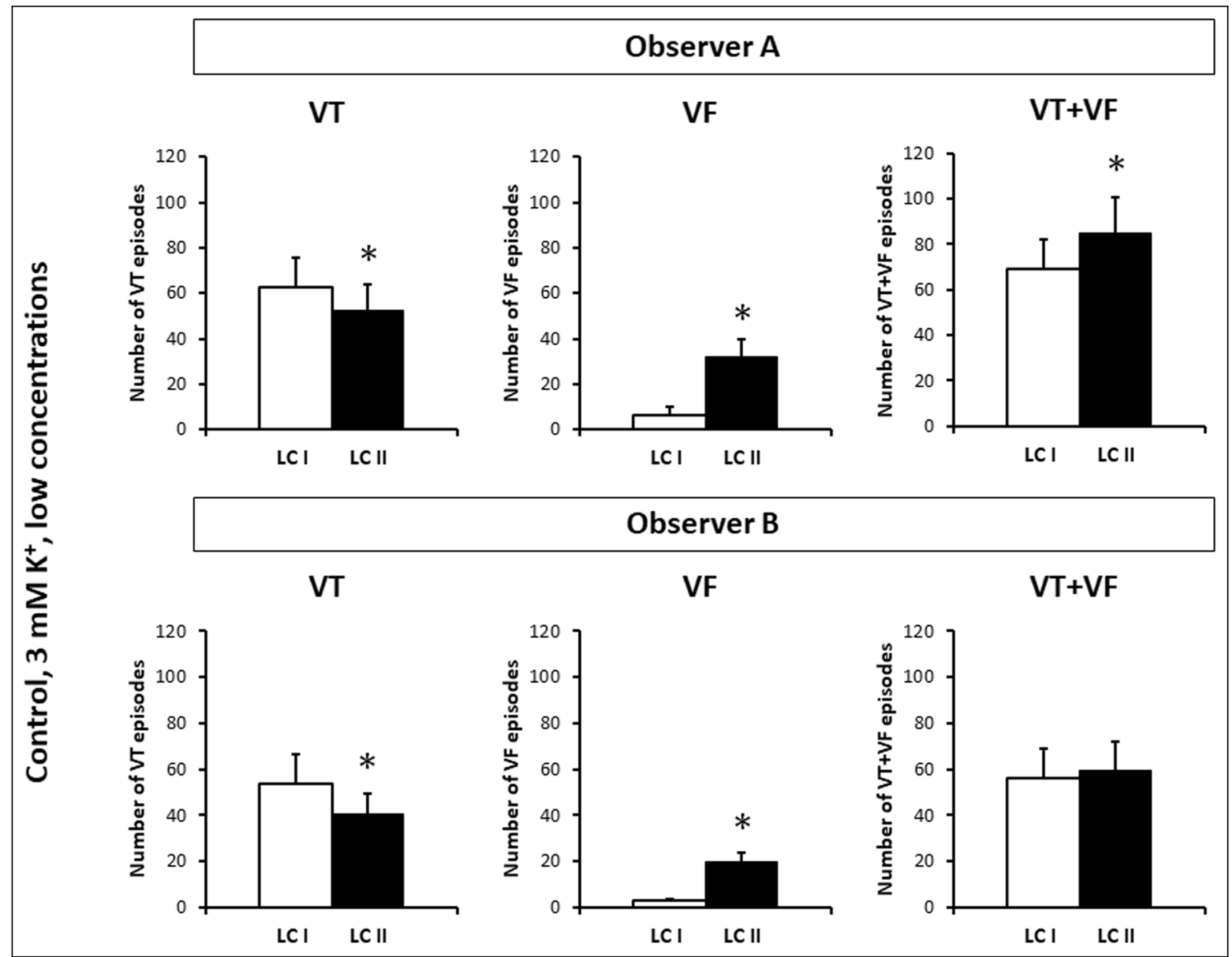

Fig. 7. The numbers of ventricular tachycardia (VT) and ventricular fibrillation (VF) episodes and the cumulative number of VT and VF episodes (VT $+V F$ ) in the $n=12$ control hearts of the first set of the experiments. LC I and LC II: data obtained by using arrhythmia definitions of Lambeth Conventions I and Lambeth Conventions II, respectively. Observer A and Observer B are the two independent observers. ${ }^{*} \mathrm{P}<0.05$ versus LC I. Note, that the extra VF episodes found by applying VF definition of LC II can be the result of 'arrhythmia shift'. However, in theory, 'arrhythmia fragmentation' could also contribute to the increase in VF number according to LC II, as VF episodes found by LC I could be fragmented into shorter episodes due to containing diastolic pauses. 
only moderate intra-observer agreement on VT incidence (Table 2).

Both observers found that VF was diagnosed in a significantly greater proportion of hearts, when the arrhythmia definitions of LC II were applied, as compared with the respective value measured according to the arrhythmia definitions of LC I (Table 2). Also, both observers found that when arrhythmia definitions of LC II were applied, VF was diagnosed in all hearts, in which VF was diagnosed according to LC I. However, there were many hearts, in which VF was diagnosed only according to LC II and VF was not found according to LC I (Fig. 4). Consequently, Cohen's kappa analysis showed that intra-observer agreement on
VF incidence data obtained according to LC I and LC II was only moderate in case of both A and B Observers (Table 2).

The inter-observer agreement on ventricular tachycardia and ventricular fibrillation incidences among independent observers

The inter-observer agreement on VT and VF incidences among independent observers was calculated irrespective of the treatment and the $\mathrm{K}^{+}$concentration in the 144 hearts of the whole investigation. Applying VT definition of LC II did not remarkably improve the inter-observer agreement on VT incidence between Observers A and B; moderate inter-observer

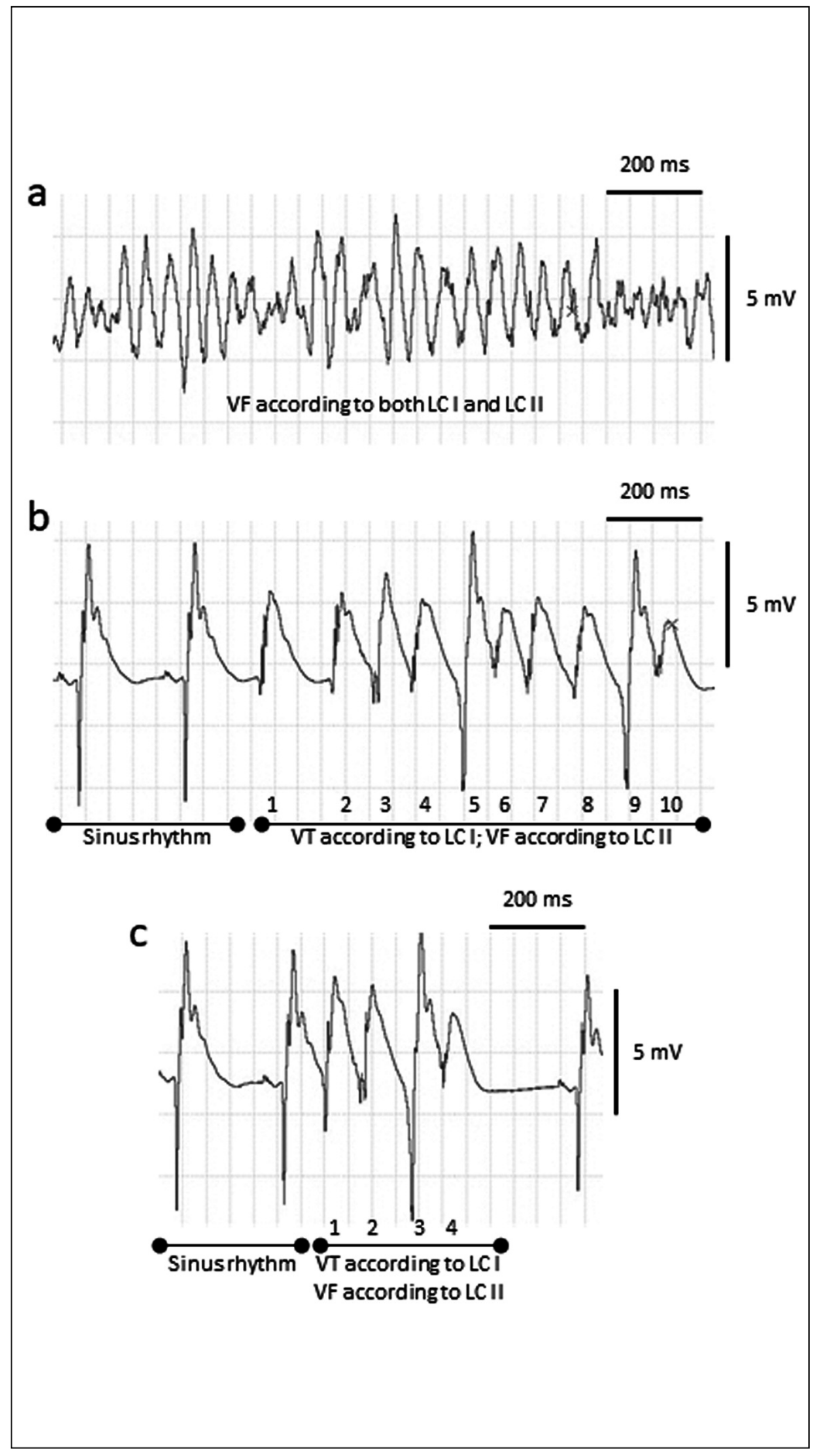

Fig. 8. Incompatibility between the classes of the ventricular fibrillation of the Lambeth Conventions I (LC I) and Lambeth Conventions II (LC II). Part (a): ventricular tachyarrhythmia diagnosed as ventricular fibrillation (VF) according to both LC I and LC II. Part (b): a ten-beat tachyarrhythmia episode of 'de novo' VF as this ventricular tachyarrhythmia is diagnosed as ventricular tachycardia (VT) according to LC I, while the same episode is diagnosed as VF according to LC II. LC II emphasised the importance of 'diastolic pause'. According to LC II, "if an arrhythmia is interrupted by an identifiable pause or quiescence (a 'diastolic pause', or a flat isoelectrical line) that is longer than the prevailing sinus rate (or idioventricular rate if the AV node is blocked) then it makes more sense to classify each arrhythmia present before and after the pause as separate events" (4). Based on the above recommendation of LC II, there is no relevant diastolic pause between the ventricular complexes of this tachyarrhythmia, thus this arrhythmia should be regarded as one single run of arrhythmia. Also note that the arrhythmia consists of sections with four consecutive beats (beats 3-45-6 and beats 7-8-9-10), in which neither intrinsic shape, nor peek-peek interval, nor height of the ventricular complexes change progressively, thus each of these four-beat arrhythmia sections should be classified as VF according to LC II (see Table 1 for the arrhythmia definitions). Convention 14 of LC II states that "When rhythms segue from one to another without an identifiable interruption or quiescence, or vary between simultaneously recorded leads, then the arrhythmia should be classified as the most serious manifestation within segue or lead" (4). As this arrhythmia in part b should be regarded as one single run of arrhythmia and VF is the most serious arrhythmia manifestation within segue, the whole arrhythmia should be classified as VF according to LC II. On the other hand, QRS deflections can be distinguished from one another and the rate can be measured, thus the same arrhythmia should be classified as VT according to LC I. Part (c): a fourbeat tachyarrhythmia episode of 'de novo' VF as this ventricular tachyarrhythmia is diagnosed as VT according to LC I, while the same episode is diagnosed as VF according to LC II. The ECG examples were recorded in three isolated, Langendorff perfused rat hearts subjected to regional ischaemia for $30 \mathrm{~min}$. 
agreement was found between them when VT incidence was tested either according to LC I or according to LC II (Table 3).

Importantly, when VF incidence obtained according to LC I was tested, very strong inter-observer agreements (high kappa values) were found among the two independent investigators (Observers A and B) and the investigator of the original investigation (Observer O), (5) (Table 3). When arrhythmia definitions of LC II were applied, a good agreement was found in VF incidence between the two independent investigators; however, the kappa value was lower than that obtained according to LC I (Table 3).

The onset time of ischaemic ventricular fibrillation in the first set of experiments

When hearts were perfused with the low concentrations of the drugs and Krebs solution contained $3 \mathrm{mM} \mathrm{K}^{+}$(first set of experiments), VF incidences were high in the Control, Quinidine and Lidocaine groups of hearts irrespective of the applied VF definition (Fig. 1). This allowed us to examine the effect of the applied arrhythmia definitions on the onset time of the first episode of ischaemic VF in these three groups. Both observers found that VF onset time was significantly reduced in these three groups, when arrhythmia definitions of LC II were used as compared with the respective values obtained by applying arrhythmia definitions of LC I (Fig. $5 a$ and $5 b$ ). This shows that some ischaemic arrhythmias, which were not identified as VF according to LC I, were identified as VF according to LC II, and these arrhythmias were found not only in drug-free hearts but in quinidine and lidocaine treated hearts, too. When VT definition of LC II was applied, VT onset times were not significantly affected in any of the groups as compared with the respective values obtained by applying VT definition of LC I (data not shown).

\section{The onset time of ventricular fibrillation in all hearts that} experienced ventricular fibrillation irrespective of $K^{+}$content of the perfusate and drug treatment

When all those hearts were examined that experienced ischemic VF according to both LC I and LC II in the whole investigation, the two independent observers found that applying arrhythmia definitions of LC II significantly reduced the onset time of the first episode of VF as compared with the value obtained by using arrhythmia definitions of LC I (Fig. 5c). Applying VF definition of LC II did not increase VF onset time in any of the hearts, but reduced this variable in approximately $80 \%$ of the hearts (Table 4 ). These results did not differ qualitatively between the two independent observers (Table 4).

\section{The numbers of ventricular tachycardia and ventricular fibrillation episodes}

In order to examine whether applying arrhythmia definitions of LC II causes an 'arrhythmia shift' from the VT class to the VF class, the numbers of VT and VF episodes were calculated in the control and the flecainide treated groups of the first set of experiments ( $\mathrm{n}=12$ hearts in each group). These two groups were chosen, as the greatest and least number of arrhythmias occurred in the Control and Flecainide groups, respectively. First, a statistical analysis was performed on the data of those eight hearts of the flecainide treated group of the first set of experiments, in which neither Observer A nor Observer B found VF according to LC I, but both of them identified VF according to LC II. In the flecainide treated 8 hearts, both observers found that applying VT definition of LC II reduced the number VT episodes as compared with the respective values obtained by using VT definition of LC I (Fig. 6). On the other hand, applying VF definition of LC II significantly increased the number of VF episodes as compared with the respective zero value obtained by using VF definition of LC I (Fig. 6). Interestingly; Observer A found that arrhythmia analysis according to LC II significantly increased the cumulative number of VT and VF episodes as compared with the respective value obtained according to LC I in the flecainide treated 8 hearts. This suggests that when arrhythmia analysis was performed according to LC II, VT episodes were not only shifted to the VF class, but were also fragmented (i.e. broken up into shorter episodes due to containing diastolic pauses). Observer B did not find any significant effect of LC II on the cumulative number of VT and VF episodes (Fig. 6). Analysis of the number of the VT and VF episodes in the 12 hearts of the control group of the first set of experiments yielded qualitatively the same results despite identifying VF episodes not only by LC II but LC I, too (Fig. 7).

\section{DISCUSSION}

Present results show that arrhythmia analysis according to LC II qualitatively changed conclusions about pharmacological effects of flecainide. The change in tachyarrhythmia definitions also affected well documented pathophysiological effects, i.e. the preventive effect of the high $\mathrm{K}^{+}$concentration against ischaemic VF disappeared when the VF definition of LC II was applied. The reason behind these changes was that VF definition of LC II identified 'de novo' VF episodes not identified according to LC I, which is supported by the data showing that application of the VF definition of LC II increased VF incidence and reduced VF onset time. Importantly, a very strong interobserver agreement was found among the two independent investigators, when VF incidence obtained according to LC I was tested. Applying VF definition of LC II resulted in less interobserver agreement on VF incidence.

\section{Arrhythmia shift from ventricular tachycardia to ventricular fibrillation category enlarges the ventricular fibrillation class of LC II and markedly increases sensitivity of ventricular fibrillation detection}

VF definition of LC II diagnosed VF in all hearts, in which VF was identified according to LC I. Moreover, there were many other hearts, in which VF was detected only according to LC II and VF was not found according to LC I. Also, applying VF definition of LC II did not increase VF onset time in any of the hearts, however reduced this variable in the majority of the hearts. These results show that the VF class of LC II incorporated and enlarged the VF class defined by LC I. The reason behind this is that VF definition of LC II not only identified VF episodes detected by LC I, but also identified 'de novo' VF episodes not detected according to LC I. Our results clearly show that the enlarged VF category of LC II substantially increased the sensitivity of VF detection.

Comparing tachyarrhythmia definitions of LC I and LC II (Table 1) explains the mechanism of detection of 'de novo' VF episodes according to LC II. While arrhythmias containing 4 or more consecutive ventricular premature beats with nonprogressive variation in peak-peak interval, height and intrinsic shape should be classified as VTs according to LC I, the same arrhythmias are now shifted to the VF category of LC II (Fig. 8, Table 1) $(3,4)$. These polymorphic VTs of LC I form the 'de novo' VF episodes detected by LC II. This 'arrhythmia shift' of LC II is also indicated by the data showing that applying arrhythmia definitions of LC II reduced the number of VT episodes and simultaneously increased the number of VF episodes. 
Interestingly, Observer A found that when arrhythmia definitions of LC II were applied, the cumulative number of VT and VF episodes was greater than the respective value obtained according to LC I. The phenomenon of 'arrhythmia fragmentation' is most likely the reason behind this result. LC II introduced the concept of the importance of 'diastolic pause'. According to LC II, "if an arrhythmia is interrupted by an identifiable pause or quiescence (a 'diastolic pause', or a flat isoelectrical line) that is longer than the prevailing sinus rate (or idioventricular rate if the $\mathrm{AV}$ node is blocked) then it makes more sense to classify each arrhythmia present before and after the pause as separate events" (4). This concept enables an arrhythmia episode to be fragmented into many shorter arrhythmia episodes. Consequently, the number of VT and VF episodes measured according to LC I can be affected by 'arrhythmia fragmentation' when the same arrhythmias are evaluated according to LC II. However, the increase in VF incidence and the reduction in VF onset time caused by LC II can only be explained by the 'arrhythmia shift' phenomenon, and 'arrhythmia fragmentation' could not contribute to these results. It can be concluded that the 'arrhythmia shift' caused by LC II is responsible for the only moderate intra-observer agreement on VF incidence values obtained according to LC I and LC II.

Applying the ventricular fibrillation definition of LC II may change the conclusions of pharmacological investigations

Applying VF definition of LC II qualitatively changed conclusions about the pharmacological effect of flecainide by questioning the significant antifibrillatory effect of the drug seen when the VF definition of LC I was applied. The effectiveness of flecainide against ischaemic VF is not well characterized as results of previous investigations are confounding (10-14). Thus, it is not possible to decide which VF definition can provide data that describes the real face of this drug. However, our results provide the first clear cut evidence that by changing the definition of VF the analysis of the same ECG recordings may yield a completely different conclusion.

The analysis of the VF onset times in the first set of experiments showed that application of the VF definition of LC II increases VF detection in all groups, not only in the flecainidetreated group, but even in the quinidine- and lidocaine-treated groups and also in the control group. These results imply that the change in VF definition may retrospectively change the results of previous pharmacological studies analysed according to LC I. As mentioned earlier, over 1000 studies have already based their conclusions on data obtained according to the definitions of LC I. Changing the definition of VF may invalidate the conclusions of many of these previous investigations, and also make it impossible to compare future results that will have been obtained according to LC II to previous results obtained according to LC I.

Effect of $\mathrm{K}^{+}$on ventricular fibrillation incidence, application of the ventricular fibrillation definition of LC II may invalidate well-accepted physiological and pathophysiological concepts and studies based on these concepts

Hyperkalaemia can occur in various experimental and clinical settings (15-17). The physiological and pathophysiological effects of potassium in the development of arrhythmias have been intensively studied in earlier investigations. Arrhythmias following coronary occlusion may depend on factors such as serum potassium, i.e. higher potassium concentrations reduce the incidence of ischaemic VF in rats (18). Hyperkalaemia is antiarrhythmic during myocardial ischemia and infarction in man; serum potassium concentrations obtained on admission to hospital were inversely related to the incidence of ventricular fibrillation (19). An association between low serum potassium concentrations and ventricular arrhythmias has also been observed by a number of investigators (20) and an increased frequency of ventricular fibrillation in patients with low serum potassium concentrations was also demonstrated (21). Furthermore, in the GISSI-2 trial, the incidence of VF among patients with a serum potassium $<3.6 \mathrm{mEq} / \mathrm{L}$ was nearly twice that seen in patients with a higher serum potassium levels (22). Only one of the above mentioned investigations (18) used the VF definition of LC I, still there was a good agreement between these studies on their results about the effect of potassium on ischaemic VF. Thus, it can be concluded that the effect of potassium on ischaemic VF is independent of the VF definition of LC I. However, based on the results of the above mentioned investigations and the results of several other previous experimental and clinical investigations, it is now widely accepted that the incidence of ischaemic VF inversely correlates with the potassium concentration $(3,4,18,23)$.

When we analysed the effect of $\mathrm{K}^{+}$concentration on VF incidence in the control groups and applied the VF definition of LC I, results were in a good agreement with the above mentioned experimental and clinical data. This analysis showed that VF incidence was significantly lower in the control group perfused with Krebs solution containing $5 \mathrm{mM} \mathrm{K}^{+}$than that in the control group perfused with $3 \mathrm{mM} \mathrm{K}{ }^{+}$. However when we applied the VF definition of LC II, the VF incidence was very high in the control groups irrespective of the $\mathrm{K}^{+}$concentration of the Krebs solution. This suggests that increased sensitivity of VF detection according to LC II coincided with markedly reduced specificity. Consequently, application of VF definition of LC II substantially changed the conclusion about the physiological and pathophysiological effects of $\mathrm{K}^{+}$concentration on VF development. Present results provide the first clear-cut evidence that applying VF definition of LC II may change the conclusion not only in pharmacological but also in physiological and pathophysiological arrhythmia investigations.

Importantly, application of the VF definition of LC II would totally invalidate the whole proarrhythmia investigation with 5 $\mathrm{mM} \mathrm{K}{ }^{+}$in the perfusate in $3^{\text {rd }}$ and $4^{\text {th }}$ set of experiments in the original investigation $(5,6)$, as increased $\mathrm{VF}$ incidence in the control hearts perfused with $5 \mathrm{mM} \mathrm{K}^{+}$does not provide scope for examining profibrillatory effects of the test drugs. Present results show that application of the VF definition of LC II may invalidate a widely known and accepted concept of pathophysiology of arrhythmia development, and also invalidate earlier investigations based on this concept. This is a warning sign, which suggests that the tachyarrhythmia (VT and VF) definitions of LC II should be abandoned or at least amended in order to provide greater compatibility with the results of earlier investigations.

Excellent inter-observer agreement with ventricular fibrillation definition of LC I, smaller inter-observer agreement with ventricular fibrillation definition of LC II

Present investigation provides a strong evidence that VF results are well reproducible and the inter-observer agreement is high, when the VF definition of LC I is applied. This suggests that the VF definition of LC I is clear and it is not necessary to be changed in order to provide greater objectivity during arrhythmia evaluation, which was one of the main aims of introducing the new VF definition of LC II (4). This aim was clearly not fulfilled by the new VF definition of LC II, as applying VF definition of LC II resulted in less inter-observer agreement on VF incidence. Furthermore, VT definition of LC II did not remarkably improve inter-observer agreement on VT incidence. These suggest that applying VT and VF definitions of 
LC II does not improve compatibility of the results of various research groups and investigators, which further emphasizes the need for an amendment of the VT and VF definitions of LC II.

\section{Conclusions}

Present investigation provides the first clear-cut evidence that arrhythmia results obtained by applying ventricular tachyarrhythmia definitions of LC I and LC II are not compatible in Langendorff perfused rat hearts. This calls for further research in order to test the validity of the arrhythmia definitions of LC II in other species and models, too. Nevertheless, present results indicate that applying VF definition of LC II may change the conclusion of previous pharmacological, physiological and pathophysiological arrhythmia investigations. The reason behind this is that VF class of LC II incorporated and enlarged the VF class defined by LC I. The enlarged VF category of LC II substantially increases the sensitivity of VF detection, but it coincides with markedly reduced specificity. Furthermore, present investigation provides an evidence that VF incidence results are well reproducible and the inter-observer agreement is high, when the VF definition of LC I is applied. However, applying VF definition of LC II may reduce inter-observer agreement on VF incidence. Thus, it is concluded that VT and VF definitions of LC II should be amended in order to allow better inter-observer agreement and greater compatibility with the results of earlier investigations obtained by applying arrhythmia definitions of LC I. However, any modified definitions should be subjected to research in order to test their validity.

Assaf Regev and Hedvig Takacs equally contributed and share first authorship.

Acknowledgements: Dr. Michael J. Curtis is thanked for designing and supervising the Langendorff rat heart experiments performed in his laboratory at the Cardiovascular Division, King's College London, London, UK.

This work was supported by Hungarian Scientific Research Fund [OTKA PD 105882], the UNKP-17- 4 New National Excellence Program of The Ministry of Human Capacities, and EFOP-3.6.2-16-2017-00006, EFOP-3.6.1-16-2016-00008.

Conflict of interests: None declared.

\section{REFERENCES}

1. Priori SG, Blomstrom-Lundqvist C, Mazzanti A, et al. 2015 ESC Guidelines for the management of patients with ventricular arrhythmias and the prevention of sudden cardiac death: The Task Force for the Management of Patients with Ventricular Arrhythmias and the Prevention of Sudden Cardiac Death of the European Society of Cardiology (ESC) Endorsed by: Association for European Paediatric and Congenital Cardiology (AEPC). Europace 2015; 17: 1601-1687.

2. Al-Khatib SM, Stevenson WG, Ackerman MJ, et al. 2017 AHA/ACC/HRS Guideline for management of patients with ventricular arrhythmias and the prevention of sudden cardiac death: executive summary: a report of the American College of Cardiology/American Heart Association Task Force on Clinical Practice Guidelines and the Heart Rhythm Society. Heart Rhythm 2018; 15: e190-e252. doi: 10.1016/ j.hrthm.2017.10.035

3. Walker MJ, Curtis MJ, Hearse DJ, et al. The Lambeth Conventions: guidelines for the study of arrhythmias in ischaemia infarction, and reperfusion. Cardiovasc Res 1988; 22: 447-455.
4. Curtis MJ, Hancox JC, Farkas A, et al. The Lambeth Conventions (II): guidelines for the study of animal and human ventricular and supraventricular arrhythmias. Pharmacol Ther 2013; 139: 213-248.

5. Farkas A, Curtis MJ. Limited antifibrillatory effectiveness of clinically relevant concentrations of class I antiarrhythmics in isolated perfused rat hearts. $J$ Cardiovasc Pharmacol 2002; 39: 412-424.

6. Farkas A, Curtis MJ. Does QT widening in the Langendorffperfused rat heart represent the effect of repolarization delay or conduction slowing? J Cardiovasc Pharmacol 2003; 42: 612-621.

7. Sarusi A, Rarosi F, Szucs M, et al. Absolute beat-to-beat variability and instability parameters of ECG intervals: biomarkers for predicting ischaemia-induced ventricular fibrillation. Br J Pharmacol 2014; 171: 1772-1782.

8. Altman DG. Practical Statistics for Medical Research. London: Chapman \& Hall; 1991.

9. Cohen J. A coefficient of agreement for nominal scales. Educ Psychol Meas 1960; 20: 37-46.

10. Gout B, Nichols AJ, Feuerstein GZ, Bril A. Antifibrillatory effects of BRL-32872 in anesthetized Yucatan minipigs with regional myocardial ischemia. $J$ Cardiovasc Pharmacol 1995; 26: 636-644.

11. Lederman SN, Wenger TL, Bolster DE, Strauss HC. Effects of flecainide on occlusion and reperfusion arrhythmias in dogs. J Cardiovasc Pharmacol 1989; 13: 541-546.

12. Winslow E, Campbell JK, Barron E, Marshall RJ, Muir AW. Effects of Org 7797 on early, late and inducible arrhythmias following coronary artery occlusion in rats and dogs. $\mathrm{Br} J$ Pharmacol 1991; 104: 853-858.

13. Barrett TD, Hayes ES, Walker MJ. Lack of selectivity for ventricular and ischaemic tissue limits the antiarrhythmic actions of lidocaine, quinidine and flecainide against ischaemia-induced arrhythmias. Eur J Pharmacol 1995; 285: 229-238.

14. Barrett TD, Hayes ES, Yong SL, Zolotoy AB, Abraham S, Walker MJ. Ischaemia selectivity confers efficacy for suppression of ischaemia-induced arrhythmias in rats. Eur $J$ Pharmacol 2000; 398: 365-374.

15. Deska P, Nowicki M. Short-term changes of serum potassium concentration induced by physical exercise in patient with arterial hypertension treated with angiotensinconverting enzyme inhibitor alone or in combination with statin. J Physiol Pharmacol 2017; 68: 133-138.

16. Wojcik B, Knapp M, Gorski J. Non-ischemic heart preconditioning. J Physiol Pharmacol 2018; 69: 173-184.

17. Wojcik B, Miklosz A, Zabielski P, Chabowski A, Gorski J. Effect of tachycardia on mRNA and protein expression of the principal components of the lipolytic system in the rat's heart ventricles. J Physiol Pharmacol 2017; 68: 731-736.

18. Curtis MJ, Hearse DJ. Ischaemia-induced and reperfusioninduced arrhythmias differ in their sensitivity to potassium: implications for mechanisms of initiation and maintenance of ventricular fibrillation. J Mol Cell Cardiol 1989; 21: 21-40.

19. Nordrehaug JE, von der Lippe G. Hypokalaemia and ventricular fibrillation in acute myocardial infarction. $\mathrm{Br}$ Heart J 1983; 50: 525-529.

20. Papp H, Sarusi A, Farkas AS, et al. Hyperventilation assists proarrhythmia development during delayed repolarization in clofilium-treated, anaesthetized, mechanically ventilated rabbits. J Physiol Pharmacol 2016; 67: 731-737.

21. Solomon RJ. Ventricular arrhythmias in patients with myocardial infarction and ischaemia. The role of serum potassium. Drugs 1986; 31 (Suppl 4): 112-120.

22. Volpi A, Cavalli A, Santoro L, Negri E. Incidence and prognosis of early primary ventricular fibrillation in acute 
myocardial infarction--results of the Gruppo Italiano per lo Studio della Sopravvivenza nell'Infarto Miocardico (GISSI-

2) database. Am J Cardiol 1998; 82: 265-271.

23. Cohn JN, Kowey PR, Whelton PK, Prisant LM. New guidelines for potassium replacement in clinical practice: a contemporary review by the National Council on Potassium in Clinical Practice. Arch Intern Med 2000; 160: 2429-2436.

Received: October 17, 2018

Accepted: February 28, 2019

Author's address: Dr. Andras Farkas, Second Department of Medicine and Cardiology Centre, Faculty of Medicine, University of Szeged, 8 Semmelweis Street, H-6725 Szeged, Hungary.

E-mail: farkas.andras@med.u-szeged.hu 